\title{
Investigating 3,4-bis(3-nitrofurazan-4-yl)furoxan Detonation with a Rapidly Tuned Density Functional Tight Binding Model
}

\author{
Rebecca K. Lindsey, ${ }^{1, \text { a) }}$ Sorin Bastea, ${ }^{1}$ Nir Goldman, ${ }^{1,2}$ and Laurence E. Fried ${ }^{1}$ \\ 1) Physical and Life Sciences Directorate, Lawrence Livermore National Laboratory, \\ Livermore, California 94550, United States \\ 2) Department of Chemical Engineering, University of California, Davis,
} California 95616, United States

(Dated: 16 February 2021)

We describe a machine learning approach to rapidly tune density functional tight binding models for description of detonation chemistry in organic molecular materials. Resulting models enable simulations on the several 10s of ps scales characteristic to these processes, with "quantum-accuracy." We use this approach to investigate early shock chemistry in 3,4-bis(3-nitrofurazan-4-yl)furoxan, a hydrogen-free energetic material known to form onion-like nanocarbon particulates following detonation. We find that the ensuing chemistry is significantly characterized by formation of large $\mathrm{C}_{x} \mathrm{~N}_{y} \mathrm{O}_{z}$ species, which are likely precursors to the experimentally observed carbon condensates. Beyond utility as a means of investigating detonation chemistry, the present approach can be used to generate quantum-based reference data for development of full machine-learned interatomic potentials capable of simulation on even greater time and length scales, i.e. for applications where characteristics timescales exceed the reach of methods including Kohn-Sham density functional theory which are commonly used for reference data generation.

a) To whom correspondence should be addressed: lindsey11@llnl.gov 


\section{INTRODUCTION}

Evolution of organic molecular materials under extreme conditions (e.g. 1000s of K and 10 s of $\mathrm{GPa}$ ) is central to research spanning from astrochemistry ${ }^{1-3}$ to nanomaterial fabrication $^{4-6}$. However, a confluence of challenges including complicated physical and chemical processes exhibiting a broad range of characteristic time and length scales have made a clear picture elusive $e^{4,5,7-11}$. For example, materials lacking sufficient oxygen to undergo complete combustion are known to form carbon condensates (e.g. nanodiamond, graphitic nano-onions and nano-ribbons, etc.) when driven to high temperature and pressure (e.g. via shockwaves), where resulting condensate size, composition, and allotrope are governed by the starting material's chemical composition, the thermodynamic pathway through which it is heated, compressed, and eventually expanded and cooled, and the associated chemistry and kinetics ${ }^{4}$. Ultimately, this process involves phenomena occurring on both nanometer length scales over 10s of picoseconds (e.g. chemistry mediated condensate formation), as well as those occurring on scales approaching microns and microseconds (e.g. condensate growth and aggregation $)^{6,12-14}$. Material evolution under extreme conditions is often studied through detonation or shock experiments, where the resulting dynamic and transient conditions can make determination of fundamental properties like system temperature exceedingly challenging and ultimately preclude elucidating early time chemistry driving carbon cluster formation.

Atomistic simulations excel at probing sub-micron and -microsecond scales and can provide complementary insights (e.g. determining phase boundaries, ${ }^{15}$ assigning temperature for shock experiments ${ }^{16,17}$, investigating thermal decomposition ${ }^{18}$, etc.); however, this approach hinges upon the underlying interatomic model. Quantum mechanical methods including Kohn-Sham density functional theory ${ }^{19}$ (DFT) have been shown to be well suited for studying materials under extreme conditions ${ }^{17,20,21}$, but high associated computational cost precludes access to timescales relevant to shockwave-driven chemistry (e.g. several 10s of picoseconds to microseconds). Molecular mechanics-based simulations can overcome this difficulty and enable access to much larger time and length scales; however, models capable of accurately describing system structure, dynamics, speciation, and kinetics under extreme conditions are scarce, and generating new parameter sets remains a significant undertak-

ing. Density functional theory tight binding with self-consistent-charges ${ }^{22}$ (DFTB-SCC) is a 


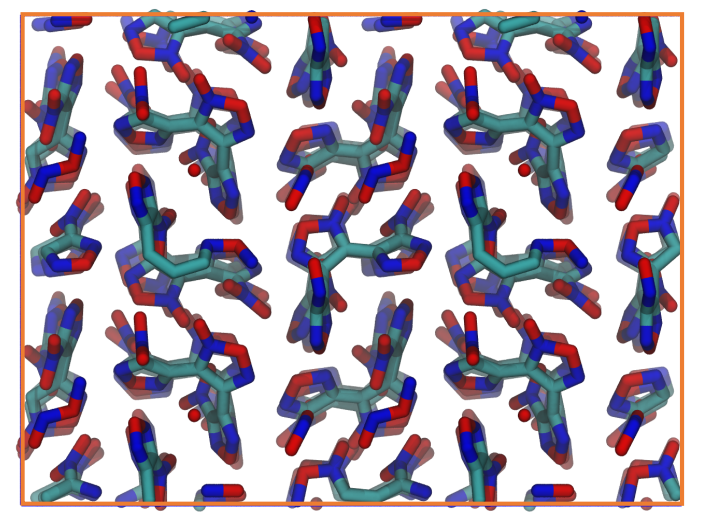

FIG. 1. Snapshot of a thermalized DNTF crystal. Atoms in cyan, blue, and red correspond to carbon, nitrogen, and oxygen, respectively.

practical alternative, exhibiting substantially improved predictive power over molecular mechanic approaches, and a 3-orders-of-magnitude increase in simulation efficiency over DFT. However, this approximate quantum-mechanical method utilizes parameter sets that are not generally designed for application to organic molecular materials under extreme conditions.

In this paper, we show how machine learning (ML) can be used to generate corrections to standard DFTB models enabling quantum-accurate simulations for shock compressed materials on timescales substantially greater than those accessible to DFT. We focus here on developing a model for the energetic material 3,4-bis(3-nitrofurazan-4-yl)furoxan (i.e. $\mathrm{C}_{6} \mathrm{~N}_{8} \mathrm{O}_{8}$, often referred to as DNTF or BNFF), shown in figure 1. DNTF exhibits an usually high detonation temperature and has been recently shown to produce liquid carbon nanodroplets upon detonation, which subsequently solidify into graphitic nano-onions ${ }^{6}$. Using the developed model, we investigate early decomposition chemistry under conditions of pressure and temperature typical of strong shocks. Results are compared with predictions from thermochemical calculations, and are discussed in the context of studies probing the relationship between chemical kinetics, thermodynamics, oxygen balance and carbon condensation in shock compressed organic molecular materials. Specifically, we aim to shed light on: (1) what are the major chemical kinetics steps associated with shock decomposition of DNTF? (2) what is the equation of state of the shocked material? (3) how do these results compare with those for other carbon-rich compounds? and (4) what are the implications of the present results for nanocarbon formation mechanisms in DNTF and in general? In the following sections we provide a high-level overview of DFTB-SCC, describe 
the ML approach used to generate a model tuned for DNTF, and validate it by comparing model-predicted DNTF structure, equation of state, speciation, and kinetics with that from DFT. Following, speciation, kinetics, and the shock Hugoniot equation of state are explored and compared with existing thermochemical models. Ultimately, we find that our ML-tuned DFTB approach is well suited for the study of organic molecular materials subject to extreme temperature and pressure conditions and can provide important insights into the early chemistry and kinetics.

\section{COMPUTATIONAL METHODS}

All DFT simulations were performed for systems of 264 atoms using the VASP ${ }^{23-26}$ package with a planewave cutoff of $1000 \mathrm{eV}$, the Perdew-Burke-Ernzerhof generalized gradient approximation functional $^{27,28}$ (PBE), projector-augmented wave pseudopotentials ${ }^{29,30}$ (PAW), the DFT-D2 method $^{31}$ for description of dispersion interactions, and a 0.5 fs time-step. Simulations in the canonical (i.e. $N V T$ ) ensemble used a Nose-Hoover thermostat ${ }^{32,33}$. DNTF density at the melting point was approximated through a 6 ps isobaric-isothermal (i.e. $N p T$ ) ensemble simulation using Langevin dynamics with a Parrinello-Rahman barostat ${ }^{34,35}$.

DFTB forces, stresses, and energies were evaluated with self-consistent charges (SCC), using the DFTB + package $^{36}$ with the the Mio-1-1 parameter set (available at http://www.dftb.org), unless otherwise stated. The SCC convergence criteria was set to $10^{-6}$ au and the Universal Force Field ${ }^{37}$ (UFF) was used as a dispersion correction. A timestep of 0.5 fs was used for systems at or below the DNTF melting point and a value of 0.2 fs was used otherwise. DFTB calculations in the canonical ensemble were performed with a Nose-Hoover thermostat, for systems of 264 atoms, while shock simulations were run for 176-atom systems. DFTB-ChIMES model simulation results are presented as averages across eight independent simulations. A locally modified version of DFTB + was used for ML-corrected DFTB calculations; a library for computing ChIMES interactions, which also contains all published parameter sets, is available at https://github.com/rk-lindsey/chimes_calculator. We note that simulations using the Mio+ChIMES model are of approximately the same efficiency as those using Mio-1-1 alone (i.e. ChIMES evaluations take $<0.1 \mathrm{~s} /$ ionic-step compared to $\approx 13 \mathrm{~s} /$ ionic-step for Mio-1-1 alone), and that in general, the present DFTB simulations provide a $>2.5 \times 10^{3}$ increase in steps/CPU-hr relative to DFT. 


\section{A. DFTB Overview}

The DFTB-SCC formalism has been discussed in detail elsewhere $22,36,38,39$. Briefly, the method assumes neutral, spherically symmetric charge densities on the atoms and expands the DFT Hamiltonian to second-order in charge fluctuations. The DFTB total energy is expressed as

$$
E_{\mathrm{DFTB}}=E_{\mathrm{BS}}+E_{\mathrm{Coul}}+E_{\mathrm{disp}}+E_{\mathrm{rep}}
$$

where $E_{\mathrm{BS}}$ corresponds to the band structure energy, $E_{\text {Coul }}$ is a self-consistently computed charge fluctuation term, $E_{\text {disp }}$ is an empirical dispersion correction, and $E_{\text {rep }}$ is an empirical term accounting for ion-ion repulsions and Hartree and exchange-correlation double counting terms. The computational efficiency of DFTB is primarily realized through $E_{\mathrm{BS}}$, which is taken as a sum over occupied electronic states from the approximate DFTB Hamiltonian. $E_{\mathrm{BS}}$ is usually computed from pre-tabulated Slater-Koster tables derived from DFT calculations with a minimal basis set, giving rise to an orders-of-magnitude increase in computational efficiency for a given system size, relative to DFT. Numerous DFTB Slater-Koster and $E_{\text {rep }}$ parameter sets are available (i.e. at http://www.dftb.org), but these models are typically intended for biological systems (i.e. ambient $T$ and $p$ ) or inorganic materials. Below, we will describe how the Chebyshev Interaction model for Efficient Simulation (ChIMES) can be used to introduce a correction term $\left(E_{\text {corr }}\right)$ to an existing DFTB model (Mio-1-1) for description of DNTF under extreme $T$ and $p$. We note that, for the remainder of this work, the resulting model will be referred to as Mio + ChIMES.

\section{B. ChIMES Overview}

ChIMES $^{40-44}$ is a machine learned (ML) reactive interatomic interaction potential (IAP) explicitly describing many-body interactions through linear combinations of Chebyshev polynomials. Though ChIMES has been applied as a standalone IAP to systems spanning ambient water ${ }^{42}$ to molten carbon ${ }^{40}$ and shocked carbon monoxide ${ }^{9}$, it has only been used as a DFTB correction for description of non-organic materials ${ }^{41}$, to date. The ChIMES total energy is given by

$$
E_{n_{\mathrm{B}}}=\sum_{i_{1}}^{n_{\mathrm{a}}}{ }^{1} E_{i_{1}}+\sum_{i_{1}>i_{2}}^{n_{\mathrm{a}}}{ }^{2} E_{i_{1} i_{2}}+\sum_{i_{1}>i_{2}>i_{3}}^{n_{\mathrm{a}}}{ }^{3} E_{i_{1} i_{2} i_{3}}+\cdots+\sum_{i_{1}>i_{2} \ldots}^{n_{\mathrm{a}}} \sum_{i_{n_{\mathrm{B}}-1}>i_{n_{\mathrm{B}}}}^{n_{\mathrm{B}}} E_{i_{1} i_{2} \ldots i_{n_{\mathrm{B}}}},
$$


where $E_{n_{\mathrm{B}}}$ is the total ChIMES system energy, $n_{\mathrm{B}}$ is the maximum bodiedness, ${ }^{n} E_{i_{1} i_{2} \ldots i_{n}}$ in is the $n$-body ChIMES energy for a given set of $n$ atoms with indices $i=i_{1}, i_{2}, \ldots i_{n}$, and $n_{a}$ is the total number of atoms in the system. We note that ChIMES takes the single body interactions as constant values. ChIMES models developed in the present work include 1-through-3-body interactions, with 2-body interactions are given by

$$
{ }^{2} E_{i j}=f_{\mathrm{p}}\left(r_{i j}\right)+f_{\mathrm{s}}^{e_{i} e_{j}}\left(r_{i j}\right) \sum_{n=1}^{\mathcal{O}_{2 \mathrm{~B}}} c_{n}^{e_{i} e_{j}} T_{n}\left(s_{i j}^{e_{i} e_{j}}\right),
$$

where $f_{\mathrm{p}}$ is a penalty function which discourages sampling of unphysically short pair distances, $r_{i j}$ is the distance between two atoms $i$ and $j$. The function $f_{\mathrm{s}}^{e_{i} e_{j}}\left(r_{i j}\right)$ ensures the potential energy surface for interactions between atoms $i$ and $j$, (i.e. which are of element types $e_{i}$ and $e_{j}$, respectively) decays smoothly to zero at the the outer cutoff. The sum over $c_{n}^{e_{i} e_{j}} T_{n}\left(s_{i j}^{e_{i} e_{j}}\right)$ gives a polynomial series of order $\mathcal{O}_{2 \mathrm{~B}}$, where $T_{n}$ is an order $n$ Chebyshev polynomial of the first kind, $c_{n}^{e_{i} e_{j}}$ is the corresponding coefficient, and the argument $s_{i j}^{e_{i} e_{j}}$ gives a pair distance transformed to span the [-1,1] domain over which Chebyshev polynomials are defined. For a detailed description of $f_{\mathrm{p}}^{e_{i} e_{j}}, f_{\mathrm{s}}^{e_{i} e_{j}}$, and transformation of $r_{i j}$ to $s_{i j}^{e_{i} e_{j}}$, the reader is referred to the supporting information and 44 .

The ChIMES 3-body energy between atoms $i, j, k$ is constructed from interaction terms for the constituent atom pairs $i j, i k, j k$, i.e.:

$$
E_{i j k}=f_{\mathrm{s}}^{e_{i} e_{j}}\left(r_{i j}\right) f_{\mathrm{s}}^{e_{i} e_{k}}\left(r_{i k}\right) f_{\mathrm{s}}^{e_{j} e_{k}}\left(r_{j k}\right) \times \sum_{m=0}^{\mathcal{O}_{3 \mathrm{~B}}} \sum_{\substack{\mathcal{O}_{3 \mathrm{~B}} \\ \mathcal{O}_{3}}}^{\mathcal{O}_{3 \mathrm{~B}}} \sum_{q=0}^{e_{i} e_{j}, e_{i} e_{k}, e_{j} e_{k}} T_{m}\left(s_{i j}^{e_{i} e_{j}}\right) T_{p}\left(s_{i k}^{e_{i} e_{k}}\right) T_{q}\left(s_{j k}^{e_{j} e_{k}}\right),
$$

where $\mathcal{O}_{n}^{*}$ is the maximum polynomial order for an $n$ body interactions, and the asterisk indicates a sufficient number of non-zero terms exist that the graph formed by the edges of interacting atoms connects all $n$ atoms, which guarantees a true $n$-body interaction.

ChIMES coefficients were fit using the following objective function

$$
F_{\mathrm{obj}}=\frac{1}{n_{\mathrm{f}}\left(3 n_{\mathrm{a}}+n_{\mathrm{t}}+1\right)} \times \sum_{i=1}^{n_{\mathrm{f}}}\left[\sum_{j=1}^{n_{\mathrm{a}}} \sum_{k=1}^{3} w_{\mathrm{F}_{i j k}}^{2}\left(\Delta F_{i j k}\right)^{2}+\sum_{l=1}^{n_{\mathrm{t}}} w_{\sigma_{i l}}^{2}\left(\Delta \sigma_{i l}\right)^{2}+w_{\mathrm{E}_{i}}^{2}\left(\Delta E_{i}\right)^{2}\right],
$$

where $\Delta X=X^{\text {DFT }}-\left(X^{\text {DFTB,BS }}+X^{\text {DFTB,Coul }}+X^{\text {DFTB,disp }}+X^{\text {ChIMES }\{c\}}\right) . F_{\text {obj }}$ and $\{c\}$ are the weighted root-mean-squared error and model coefficients, respectively. The number of frames and atoms are given by $n_{\mathrm{f}}$ and $n_{\mathrm{a}}$, respectively, and the factor of 1 in the denominator arises from inclusion of a single per-configuration energy, $E_{i}$. $F_{i j k}$ indicates the $k^{\text {th }}$ Cartesian component of the force on atom $j$ in configuration $i$. Units of kcal mol $\AA^{-1}$ 
and $\mathrm{kcal} \mathrm{mol}^{-1}$ were used for forces, energies, and stresses respectively. The superscripts "ChIMES," "DFTB," and "DFT" indicate forces, energies, or stresses predicted from the present ChIMES correction, DFTB and the DFT molecular dynamics (DFT-MD) training trajectory, respectively.

Because ChIMES models are parameterically linear, coefficients can be rapidly obtained by solving the following overdetermined matrix equation:

$$
\boldsymbol{w} \boldsymbol{M c}=\boldsymbol{w} \boldsymbol{X}^{\mathrm{DFT}}
$$

where $\boldsymbol{X}^{\mathrm{DFT}}$ is the vector of $F_{i j k}^{\mathrm{DFT}}, E_{i}$ and $\sigma_{i l}^{\mathrm{DFT}}$ values, $\boldsymbol{w}$ is a diagonal matrix of weights to be applied to the elements of $\boldsymbol{X}^{\mathrm{DFT}}$ and rows of $\boldsymbol{M}$, and the elements of design matrix $\boldsymbol{M}$ are given by:

$$
M_{a b}=\frac{\partial\left(X^{\mathrm{DFTB}, \mathrm{BS}}+X^{\mathrm{DFTB}, \mathrm{Coul}}+X^{\mathrm{DFTB}, \mathrm{disp}}+X_{a}^{\mathrm{ChIMES}\{c\}}\right)}{\partial c_{b}},
$$

where $a$ represents a combined index over force and energy components, and $b$ is the index over permutationally invariant model parameters. As is described elsewhere ${ }^{44}$, model parameters are obtained via least absolute shrinkage and selection operator ${ }^{45}$ (LASSO) regression, which optimizes a modified objective function (e.g. $\left.F_{\mathrm{LASSO}}=F_{\mathrm{obj}}+2 \lambda \sum\left|c_{i}\right|\right)$ in which the absolute values (L1 norm) have the effect of setting certain ChIMES parameter values, $c_{i}$, to 0 , and can thus lead to substantial gains in model efficiency. For this work, a regularization parameter of $\lambda=0.01$ was used and the design matrix was left un-normalized.

\section{Initial Training Set Generation}

Because our target is a model capable of describing chemistry in shock-compressed DNTF, it is critical that the training set contains data at relevant thermodynamic conditions (e.g. including detonation). However, identifying these states is difficult since the existing experimental data are very limited and previous simulation studies are lacking for this material. To overcome this challenge, thermochemical calculations ${ }^{46}$ were used to predict the reacted shock Hugoniot of DNTF up to approximately $40 \mathrm{GPa}$, encompassing the range of conditions under which recent experiments studied and characterized the carbon condensation processes $^{6}$. Three state points for generation of DFT-MD training data were identified 
TABLE I. Thermodynamic state points for DFT simulations and thermochemical Hugoniot EOS predictions. $t_{\text {sim,DFT }}$ is the total DFT simulation length. $X_{\text {full,EOS }}$ are properties (e.g. temperature, $T$, or pressure, $p$ ) predicted by thermochemical calculations for the fully reacted DNTF systems, where in this context, fully reacted corresponds to chemically equilibrated mixtures of small molecular species and carbon particles.

\begin{tabular}{|c|c|c|c|c|c|}
\hline$\rho$ & $T_{\mathrm{DFT}}$ & $P_{\mathrm{DFT}}$ & $t_{\mathrm{sim}, \mathrm{DFT}}$ & $T_{\text {full,EOS }}$ & $P_{\text {full,EOS }}$ \\
\hline $\mathrm{g} \mathrm{cm}^{-3}$ & K & $\mathrm{GPa}$ & ps & K & $\mathrm{GPa}$ \\
\hline 1.86 & 300 & 0.03 & 5.00 & 4220 & 19.7 \\
\hline 1.80 & 383 & 0.31 & 5.00 & & \\
\hline 2.00 & 2000 & 5.50 & 5.00 & & \\
\hline 2.00 & 4250 & 13.6 & 2.50 & 4250 & 20.7 \\
\hline 2.25 & 4440 & 21.0 & 2.50 & 4440 & 28.3 \\
\hline 2.50 & 4700 & 28.5 & 2.50 & 4700 & 39.0 \\
\hline 2.50 & 9000 & 35.6 & 0.25 & & \\
\hline
\end{tabular}

from this Hugoniot; three additional state points were utilized for training data generation, corresponding to the material under ambient conditions, at its melting point, and above its thermal decomposition temperature ${ }^{47}$. Finally, a high temperature training point was added to ensure adequate sampling of close interatomic contacts. These state points along with the DFT-MD simulation length are given in Table I. All simulations were initialized with the material in its molecular crystal state. We note that the DFT trajectories were fairly short and are therefore unlikely to have reached the fully chemically equilibrated state assumed by thermochemical calculations. However, the following section describes how a single iterative fitting step can significantly extend the effective timescale spanned by the training set.

\section{RESULTS}

In the following section, we discuss development of the Mio + ChIMES model and compare its performance relative to standard DFTB (i.e. Mio-1-1) in terms of recovery of DFT 

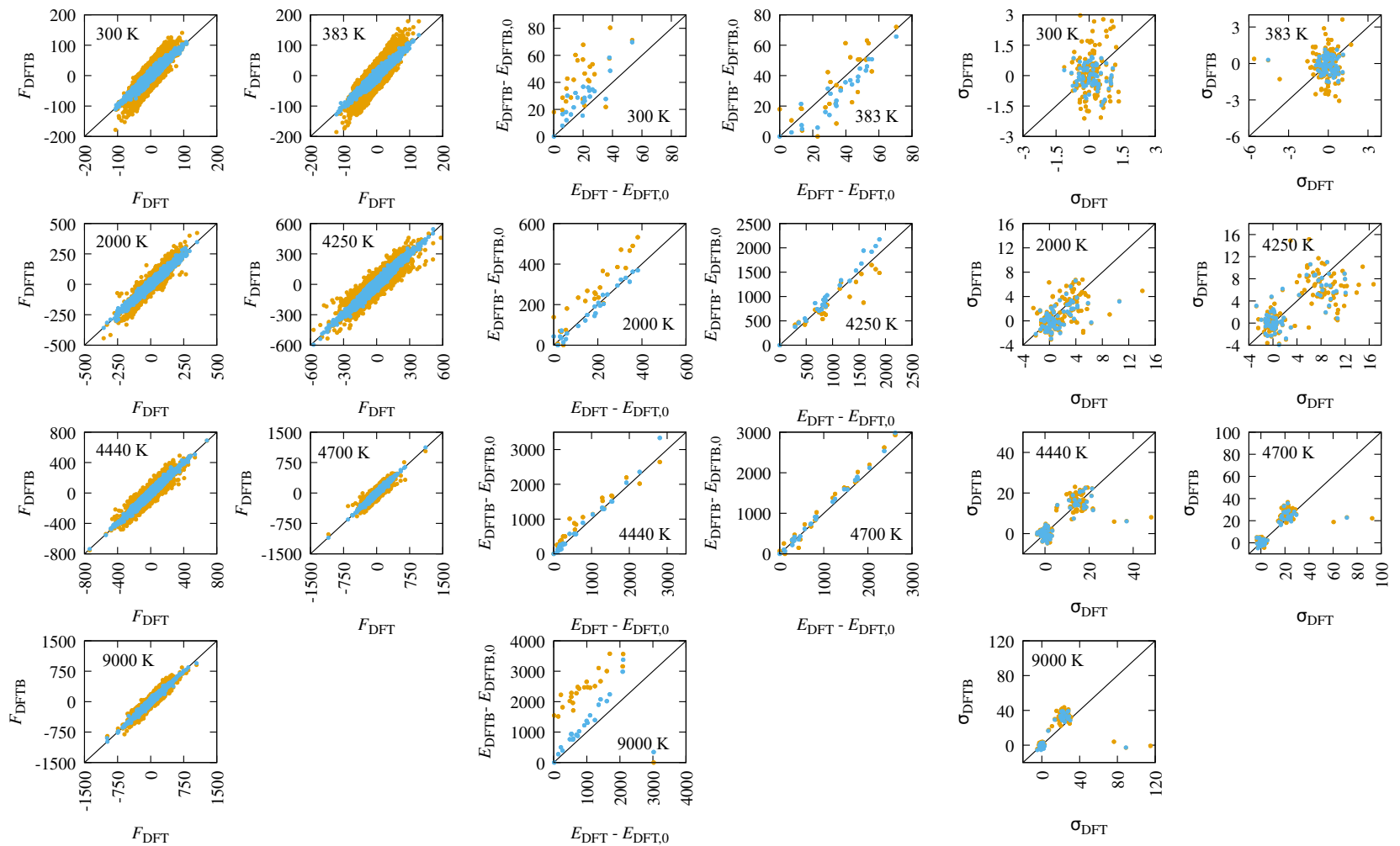

FIG. 2. Atom force components, frame energies, and frame diagonal stress tensor components predicted by Mio-1-1 (orange) and Mio+ChIMES (blue) relative to DFT, at the DFT thermodynamic state points indicated in Table I. The solid line is drawn as a guide to the eye and provides $x=y$. Forces, energies, and stresses are given in units of $\mathrm{kcal} \mathrm{mol}^{-1} \AA^{-1}, \mathrm{kcal} \mathrm{mol}^{-1}$, and GPa, respectively, and the thermodynamic state point is given as a temperature in each plot and corresponds to a training state point.

forces, energies, and stresses, and for prediction of physical properties (i.e. pressures, system structure, speciation, and kinetics). The validated model is then used to investigate DNTF decomposition kinetics, generate a "quantum-based" prediction of the material shock Hugoniot, and to explore shockwave-driven chemistry; results are discussed in relation to other carbon-rich organic materials and thermochemical calculations.

\section{A. Model Generation and Validation}

The model developed herein used polynomial orders of $\mathcal{O}_{2 \mathrm{~B}} / \mathcal{O}_{3 \mathrm{~B}}=12 / 7$, which were found to afford a balance between model complexity and accuracy. Unless otherwise spec- 
ified, model hyperparameters (e.g. inner and outer interaction cutoffs, pair Morse-like parameters, etc.) were set using previously described protocols ${ }^{42,43}$ and are given along with the remaining model parameters (e.g. Chebyshev polynomial coefficients) in the supporting information. An initial model was trained to a total of 175 configurations (i.e. 25 at each training state point). The ChIMES interaction for this system was found to be more susceptible to overfitting than standalone ChIMES models because only a very short-ranged portion of the underlying DFTB interaction is being corrected. This can prove problematic when DFT and the base DFTB model yield even small differences in longer-ranged (e.g. dispersion) interactions, since the relatively short-ranged ChIMES model may take on an unphysical form to try to account for these differences. We note that this tendency can be mitigated through weighting. For this work forces, diagonal stress tensor components, and overall system energies were considered in our fits and were assigned fitting weights of $1.0,20 e^{-5 \sigma}$, and $0.55 e^{\left(E_{\min , \mathrm{DFT}}-E\right) / 2000}$, respectively, where forces, energies, and stresses are in units of $\mathrm{kcal} \mathrm{mol}^{-1} \AA^{-1}$, $\mathrm{kcal} \mathrm{mol}^{-1}$, and GPa, respectively. The resulting model exhibited excellent recovery of DFT structure, equation of state, and chemical kinetics and was stable when extended out to 10 ps. However, exploratory shock simulations using the MSST technique ${ }^{48}$ indicated that systems sample densities significantly greater than those in the DFT training set (e.g. 3.5-4.0 $\mathrm{g} \mathrm{cm}^{-3}$ ), before reaction-driven pressure relaxation to the system's nominal reacted Hugoniot state. As a consequence, these simulations rapidly became unstable (i.e. substantial deviation in the conserved quantity was observed) due to sampling of these high density configurations which the initial model was unable to describe. The model was refit to remedy this behavior. Rather than generating training configurations at these problematic high density state points from expensive DFT-MD simulations, a single iterative refinement was performed, where approximately 50 configurations from the Mio+ChIMES MSST simulations were sent to DFT for single point evaluation, and combined with the training set from which a new model was generated. This model was found to be stable for the duration of the simulations, and as an added benefit, introduced configurations corresponding to substantially longer simulations (e.g. 10s of picoseconds) than those originally generated via DFT-MD. Figure 2 provides a comparison between forces, energies, and diagonal stress tensor components predicted by DFT, standard (Mio-1-1) DFTB, and Mio+ChIMES. Overall Mio-1-1 performance is good, but we find (1) systematic over-prediction in force magnitudes for $T=300-4250$ and larger residuals com- 


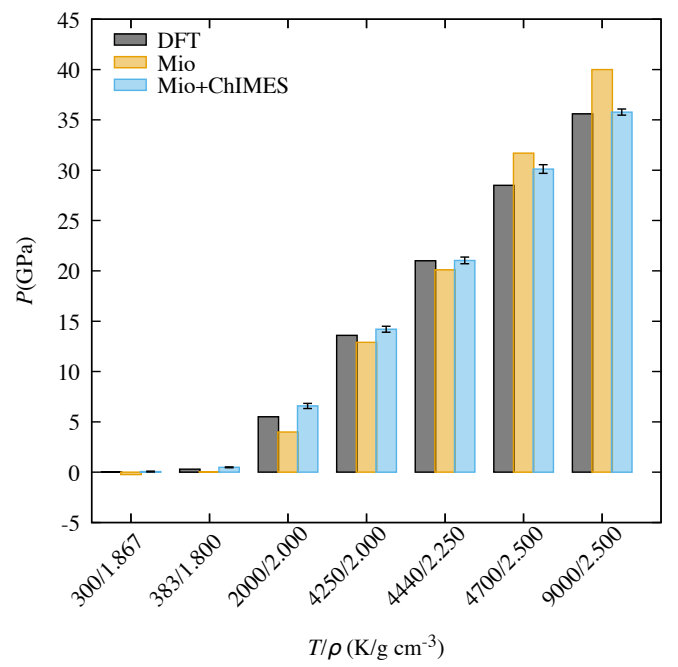

FIG. 3. DNTF pressures predicted by DFT, standard DFTB (Mio), and the DFTB-ChIMES model $($ Mio + ChIMES) at thermodynamic states spanning ambient to shock compressed conditions. Error bars provide standard deviations across independent simulations.

pared to Mio+ChIMES, (2) offsets in energies relative to DFT, particularly at 300, 4250, and $9000 \mathrm{~K}$, and (3) larger stress tensor magnitudes at 300 and $383 \mathrm{~K}$.

Performance of Mio+ChIMES relative to DFT and Mio-1-1 was also benchmarked in terms of physical properties arising from MD simulations at each of the DFT state points and length indicated in Table I. Unsurprisingly, figure 3 shows that pressure recovery at each state point is improved by the Mio + ChIMES model. The radial pair distribution functions arising from these simulations are provided in figure S1 of the supplementary information and show that, in general, Mio-1-1 and Mio + ChIMES are in equivalently excellent agreement with DFT at ambient and moderately extreme conditions (e.g. $2000 \mathrm{~K}$ and below), but the ChIMES-corrected model is found to yield significantly improved recovery of DFT structure under extreme conditions (i.e. $T>4440 \mathrm{~K}, \rho>2.25 \mathrm{~g} \mathrm{~cm}^{3}$ ).

Model performance was also investigated in terms of speciation. Figure 4 compares evolution of instantaneous bond-type counts between the DFT, Mio-1-1, and Mio+ChIMES. For this analysis, two atoms were considered bonded if their distance fell below 1.90, 1.80, $1.80,1.75,1.65$, or $1.70 \AA$ for $\mathrm{C}-\mathrm{C}, \mathrm{C}-\mathrm{N}, \mathrm{C}-\mathrm{O}, \mathrm{N}-\mathrm{N}, \mathrm{N}-\mathrm{O}$, and $\mathrm{O}-\mathrm{O}$ atom pairs, respectively. Of course, resulting bond counts will vary depending on choice of criteria; however, for the present analysis we simply aim to establish a consistent metric to compare chemistry across 

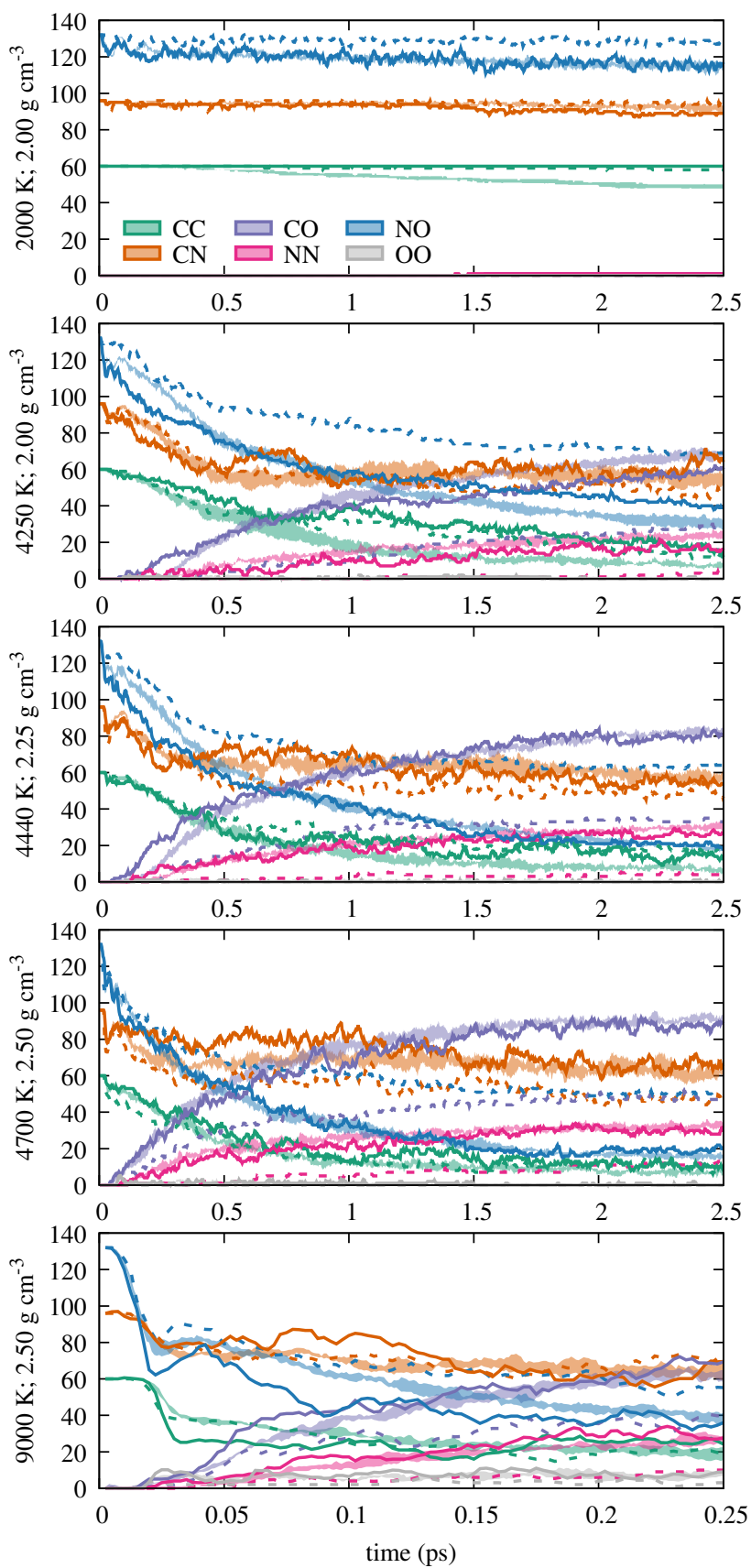

FIG. 4. Time evolution of bond counts in DNTF. DFT and Mio-1-1 predictions are given in solid and dashed lines, respectively, while shaded curves provide the spread about the Mio+ChIMES prediction in terms of $1^{\text {st }}$ and $3^{\text {rd }}$ quartiles. Bond types are indicated by the line colors, as given in the figure legend.

each of the three simulation methods. Slow reaction kinetics at $2000 \mathrm{~K}$ and the single independent simulation run for Mio-1-1 and DFT preclude conclusive remarks on suitability 

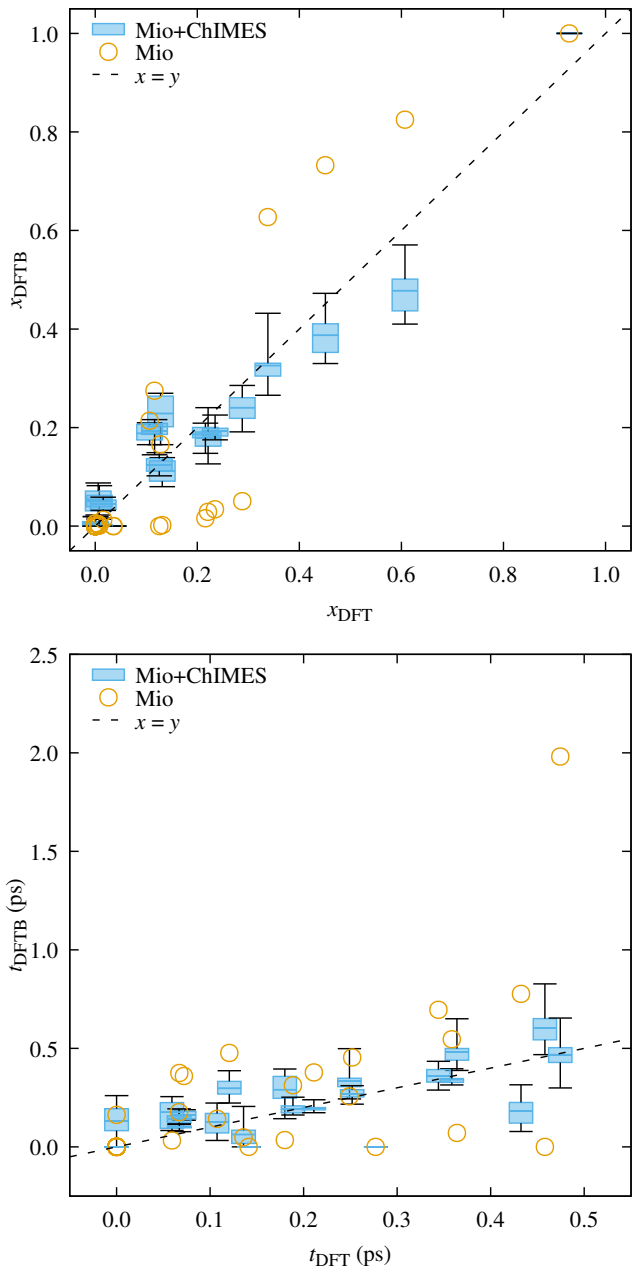

FIG. 5. Comparison of mole fractions (top) and lifetimes (bottom) for small species formed in DNTF over the range of training thermodynamic conditions, predicted by DFT, standard DFTB (Mio), and the DFTB-ChIMES model (Mio+ChIMES). Boxplots are used to convey the DFTBChIMES data, and provide the max/min values (indicated by the error bars), $1^{\text {st }}$ and $3^{\text {rd }}$ quartiles (indicated by the range of the shaded box), and the average value (indicated by the horizontal line within each box)

of Mio-1-1 and Mio+ChIMES for description of chemistry at this state point. However, we find that above $2000 \mathrm{~K}$, Mio-1-1 significantly over-predicts the number of $\mathrm{O}-\mathrm{N}$ bonds and under-predicts both $\mathrm{C}-\mathrm{O}$ and $\mathrm{N}-\mathrm{N}$ bond counts at any given time. In contrast, $\mathrm{Mio}+\mathrm{ChIMES}$ predictions are found to be in excellent agreement with DFT at all state points.

Chemistry was also evaluated in terms of predicted small molecule mole fractions and 

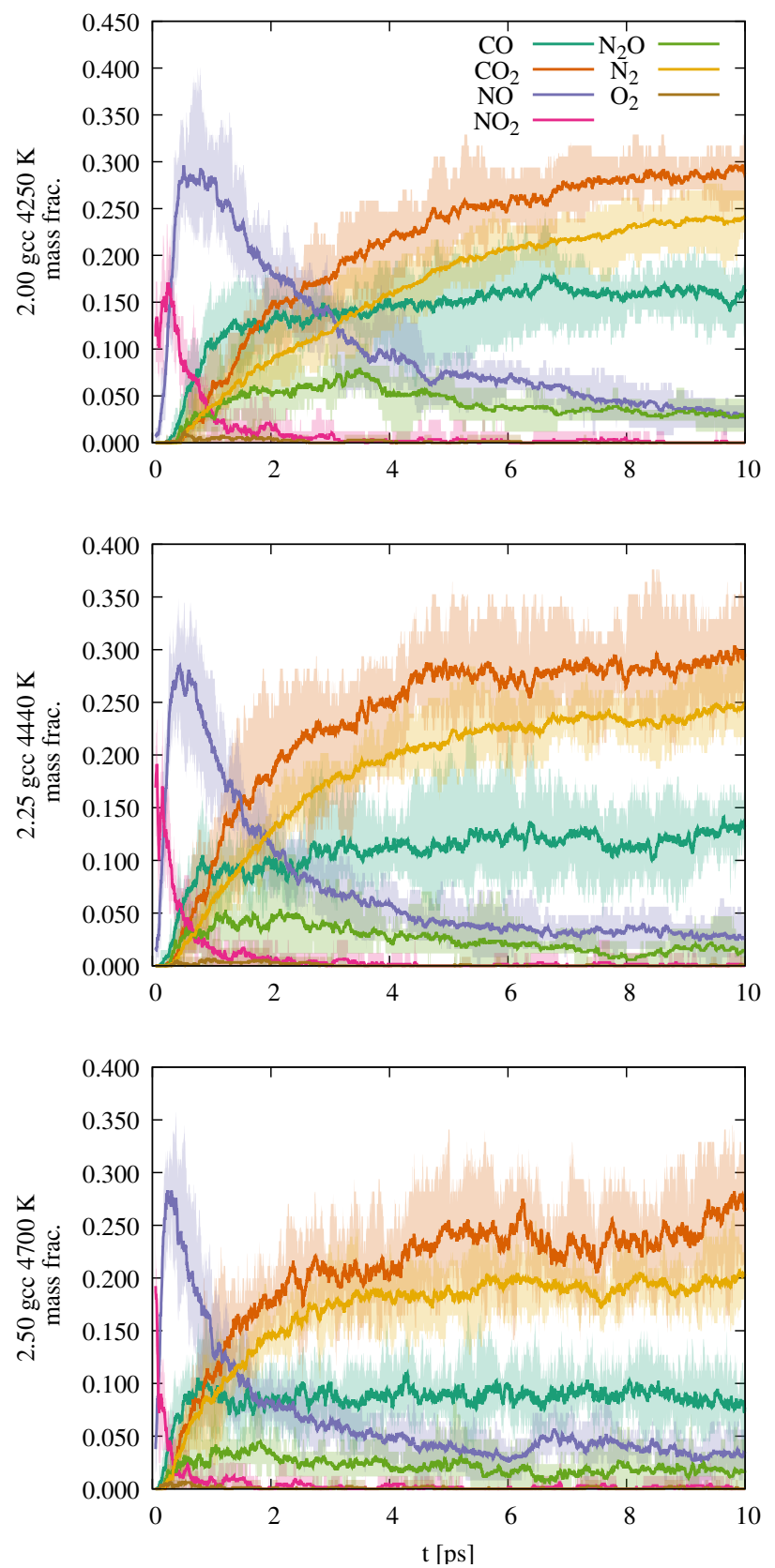

FIG. 6. Time resolved evolution of small molecule mass fractions during Mio+ChIMES NVT simulations. Solid lines provide average values across 8-independent simulations and the shaded region gives the corresponding $1^{\text {st }}$ and $3^{\text {rd }}$ quartiles.

lifetimes, where a species was said to exist if all atoms remained within the cutoffs provided above for 50 fs (i.e. long enough to observe one or two bond vibrations). The results, plotted in figure 5, compare mole fractions and lifetimes for $\mathrm{CO}, \mathrm{CO}_{2}, \mathrm{~N}_{2}, \mathrm{NO}, \mathrm{N}_{2} \mathrm{O}$, and $\mathrm{O}_{2}$, (i.e. small molecules characteristic to decomposition of DNTF) at each of the DFT state points 
indicated in Table I. Once again, we find that the Mio+ChIMES model yields enhanced recovery of DFT chemistry over Mio-1-1, though both are found to yield reasonable lifetime predictions. Overall, we find the present Mio+ChIMES model highly suited for studying DNTF under extreme conditions.

\section{B. Decomposition Kinetics}

We begin the study of DNTF subject to extreme conditions by investigating chemical decomposition kinetics at the three previously identified Hugoniot state points. For these simulations, systems were initialized in the molecular crystal state at the target density and temperature, and left to evolve over 10 ps. Experimentally, this would approximately correspond to single crystal DNTF suddenly heated under constant volume conditions, e.g. in a diamond anvil cell. We find that small molecule chemistry is similar across all three state points, with $\mathrm{N}_{x} \mathrm{O}_{y}$ species dominating at early times, and $\mathrm{CO}_{x}$ and $\mathrm{N}_{2}$ at late times. We note that other larger species with $\mathrm{C}_{x} \mathrm{~N}_{y} \mathrm{O}_{z}$ stoichiometry are also present in the Mio + ChIMES simulations (see Table II); hence, mass fractions in each plot do not sum to unity. From the relatively flat shape of each speciation trajectory, we conclude that the highest temperature state point in Figure 6 is closest to reaching what is likely to be a metastable state; the two lower-temperature systems appear to still be relaxing. Earlier work investigating the influence of oxygen balance on shockwave-driven chemical kinetics showed that in N-containing organic materials formation of equilibrium molecular products $\mathrm{N}_{2}$ and $\mathrm{CO}_{2}$ is significantly retarded in the presence of excess oxygen (i.e. in positive oxygen balance systems) due to formation of the "oxygen trapping" ion $\mathrm{NO}_{3}{ }^{49}$. Given that DNTF exhibits a negative oxygen balance, it is thus unsurprising that a lack of $\mathrm{NO}_{3}$ and generally uninhibited $\mathrm{N}_{2}$ and $\mathrm{CO}_{2}$ formation are observed; this is also in agreement with DFTB simulations for other materials with similar oxygen balance ${ }^{50}$. At the same time, we note that the speciation displays considerable complexity, with large $\mathrm{C}_{x} \mathrm{~N}_{y} \mathrm{O}_{z}$ products constituting a sizable mass fraction of the system at the end of the simulations, ranging from approximately 25 to $40 \%$ across the investigated densities. It is likely that these are the kinetic precursors to the carbon precipitates observed experimentally ${ }^{6}$, and therefore significant chemistry coupled with growth will occur on larger time scales. A similar effect was observed in high pressure and temperature simulations of carbon monoxide $(\mathrm{CO})^{51}$, where large $\mathrm{C}_{x} \mathrm{O}_{y}$ clusters were found to play an 
TABLE II. Average overall mass fraction of large $\mathrm{C}_{x} \mathrm{~N}_{y} \mathrm{O}_{z}$ species at the end of each set of simulations, and average mass fraction of each constituent atom type.

\begin{tabular}{|c|c|c|c|c|}
\hline Simulation & $x_{\text {other }}$ & $\% \mathrm{C}_{\text {other }}$ & $\% \mathrm{~N}_{\text {other }}$ & $\% \mathrm{O}_{\text {other }}$ \\
\hline $8.25 \mathrm{~km} / \mathrm{s}$ & 0.54 & 29 & 30 & 41 \\
\hline $8.50 \mathrm{~km} / \mathrm{s}$ & 0.63 & 28 & 31 & 41 \\
\hline $8.75 \mathrm{~km} / \mathrm{s}$ & 0.77 & 26 & 32 & 42 \\
\hline $9.00 \mathrm{~km} / \mathrm{s}$ & 0.76 & 26 & 34 & 40 \\
\hline $9.50 \mathrm{~km} / \mathrm{s}$ & 0.84 & 26 & 33 & 42 \\
\hline $2.00 \mathrm{~g} \mathrm{~cm}^{-3} / 4250 \mathrm{~K}$ & 0.25 & 33 & 38 & 29 \\
\hline $2.25 \mathrm{~g} \mathrm{~cm}^{-3} / 4440 \mathrm{~K}$ & 0.28 & 33 & 33 & 34 \\
\hline $2.50 \mathrm{~g} \mathrm{~cm}^{-3} / 4700 \mathrm{~K}$ & 0.40 & 31 & 34 & 35 \\
\hline
\end{tabular}

important role in early carbon condensation kinetics.

Thermochemical calculations ${ }^{46}$ at the simulated thermodynamic states (i.e. listed in Table I) indicate that the fully chemically equilibrated system corresponds to a mixture of small, stable molecules (e.g. $\mathrm{N}_{2}, \mathrm{CO}_{2}, \mathrm{CO}, \mathrm{NO}, \mathrm{NO}_{2}$, etc.) and carbon nanocondensates ${ }^{11,52}$. Indeed, experiments indicate that DNTF produces a significant amount of carbon particles with sizes from a few to 10 s of nanometers ${ }^{6}$. However, these are outside the length scales accessible to the current DFTB simulations, and therefore direct speciation comparisons between simulations and chemical equilibrium calculations are not necessarily edifying. A partially equilibrated calculation may be a better approximation of the simulated end states, but unfortunately the complex $\mathrm{C}_{x} \mathrm{~N}_{y} \mathrm{O}_{z}$ species identified in the simulations are difficult to capture in a thermochemical framework. Instead, we performed constrained thermochemical calculations in which a fraction of DNTF equal with the observed $\mathrm{C}_{x} \mathrm{~N}_{y} \mathrm{O}_{z}$ species mass fraction remains unreacted while the rest is a chemically equilibrated mixture of molecular products alone, without condensed carbon. These calculations are henceforth referred to as "partially-reacted" and should provide a chemistry picture somewhat closer to that observed at the present simulation scale. Consistent with the Mio+ChIMES calculations, these show that $\mathrm{CO}, \mathrm{CO}_{2}$, and $\mathrm{N}_{2}$ are the dominant species formed (see table III. In 
TABLE III. Small molecule mass fractions predicted via thermochemical calculation for the "partially reacted" system.

\begin{tabular}{c|ccc}
\hline \hline Simulation & $x_{\mathrm{CO}_{2}}$ & $x_{\mathrm{CO}}$ & $x_{\mathrm{N}_{2}}$ \\
\hline $2.00 \mathrm{~g} \mathrm{~cm}^{-3} / 4250 \mathrm{~K}$ & 0.24 & 0.31 & 0.31 \\
$2.25 \mathrm{~g} \mathrm{~cm}^{-3} / 4440 \mathrm{~K}$ & 0.22 & 0.29 & 0.29 \\
$2.50 \mathrm{~g} \mathrm{~cm}^{-3} / 4700 \mathrm{~K}$ & 0.18 & 0.23 & 0.23 \\
\hline \hline
\end{tabular}

particular, the calculated $\mathrm{N}_{2}$ mass fractions of $0.31,0.29$ and 0.23 at the 4250,4440 , and $4700 \mathrm{~K}$ state points, respectively, appear to match reasonably well the simulation values at the three thermodynamic points; $\mathrm{CO}_{2}$ fractions on the other hand are approximately $25 \%$ smaller and those for $\mathrm{CO}$ are roughly double the simulated results. Overall, the agreement is therefore only qualitative, likely due to the unfolding slow carbon kinetics signaled by the presence of large $\mathrm{C}_{x} \mathrm{~N}_{y} \mathrm{O}_{z}$ species, and the crude approximation used to capture their effect in the thermochemical estimates. Nevertheless, such comparisons bridging exceedingly different modeling methodologies can help translate insights and quantitative observations from atomistic simulations into equation of state and kinetic models applicable on much larger scales.

\section{Hugoniot Equation of State and Shock-Driven Chemistry}

In this section, we discuss prediction of the DNTF detonation state. For this work, we employed the extended Lagrangian Multiscale Shock Technique (MSST), which constrains the thermodynamic states of a MD simulation to those for a steady planar shockwave within continuum theory (i.e. simulating steady shock waves by constraining the stress and energy of a MD simulation to the Rayleigh line and the Hugoniot energy relations). This approach, which is discussed in greater detail elsewhere ${ }^{48,53,54}$, has been used to study shock properties of numerous materials, and here is used to estimate the DFT Chapman-Joguet (C-J) detonation state, Hugoniot curve, and shockwave-driven chemistry through our presently developed Mio+ChIMES proxy.

MSST simulations were run with a version of the LAMMPS software package locally 

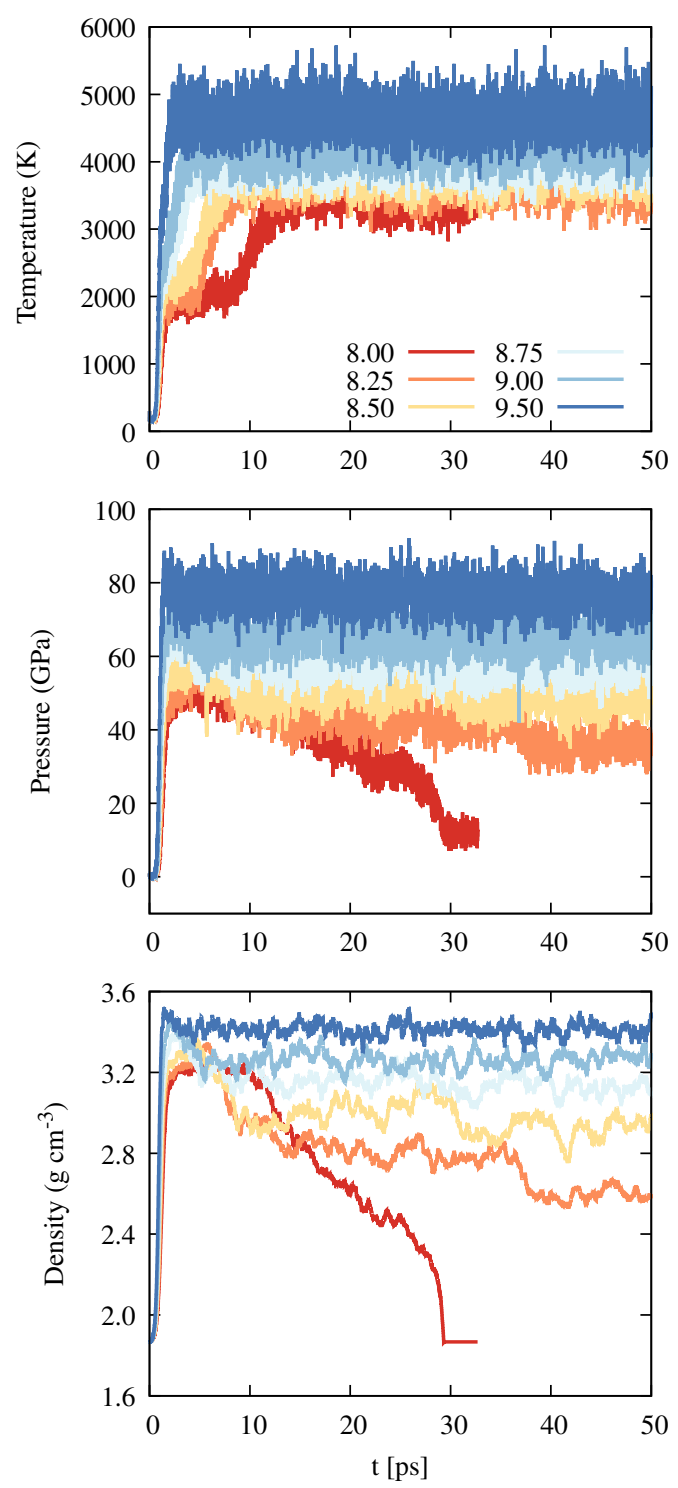

FIG. 7. Sample temperature, pressure, and density trajectories for MSST simulations.

modified to use DFTB + for evaluation of force, energy, and stress with the presently developed Mio+ChIMES model. These simulations comprised 176 atom systems initialized at a density of $1.86 \mathrm{~g} \mathrm{~cm}^{-3}$ and MSST cell-mass-like parameter and macroscopic explicit viscosity parameters of $Q=2.76 \times 10^{-13} \mathrm{~kg}^{2} \mathrm{~m}^{-4}$ and $m=0.02 \mathrm{~kg} \mathrm{~m}^{-1} \mathrm{~s}^{-1}$, respectively. Temperature, pressure, and density trajectories for simulations using shock velocities of 8.00 to $9.50 \mathrm{~km} \mathrm{~s}^{-1}$ are given in Figure 7 .

The C-J detonation velocity (i.e. the speed with which a steady state unsupported shock wave propagates through the material) establishes a lower limit on shock velocities for 


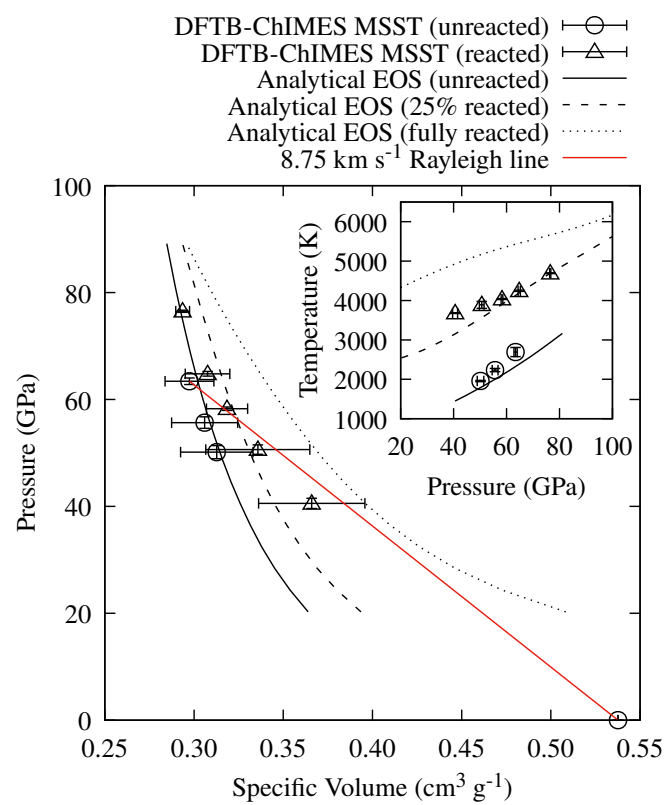

FIG. 8. DNTF shock Hugoniot states (reacted and unreacted) determined from the Mio+ChIMES MSST simulations compared with thermochemical estimates for a fully- and partially- chemically equilibrated system (see text), along with an analytic unreacted EOS based on high pressure experimental and simulation data.

which steady state solutions to the MSST equations exist. In the present work, we use this to estimate a lower-bound C-J velocity of at least $8.25 \mathrm{~km} \mathrm{~s}^{-1}$ for DNTF, since temperature, pressure, and density trajectories are found to diverge at $8.00 \mathrm{~km} \mathrm{~s}^{-1}$, and the latter is the lowest explored value for which stable simulations were obtained; the experimentally measured DNTF C-J detonation velocity ${ }^{55}$ at this density is $8.9 \mathrm{~km} \mathrm{~s}^{-1}$. We note that at shock velocities near but below the C-J points, MSST trajectory divergence can be slow, thus it is possible that the present trajectories at 8.50 and 8.75 could ultimately prove unstable if extended to substantially longer time scales (i.e. by orders of magnitude).

For shock velocities exhibiting steady solutions, the trajectories in Figure 7 indicate 3 distinct phases. The shockwave first drives the unreacted system to high density and pressure and intermediate temperature, corresponding to an unreacted shock Hugoniot point. Systems then begin to undergo decomposition chemistry, which drives expansion and an increase in temperature due to energy release. Finally, systems appear to relax to a quasi equilibrium state, corresponding to an incompletely reacted shock Hugoniot point. Figure 8 provides unreacted and reacted Hugoniot data resulting from these simulations in the classical $P$ vs 
$V$ representation. MSST simulations at a given shock velocity contribute a maximum of two data points (though unreacted points for shock velocities of 9.00 and $9.50 \mathrm{~km} \mathrm{~s}^{-1}$ could not be uniquely determined from the MSST trajectories due to rapid onset of reaction) and system evolution occurs along the Rayleigh line connecting the unreacted, reacted and initial states. Figure 9 provides the corresponding chemical evolution, and indicates a number of features common across all shock velocities. Similar to the NVT decomposition kinetics study, we find that $\mathrm{NO}$ and $\mathrm{NO}_{2}$ are the primary small molecules formed at early times, followed by $\mathrm{CO}_{2}$ and $\mathrm{N}_{2}$; interestingly, $\mathrm{NO}$ concentrations settle to progressively larger values for systems subjected to stronger shocks, possibly indicative of impeded chemistry at high densities. The kinetic time-scales of $\mathrm{CO}_{2}$ and $\mathrm{N}_{2}$ release are summarized in Table IV. These were obtained by fitting the $\mathrm{CO}_{2}$ and $\mathrm{N}_{2}$ trajectories to a rate equation of the form $x_{i, t}=x_{i, \infty}\left(1-\mathrm{e}^{-k_{i} \Delta t}\right)$, where $x_{i, t}$ and $x_{i, \infty}$ are the mass fraction of species $i$ at time $t$ and at nominal equilibrium, $k_{i}$ is the effective rate constant, $\Delta t=t-t_{0}$, and $t_{0}$ is the time point during the MSST trajectory at which chemistry begins. We note that at low shock velocities these rates are consistent with those reported from simulation studies of 1,3,5,7Tetranitro-1,3,5,7-tetrazoctan (i.e. HMX) under similar conditions ${ }^{50}$. Although the reacted states reached at the end of the shock simulations yield similar speciation to those from the NVT runs, they contain a significantly larger fraction of $\mathrm{C}_{x} \mathrm{~N}_{y} \mathrm{O}_{z}$ species, varying from approximately $55 \%$ at the lowest shock velocity to $85 \%$ at the largest one. Table II also indicates that carbon concentration in the $\mathrm{C}_{x} \mathrm{~N}_{y} \mathrm{O}_{z}$ material generated by shock compression is slightly enhanced relative to that of the DNTF molecule (e.g. 26-29\% compared to 23\%), while $\mathrm{N}$ concentration is reduced, and $\mathrm{O}$ concentration remains relatively unchanged (e.g. 30-33 compared to $36 \%$ and 40-42 compared to $41 \%$, respectively). In contrast, the $N V T$ simulations exhibit greater enhancements in C concentration in the material (e.g. 31-33\%), and $\mathrm{N}$ and $\mathrm{O}$ concentrations which are relatively unchanged and decreased, respectively. To highlight the impact of $\mathrm{C}_{x} \mathrm{~N}_{y} \mathrm{O}_{z}$ on the predicted shock Hugoniot, we also show in Fig. 8 both fully chemically equilibrated and $25 \%$ "partially reacted" thermochemical calculations (see previous description). The comparison between simulation results and thermochemical estimates illustrates the different stages of reactivity reached by the end of the simulations at different shock speeds, and suggests that the large $\mathrm{C}_{x} \mathrm{~N}_{y} \mathrm{O}_{z}$ species occurring after the break-up of the DNTF molecules likely hinder the advance of the system toward chemical equilibrium. High densities appear to play an especially important role in these initial pro- 
TABLE IV. Kinetics for $\mathrm{N}_{2}$ and $\mathrm{CO}_{2}$ formation (i.e. dominant small molecule species) from shock compressed DNTF predicted from Mio+ChIMES MSST simulations. Values for HMX ${ }^{50}$, which were obtained from previous simulation studies, are provided for comparison.

\begin{tabular}{c|cc|cc}
\hline \hline & \multicolumn{2}{|c|}{$\mathrm{N}_{2}$} & \multicolumn{2}{|c}{$\mathrm{CO}_{2}$} \\
\hline$u_{\mathrm{s}}\left(\mathrm{km} \mathrm{s}^{-1}\right)$ & $x_{\infty}$ & $k\left(\mathrm{ps}^{-1}\right)$ & $x_{\infty}$ & $k\left(\mathrm{ps}^{-1}\right)$ \\
\hline 8.25 & 0.206 & 0.09 & 0.264 & 0.09 \\
8.50 & 0.141 & 0.18 & 0.164 & 0.19 \\
8.75 & 0.102 & 0.24 & 0.114 & 0.25 \\
9.00 & 0.076 & 0.35 & 0.087 & 0.35 \\
9.50 & 0.076 & 0.39 & 0.065 & 0.57 \\
$\mathrm{HMX}$ & - & 0.08 & - & 0.05 \\
\hline \hline
\end{tabular}

cesses, which we believe precede the ultimate formation of carbon (likely liquid) clusters via densification, "impurity" ( $\mathrm{N}$ and $\mathrm{O}$ ) elimination and growth from these $\mathrm{C}_{x} \mathrm{~N}_{y} \mathrm{O}_{z}$ kinetic precursors. The relatively rapid release of $\mathrm{CO}_{2}$ compared to $\mathrm{N}_{2}$ appears consistent with the hypothesis ${ }^{6}$ that nitrogen is likelier than oxygen to become trapped in condensates formed from detonation of DNTF. In light of the previous discussion and the finite size effects further precluding the physical and chemical relaxation of the system into the fully reacted state containing a mixture of carbon particle and small molecular species, it is not surprising that Mio + ChIMES simulations yield reacted temperatures that are significantly lower than the thermochemical results - see Fig. 8(inset). (Differences in the unreacted temperatures are likely due to uncertainties in the thermal properties of DNTF.) It is clear that much larger length and time-scale simulations are necessary to capture the full system evolution and provide a clear picture of the dominant physicochemical mechanisms accompanying the shockwave compression of DNTF, and likely other carbon-rich energetic materials. Nevertheless, the current simulations yield the initial kinetic steps along the shock-constrained trajectory leading to the final chemical equilibrium state and can serve as checks for future studies employing efficient classical interatomic potentials.

Previous small-scale quantum-based studies have addressed the decomposition of other 

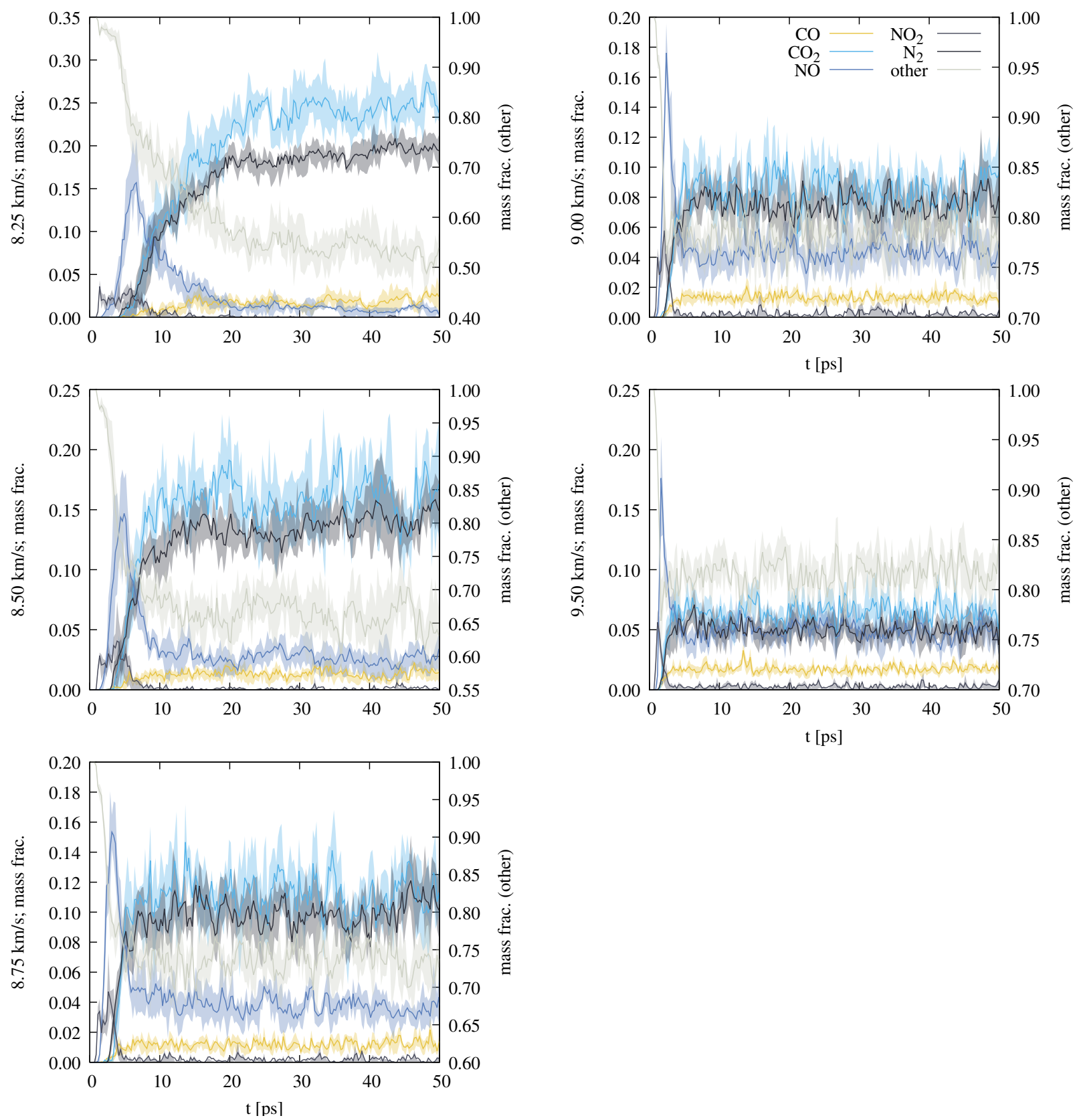

FIG. 9. Time resolved evolution of small molecule mass fractions during Mio+ChIMES MSST simulations. Solid lines provide average values across 8-independent simulations and the shaded region gives the corresponding $1^{\text {st }}$ and $3^{\text {rd }}$ quartiles. Mass fractions for small molecules and other material are given on the left and right $y$-axis, respectively.

energetic materials such as 1,3,5,7-tetranitro-1,3,5,7-tetrazoctane (HMX) ${ }^{50}$, which has a negative oxygen balance close to that of the DNTF studied here, and 2,4,6-triamino-1,3,5trinitrobenzene (TATB $)^{56}$, with a substantially larger magnitude oxygen balance. HMX de- 
composition at high density and temperature appears dominated by the production of small molecular species, while TATB was found to form very large, relatively stable C- and Ncontaining heterocyclic clusters. We learn here that sizable $\mathrm{C}_{x} \mathrm{~N}_{y} \mathrm{O}_{z}$ species are likely to be the kinetic precursors to the carbon particles that condense out of detonating DNTF ${ }^{6}$ We have also previously investigated reactivity in a $\mathrm{CO}$ fluid ${ }^{9,43,51}$, where carbon condensation at high pressures and temperatures proceeds through a reaction-driven phase separation mechanism $\left.{ }^{43,51,57}\right)$. Given that DNTF reaches detonation conditions similar to those yielding carbon condensates in $\mathrm{CO}$ (i.e. well into the liquid region of the carbon phase diagram), but it has a smaller (absolute) oxygen balance and also contains nitrogen, we believe that its study can shed further light on the complex interplay between carbon content, chemistry and thermodynamics in shocked energetic materials. Using relatively small system sizes we captured relevant early chemistry effects in DNTF; substantially larger scale simulations will be the subject of future work. We also note that expansion from the high pressure states studied here is likely to be a fruitful future direction, as the thermodynamic pathway along which the system is quenched can significantly impact carbon condensate size, chemistry and allotrope ${ }^{6}$.

\section{CONCLUSIONS}

In this work we demonstrate an effective ML approach in which ChIMES, a many-body reactive ML-IAP, is used to tune an approximate quantum mechanical model (i.e. the DFTB Mio-1-1 model) for description of reactivity in organic molecular materials under extreme conditions. We employ thermochemical calculations to guide generation of an initial training set through prediction of shock Hugoniot state points, and adopt an iterative approach wherein configurations from MSST simulations with the resulting Mio+ChIMES models are added to the training set to improve description of shock conditions and relaxation

to the reacted states. Resulting models, which were shown to maintain DFT-accuracy for description of system forces, energies, and stresses as well as structure, chemistry, and kinetics, were used to investigate decomposition and early shockwave driven chemistry in DNTF, a CNO energetic material known to form liquid carbon and nano-onion carbon condensates.

We find that, on the DFTB accessible time scales, an important aspect of DNTF chem- 
istry is the formation of $\mathrm{CO}_{2}, \mathrm{~N}_{2}$ and $\mathrm{CO}$. Following DNTF decomposition and significant production of these molecular products, the system appears to reach a metastable state earlier than other energetic materials with comparable oxygen balance such as HMX. This may be related to the absence of $\mathrm{H}_{2} \mathrm{O}$ as an available product, which mediates carbon and nitrogen chemistry at extreme conditions ${ }^{58}$. Though $\mathrm{NO}$ and $\mathrm{NO}_{2}$ formation delays $\mathrm{CO}_{2}, \mathrm{~N}_{2}$ and $\mathrm{CO}$ production to some extent, $\mathrm{NO}_{3}$ does not appear to play any role in inhibiting the chemistry, as it does in oxygen-rich and oxygen balanced materials. Crucially, we find that the occurrence and slow evolution of large $\mathrm{C}_{x} \mathrm{~N}_{y} \mathrm{O}_{z}$ species, which are likely precursors to the carbon condensates observed in experiments, is a major feature of shock-driven DNTF chemistry. Their detailed kinetics can only be characterized by simulations on time and length-scales larger than those accessible to DFTB. Nevertheless, the Mio+ChIMES MSST simulations yield a lower-bound for the $\mathrm{C}-\mathrm{J}$ detonation velocity and shock Hugoniot curves that are consistent with experiment and thermochemical estimates.

Ultimately, we find that the present Mio+ChIMES approach is well suited for the study of organic molecular materials under extreme conditions (i.e. where existing DFTB parameter sets are generally not applicable). Beyond providing a means of achieving long-timescale simulation with DFT-accuracy, this approach can greatly enhance efficiency in developing standalone ML-IAP (e.g. ChIMES) models, by serving as an effective (and rapid) means of generating DFT-quality data in cases where experimental results are scarce. Future work will focus on use of the present Mio+ChIMES DNTF model to generate reference data for development of a full ChIMES ML-IAP that can be leveraged in large scale (i.e. millions-of atoms) simulations and further extend simulation timescales to enable direct investigation of carbon condensation processes in carbon-rich organic materials.

\section{SUPPLEMENTARY MATERIAL}

The following files are available free of charge.

- SI.pdf: Additional discussion of the ChIMES model form and validation

- ChIMES_DNTF.2+3b.DFTB.params.txt: ChIMES parameters for the DFTB (Mio1-1) $E_{\text {corr }}$ developed in this work 


\section{ACKNOWLEDGMENTS}

This work was performed under the auspices of the U.S. Department of Energy by Lawrence Livermore National Laboratory under Contract DE-AC52-07NA27344 and was funded in part by the Joint Munitions Program under the Cheetah project and by the Advanced Simulation and Computing Program under the Physics and Engineering Models project. LLNL-JRNL-817132.

The data that supports the findings of this study are available within the article and its supplementary material..

\section{REFERENCES}

${ }^{1}$ Z. Martins, M. C. Price, N. Goldman, M. A. Sephton, and M. J. Burchell, "Shock synthesis of amino acids from impacting cometary and icy planet surface analogues," Nature Geoscience 6, 1045-1049 (2013).

${ }^{2}$ M. P. Kroonblawd, R. K. Lindsey, and N. Goldman, "Synthesis of functionalized nitrogencontaining polycyclic aromatic hydrocarbons and other prebiotic compounds in impacting glycine solutions," Chemical science 10, 6091-6098 (2019).

${ }^{3}$ N. Goldman, E. J. Reed, L. E. Fried, I.-F. W. Kuo, and A. Maiti, "Synthesis of glycinecontaining complexes in impacts of comets on early earth," Nature Chemistry 2, 949-954 (2010).

${ }^{4}$ N. R. Greiner, D. Phillips, J. Johnson, and F. Volk, "Diamonds in detonation soot," Nature 333, 440 (1988).

${ }^{5}$ V. N. Mochalin, O. Shenderova, D. Ho, and Y. Gogotsi, "The properties and applications of nanodiamonds," Nature nanotechnology 7, 11 (2012).

${ }^{6}$ M. Bagge-Hansen, S. Bastea, J. A. Hammons, M. H. Nielsen, L. Lauderbach, R. Hodgin, P. Pagoria, C. May, S. Aloni, A. Jones, et al., "Detonation synthesis of carbon nano-onions via liquid carbon condensation," Nature communications 10, 1-8 (2019).

${ }^{7}$ A. Krüger, F. Kataoka, M. a. a. Ozawa, T. Fujino, Y. Suzuki, A. Aleksenskii, A. Y. Vul, and E. Ōsawa, "Unusually tight aggregation in detonation nanodiamond: identification and disintegration," Carbon 43, 1722-1730 (2005). 
${ }^{8}$ V. Titov, V. Anisichkin, and I. Y. Mal'kov, "Synthesis of ultradispersed diamond in detonation waves," Combustion, Explosion and Shock Waves 25, 372-379 (1989).

${ }^{9}$ M. R. Armstrong, R. K. Lindsey, N. Goldman, M. H. Nielsen, E. Stavrou, L. E. Fried, J. M. Zaug, and S. Bastea, "Ultrafast shock synthesis of nanocarbon from a liquid precursor," Nature Communications 11, 1-7 (2020).

${ }^{10}$ W. Nellis, F. Ree, M. Van Thiel, and A. Mitchell, "Shock compression of liquid carbon monoxide and methane to 90 gpa (900 kbar)," The Journal of Chemical Physics 75, 30553063 (1981).

${ }^{11}$ S. Bastea, "Nanocarbon condensation in detonation," Scientific Reports 7, 42151 (2017).

${ }^{12}$ V. M. Titov, E. R. Pruuel, K. A. Ten, Luk'yanchikov, L. A. Merzhievskii, B. P. Tolochko, V. V. Zhulanov, and L. I. Shekhtman, "Experience of using synchrotron radiation for studying detonation processes," Combustion, Explosion, and Shock Waves 47, 615-626 (2011).

${ }^{13}$ M. Bagge-Hansen, L. Lauderbach, R. Hodgin, S. Bastea, L. Fried, A. Jones, T. van Buuren, D. Hansen, J. Benterou, C. May, T. Graber, B. J. Jensen, and T. M. Willey, "Measurement of carbon condensates using small-angle x-ray scattering during detonation of the high explosive hexanitrostilbene," Journal of Applied Physics 117, 245902 (2019).

${ }^{14}$ E. B. Watkins, K. A. Velizhanin, D. M. Dattelbaum, R. L. Gustavsen, T. D. Aslam, D. W. Podlesak, R. C. Huber, M. A. Firestone, B. S. Ringstrand, T. M. Willey, M. Bagge-Hansen, R. Hodgin, L. Lauderbach, T. van Buuren, N. Sinclair, P. A. Rigg, S. Seifert, and T. Gog, "Evolution of carbon clusters in the detonation products of the triaminotrinitrobenzene (tatb)-based explosive pbx 9502," Journal of Physical Chemistry C 121, 23129-23140 (2017).

${ }^{15}$ E. Schwegler, M. Sharma, F. Gygi, and G. Galli, "Melting of ice under pressure," Proceedings of the National Academy of Sciences 105, 14779-14783 (2008).

${ }^{16}$ K. K. Lee, L. R. Benedetti, R. Jeanloz, P. M. Celliers, J. H. Eggert, D. G. Hicks, S. J. Moon, A. Mackinnon, L. B. Da Silva, D. K. Bradley, et al., "Laser-driven shock experiments on precompressed water: Implications for "icy" giant planets," The Journal of Chemical Physics 125, 014701 (2006).

${ }^{17}$ N. Goldman, E. J. Reed, and L. E. Fried, "Quantum mechanical corrections to simulated shock hugoniot temperatures," The Journal of chemical physics 131, 204103 (2009).

${ }^{18}$ F. Dubnikova, R. Kosloff, J. Almog, Y. Zeiri, R. Boese, H. Itzhaky, A. Alt, and E. Keinan, 
"Decomposition of triacetone triperoxide is an entropic explosion," Journal of the American Chemical Society 127, 1146-1159 (2005).

${ }^{19}$ W. Kohn and L. J. Sham, "Self-consistent equations including exchange and correlation effects," Physical Review 140, A1133 (1965).

${ }^{20}$ N. Goldman, E. J. Reed, I.-F. W. Kuo, L. E. Fried, C. J. Mundy, and A. Curioni, "Ab initio simulation of the equation of state and kinetics of shocked water," The Journal of chemical physics 130, 124517 (2009).

${ }^{21}$ N. Goldman, L. E. Fried, and L. Koziol, "Using force-matched potentials to improve the accuracy of density functional tight binding for reactive conditions," Journal of Chemical Theory and Computation 11, 4530-4535 (2015).

${ }^{22}$ M. Elstner, D. Porezag, G. Jungnickel, J. Elsner, M. Haugk, T. Fraunheim, S. Suhai, and G. Seifert, "Self-consistent-charge density-functional tight-binding method for simulations of complex material properties," Physical Review B 58, 7260 (1998).

${ }^{23}$ G. Kresse and J. Hafner, "Ab initio molecular dynamics for liquid metals," Phys. Rev. B: Condens. Matter Mater. Phys. 47, 558 (1993).

${ }^{24} \mathrm{G}$. Kresse and J. Hafner, "Ab initio molecular-dynamics simulation of the liquid-metalamorphous-semiconductor transition in germanium," Phys. Rev. B: Condens. Matter Mater. Phys. 49, 14251 (1994).

${ }^{25}$ G. Kresse and J. Furthmüller, "Efficiency of ab-initio total energy calculations for metals and semiconductors using a plane-wave basis set," Comput. Mater. Sci. 6, 15-50 (1996).

${ }^{26} \mathrm{G}$. Kresse and J. Furthmüller, "Efficient iterative schemes for ab initio total-energy calculations using a plane-wave basis set," Phys. Rev. B: Condens. Matter Mater. Phys. 54, 11169 (1996).

${ }^{27}$ J. P. Perdew, K. Burke, and M. Ernzerhof, "Generalized gradient approximation made simple," Phys. Rev. Lett. 77, 3865 (1996).

${ }^{28}$ J. P. Perdew, K. Burke, and M. Ernzerhof, "Generalized gradient approximation made simple [erratum to phys. rev. lett. 77, 3865 (1996)]," Phys. Rev. Lett. 78, 1396-1396 (1997).

${ }^{29}$ P. E. Blöchl, "Projector augmented-wave method," Phys. Rev. B: Condens. Matter Mater. Phys. 50, 17953 (1994).

${ }^{30} \mathrm{G}$. Kresse and D. Joubert, "From ultrasoft pseudopotentials to the projector augmentedwave method," Phys. Rev. B: Condens. Matter Mater. Phys. 59, 1758 (1999). 
${ }^{31}$ S. Grimme, "Semiempirical gga-type density functional constructed with a long-range dispersion correction," J. Comput. Chem. 27, 1787-1799 (2006).

${ }^{32}$ S. Nosé, "A unified formulation of the constant temperature molecular dynamics methods," J. Chem. Phys. 81, 511-519 (1984).

${ }^{33}$ W. G. Hoover, "Canonical dynamics: equilibrium phase-space distributions," Phys. Rev. A: At., Mol., Opt. Phys. 31, 1695 (1985).

${ }^{34}$ M. Parrinello and A. Rahman, "Crystal structure and pair potentials: A moleculardynamics study," Physical review letters 45, 1196 (1980).

${ }^{35}$ M. Parrinello and A. Rahman, "Polymorphic transitions in single crystals: A new molecular dynamics method," Journal of Applied physics 52, 7182-7190 (1981).

${ }^{36}$ B. Hourahine, B. Aradi, V. Blum, F. Bonafe, A. Buccheri, C. Camacho, C. Cevallos, M. Deshaye, T. Dumitrică, A. Dominguez, et al., "Dftb+, a software package for efficient approximate density functional theory based atomistic simulations," The Journal of chemical physics 152, 124101 (2020).

${ }^{37}$ A. K. Rappé, C. J. Casewit, K. Colwell, W. A. Goddard III, and W. M. Skiff, "Uff, a full periodic table force field for molecular mechanics and molecular dynamics simulations," Journal of the American chemical society 114, 10024-10035 (1992).

${ }^{38}$ P. Koskinen and V. Mäkinen, "Density-functional tight-binding for beginners," Computational Materials Science 47, 237-253 (2009).

${ }^{39}$ M. Gaus, Q. Cui, and M. Elstner, "Dftb3: extension of the self-consistent-charge densityfunctional tight-binding method (scc-dftb)," Journal of chemical theory and computation 7, 931-948 (2011).

${ }^{40}$ R. K. Lindsey, L. E. Fried, and N. Goldman, "Chimes: A force matched potential with explicit three-body interactions for molten carbon," J. Chem. Theory Comput. 13, 62226229 (2017).

${ }^{41}$ N. Goldman, B. Aradi, R. K. Lindsey, and L. E. Fried, "Development of a multicenter density functional tight binding model for plutonium surface hydriding," Journal of chemical theory and computation 14, 2652-2660 (2018).

${ }^{42}$ R. K. Lindsey, L. E. Fried, and N. Goldman, "Application of the chimes force field to nonreactive molecular systems: Water at ambient conditions," J. Chem. Theory Comput. 15, 436-447 (2019).

${ }^{43}$ R. K. Lindsey, N. Goldman, L. E. Fried, and S. Bastea, "Many-body reactive force field de- 
velopment for carbon condensation in c/o systems under extreme conditions," The Journal of Chemical Physics 153, 054103 (2020).

${ }^{44}$ R. Lindsey, L. E. Fried, N. Goldman, and S. Bastea, "Active learning for robust, highcomplexity reactive atomistic simulations," (2020).

${ }^{45}$ R. Tibshirani, "Regression shrinkage and selection via the lasso," J. R. Statist. Soc. B 58, $267-288$ (1996).

${ }^{46}$ S. Bastea and L. Fried, Shockwave Science and Technology Reference Library, Vol. 6 (2012) pp. 1-31.

${ }^{47}$ V. P. Sinditskii, A. V. Burzhava, A. B. Sheremetev, and N. S. Aleksandrova, "Thermal and combustion properties of 3, 4-bis (3-nitrofurazan-4-yl) furoxan (dntf)," Propellants, Explosives, Pyrotechnics 37, 575-580 (2012).

${ }^{48}$ E. J. Reed, L. E. Fried, and J. Joannopoulos, "A method for tractable dynamical studies of single and double shock compression," Physical review letters 90, 235503 (2003).

${ }^{49} \mathrm{~N}$. Goldman and S. Bastea, "Nitrogen oxides as a chemistry trap in detonating oxygen-rich materials," J. Phys. Chem. A 118, 2897-2903 (2014).

${ }^{50}$ M. R. Manaa, L. E. Fried, C. F. Melius, M. Elstner, and T. Frauenheim, "Decomposition of hmx at extreme conditions: A molecular dynamics simulation," The Journal of Physical Chemistry A 106, 9024-9029 (2002).

${ }^{51}$ R. K. Lindsey, N. Goldman, L. E. Fried, , and S. Bastea, "Uncovering classicality in chemistry-coupled phase separation," In prep..

${ }^{52}$ J. Viecelli, S. Bastea, J. Glosli, and F. Ree, "Phase transformations of nanometer size carbon particles in shocked hydrocarbons and explosives," The Journal of Chemical Physics 115, 2730-2736 (2001).

${ }^{53}$ E. Reed, L. Fried, M. Manaa, and J. Joannopoulos, "Chemistry at extreme conditions," (2005).

${ }^{54}$ E. J. Reed, L. E. Fried, W. D. Henshaw, and C. M. Tarver, "Analysis of simulation technique for steady shock waves in materials with analytical equations of state," Physical Review E 74, 056706 (2006).

${ }^{55}$ Q.-h. Wang, "Properties of dntf-based melt-cast explosives (in chinese)," Chinese Journal of Explosives and Propellants 26, 57-59 (2003).

${ }^{56}$ M. R. Manaa, E. J. Reed, L. E. Fried, and N. Goldman, "Nitrogen-rich heterocycles as reactivity retardants in shocked insensitive explosives," Journal of the American Chemical 
Society 131, 5483-5487 (2009).

${ }^{57}$ T. C. Leonhardi and B. Militzer, "Ab initio simulations of liquid carbon monoxide at high pressure," High Energy Density Physics 22, 41-45 (2017).

${ }^{58}$ C. Wu, L. Fried, L. Yang, N. Goldman, and S. Bastea, "Catalytic behavior of dense hot water," Nature chemistry 1, 57-62 (2009). 


\title{
Supplementary Information: Investigating
} 3,4-bis(3-nitrofurazan-4-yl)furoxan Detonation with a Rapidly Tuned Density Functional Tight Binding Model

\author{
Rebecca K. Lindsey, ${ }^{* \dagger}$ Sorin Bastea, ${ }^{\dagger}$ Nir Goldman, ${ }^{\dagger, \dagger}$ and Laurence E. Fried ${ }^{\dagger}$ \\ $\dagger$ †hysical and Life Sciences Directorate, Lawrence Livermore National Laboratory, \\ Livermore, California 94550, United States \\ $\ddagger$ Department of Chemical Engineering, University of California, Davis, California 95616, \\ United States \\ E-mail: lindsey11@llnl.gov \\ Phone: +1-925-422-0915
}




\section{Additional ChIMES Details}

The Chebyshev polynomials comprising ChIMES interactions take a transformed pair distance, i.e. $s_{i j}^{e_{i} e_{j}}$, as their argument. In contrast to a "standard" pair distance (e.g. $r_{i j}$, which gives the distance between two atoms $i$ and $j), s_{i j}^{e_{i} e_{j}}$ gives a pair distance transformed to span the [-1,1] domain over which Chebyshev polynomials are defined, and is obtained through: ${ }^{1}$

$$
\begin{gathered}
x_{i j}^{e_{i} e_{j}}=\exp \left(-r_{i j}^{e_{i} e_{j}} / \lambda^{e_{i} e_{j}}\right) \\
x_{\mathrm{avg}}^{e_{i} e_{j}}=0.5\left(x_{\mathrm{c}, \text { out }}^{e_{i} e_{j}}+x_{\mathrm{c}, \text { in }}^{e_{i} e_{j}}\right) \\
x_{\mathrm{diff}}^{e_{i} e_{j}}=0.5\left|x_{\mathrm{c}, \text { out }}^{e_{i} e_{j}}-x_{\mathrm{c}, \text { in }}^{e_{i} e_{j}}\right| \\
s_{i j}^{e_{i} e_{j}}=\left.\left(x_{i j}^{e_{i} e_{j}}-x_{\mathrm{avg}}^{e_{i} e_{j}}\right)\right|_{\mathrm{diff}} ^{e_{i} e_{j}}
\end{gathered}
$$

where $e_{i}$ is the element type of atom $i, s_{i j}^{e_{i} e_{j}}$ is the pair distance and $\lambda^{e_{i} e_{j}}$ can be considered a characteristic bonding distance, typically set to the location of the first peak in the DFT radial distribution function for the $e_{i} e_{j}$ atom pair type, and $r_{\mathrm{c}, \text { in }}^{e_{i} e_{j}} / r_{\mathrm{c}, \text { out }}^{e_{i} e_{j}}$ are the corresponding inner/outer cutoff radii. We note that Eq. 1 enforces a natural decrease in interaction strength as distance is increased, and increasing $\lambda^{e_{i} e_{j}}$ has the effect of decreasing the rate of interaction decay as $r_{\mathrm{c}, \text { out }}^{e_{i} e_{j}}$ is approached, in a Morse-like ${ }^{1}$ fashion.

As described in the main text, ChIMES interactions also include a smoothing function (i.e. $f_{\mathrm{s}}^{e_{i} e_{j}}$ ) which ensures each term in the polynomial series decays to zero at the outer cutoff, without any discontinuities. The smoothing function takes on the form:

$$
f_{\mathrm{s}}^{e_{i} e_{j}}\left(r_{i j}\right)= \begin{cases}0, & \text { if } r_{i j}>r_{\mathrm{c}, \text { out }}^{e_{i} e_{j}} \\ 1, & \text { if } r_{i j}<d_{\mathrm{t}} \\ \frac{1}{2}+\frac{1}{2} \sin \left(\pi\left[\frac{r_{i j}-d_{\mathrm{t}}}{r_{\mathrm{c}, e_{j}}}\right]+\frac{\pi}{2}\right), & \text { otherwise }\end{cases}
$$

where the threshold distance is given by $d_{\mathrm{t}}=r_{\mathrm{c} \text {,out }}^{m_{1}(y)}\left(1-f_{\mathrm{O}}\right)$, and $f_{\mathrm{O}}$ is a value in $[0,1]$ taken to be 0.5 , here. This function exhibits a smooth step, allowing the interaction energy to remain unmodified by the smoothing function for all $r_{i j}<d_{\mathrm{t}}$. We note that this smoothing function is particularly useful for manybody interactions of large $n$, where the product of $\left(\begin{array}{c}n \\ 2\end{array}\right) f_{\mathrm{s}}^{e_{i} e_{j}}\left(r_{i j}\right)$ factors is used, and can otherwise severely reduce energy contributions to the total energy for $n>2$.

Finally, ChIMES two-body interactions include a penalty function which discourages the system from sampling pair distances below the inner cutoffs. The penalty function is given by:

$$
f_{\mathrm{p}}^{e_{i} e_{j}}\left(r_{i j}\right)= \begin{cases}A_{\mathrm{p}}^{e_{i} e_{j}}\left(r_{\mathrm{c}, \text { in }}^{e_{i} e_{j}}+d_{\mathrm{p}}^{e_{i} e_{j}}-r_{i j}\right)^{3}, & \text { if } r_{i j}<r_{\mathrm{c}, \text { in }}^{e_{i} e_{j}}+d_{\mathrm{p}} \\ 0, & \text { otherwise }\end{cases}
$$

where $A_{\mathrm{p}}^{e_{i} e_{j}}, d_{\mathrm{p}}^{e_{i} e_{j}}$, and $r_{\mathrm{c}, \text { in }}^{e_{i} e_{j}}$ are the penalty function strength, kick-in distance, and the inner cutoff for atom types $e_{i}$ and $e_{j}$, respectively.

\section{Additional Validation Data}

The ChIMES-corrected DFTB model (i.e. Mio+ChIMES) discussed in the main text evaluated in terms of the radial pair distribution functions (RDFs)for each atom pair type at each training thermodynamic state point. Figure 1 provides RDFs for DNTF predicted by DFT, "standard" DFTB (i.e. Mio-1-1), and the presently developed Mio+ChIMES model. In general, we find both DFTB models in good agreement with DFT, though the Mio + ChIMES model tends to yield better performance under more extreme conditions (e.g. $T \geq 4440$ ). 


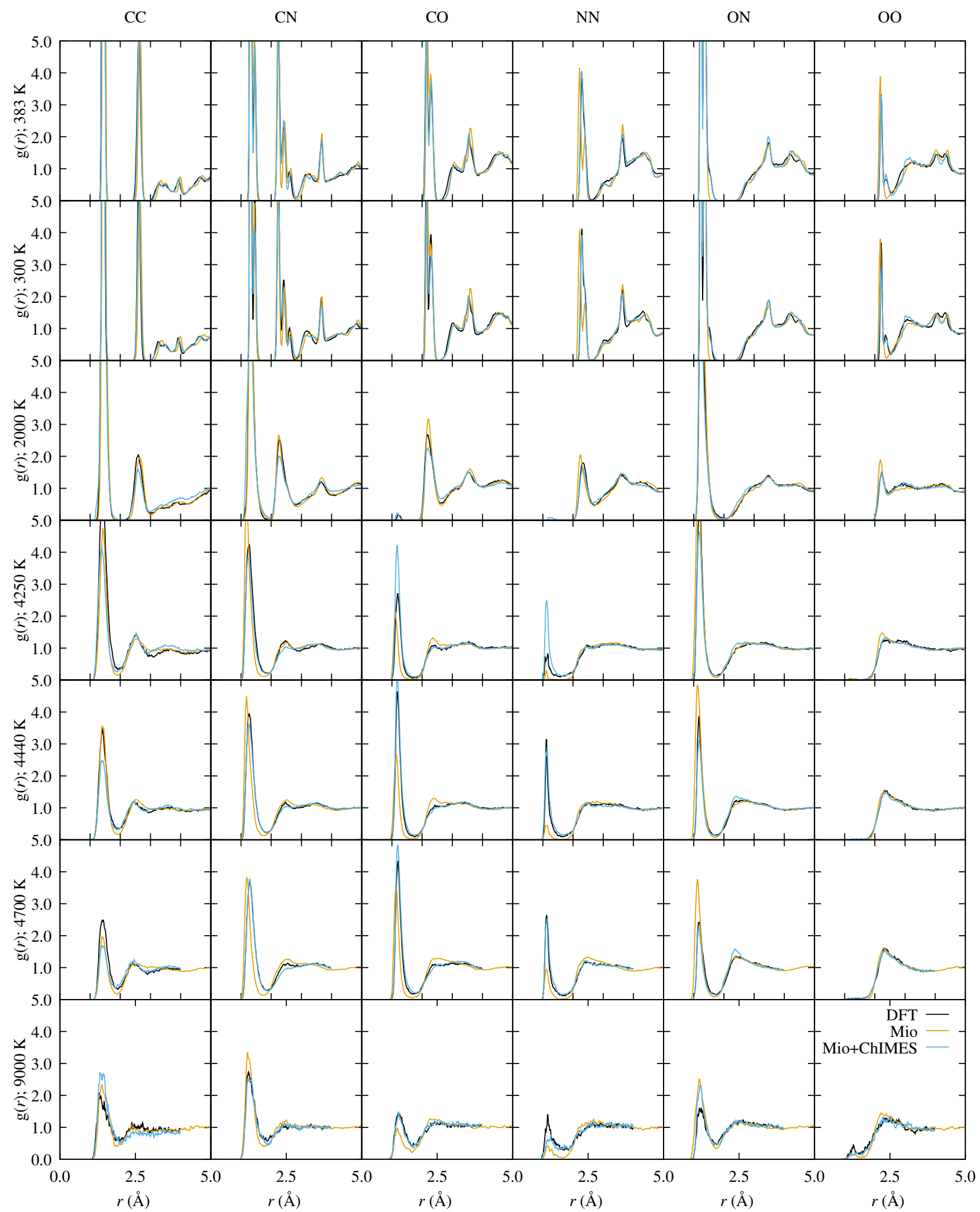

Figure 1: Radial pair distribution functions predicted by DFT, Mio-1-1 ("Mio"), and the presently developed Mio+ChIMES models. 


\section{References}

(1) Braams, B. J.; Bowman, J. M. Permutationally invariant potential energy surfaces in high dimensionality. Int. Rev. Phys. Chem. 2009, 28, 577-606. 
! This model is only intended for DNTF from approximately 300 to $9000 \mathrm{~K}$ and 1.86 to $3.4 \mathrm{gcc}$ ! This model is not applicable to other atom type ratios

USECOUL: false

FITCOUL: false

USEPOVR: false

FITPOVR: false

USE3BCH: true

USE4BCH: false

PAIRTYP: CHEBYSHEV $1280-11$

ATOM TYPES: 3

$\begin{array}{llll}\text { \# TYPEIDX \# } & \text { \# ATM_TYP \# } & \text { \# ATMCHRG \# } & \text { \# ATMMASS \# } \\ 0 & \text { C } & 0 & 12.0107 \\ 1 & \text { N } & 0 & 14.0067 \\ 2 & \text { O } & 0 & 15.999\end{array}$

ATOM PAIRS: 6

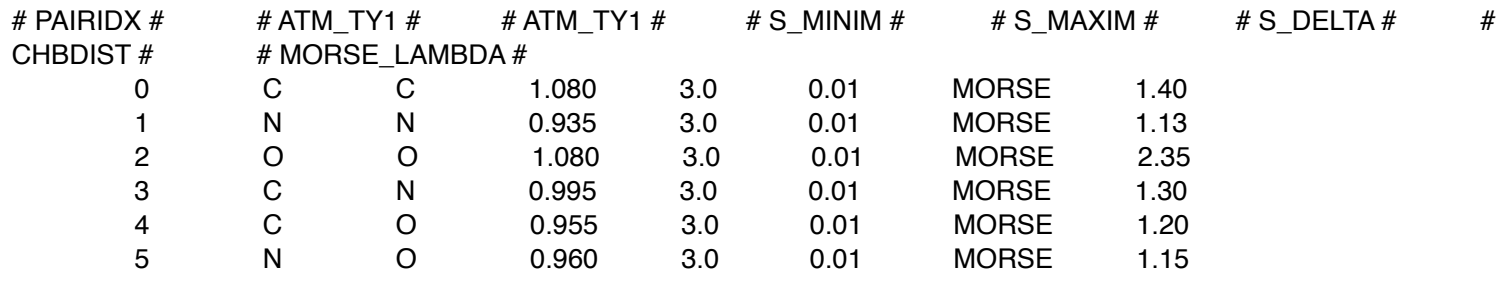

FCUT TYPE: TERSOFF 0.5

PAIR CHEBYSHEV PENALTY DIST: 0.02

PAIR CHEBYSHEV PENALTY SCALING: 1000000.0

SPECIAL 3B S_MAXIM: SPECIFIC 10

CCCCCC CC CC CC 3.000003 .000003 .00000

CCCNCN CC CN CN 3.000003 .000003 .00000

CCCOCO CC CO CO 3.000003 .000003 .00000

CNCNNN CN CN NN 3.000003 .000003 .00000

CNCONO CN CO NO 3.000003 .000003 .00000

COCOOO CO CO OO 3.000003 .000003 .00000

NNNNNN NN NN NN 3.000003 .000003 .00000

NNNONO NN NO NO 3.000003 .000003 .00000

NONOOO NO NO OO 3.000003 .000003 .00000

OOOOOO OO OO OO 3.000003 .000003 .00000

ATOM PAIR TRIPLETS: 10

ATOM PAIR QUADRUPLETS: 0

PAIR CHEBYSHEV PARAMS

PAIRTYPE PARAMS: $0 \mathrm{C} \mathrm{C}$
$0-22.3416405279$
19.991423956054
2 -6.975426943187
3 1.522138916278
$4-0.8607258377407$
52.107066974107
$6-2.450494382222$
70.6075393845831
$8-0.4032939229531$
90.5770468647435
$10-0.6855333350413$ 
110.5254109036754

PAIRTYPE PARAMS: $1 \mathrm{~N} \mathrm{~N}$

$\begin{array}{ll}0 & 6.032592456936 \\ 1 & 11.57081911618 \\ 2 & 2.614242090383 \\ 3 & -7.917005724114 \\ 4 & 8.229418287383 \\ 5 & 0.2224753695561 \\ 6 & 0.9239671094822 \\ 7 & -1.174713847289 \\ 8 & 3.24614691441 \\ 9 & -2.296458575943 \\ 10 & 0.887089625148 \\ 11 & -0.0009331334258886\end{array}$

PAIRTYPE PARAMS: 200

$0-31.68506413688$

118.21328634329

$2-5.928017516687$

313.15602372867

$4-11.6648020585$

57.949578712528

$6-3.487756504078$

73.502174389637

8 -3.171502434946

91.322516316714

$10-0.6397425233484$

110.3873698922037

PAIRTYPE PARAMS: $3 \mathrm{C} \mathrm{N}$

$0-3.032288794861$

111.85464638299

23.689531382371

3-3.000940965044

41.175906583971

51.275267355812

$6-0.2233697483747$

$7-0.4256839494941$

80.5948275266625

90.08631529090629

$10-0.1971497557096$

110.2147071116057

PAIRTYPE PARAMS: $4 \mathrm{C} \mathrm{O}$
$0-9.091927806649$
120.35329281444
23.969231583633
3 3.169556820228
$4-1.213055631955$
52.831566572585
$6-1.065828659787$
71.499037708973
80.5663897970059
90.2767692441393
$10-0.1790891237699$
110.4422505706705

PAIRTYPE PARAMS: $5 \mathrm{~N} \mathrm{O}$

013.0721713383

16.240612160136 


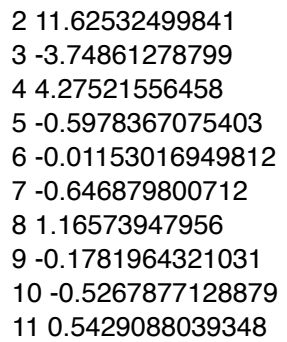

\section{TRIPLET CHEBYSHEV PARAMS}

TRIPLETTYPE PARAMS:

INDEX: O ATOMS: C C C

PAIRS: CC CC CC UNIQUE: -1 TOTAL: 423

index I powers I equiv index I param index I parameter

00110013.93814477678

11010013.93814477678

21100013.93814477678

$301231-3.022816795842$

$410231-3.022816795842$

$502131-3.022816795842$

$620131-3.022816795842$

$712031-3.022816795842$

$821031-3.022816795842$

$901392-1.445184973737$

$1010392-1.445184973737$

$1103192-1.445184973737$

$1230192-1.445184973737$

$1313092-1.445184973737$

$1431092-1.445184973737$

$15014153-12.93198698576$

$16104153-12.93198698576$

$17041153-12.93198698576$

$18401153-12.93198698576$

$19140153-12.93198698576$

$20410153-12.93198698576$

$21016275-2.681841907453$

$22106275-2.681841907453$

$23061275-2.681841907453$

$24601275-2.681841907453$

$25160275-2.681841907453$

$26610275-2.681841907453$

270173361.875359207502

281073361.875359207502

290713361.875359207502

307013361.875359207502

311703361.875359207502

327103361.875359207502

$33022397-3.406756593933$

$34202397-3.406756593933$

$35220397-3.406756593933$

$36023428-1.420654567067$

$37203428-1.420654567067$

$38032428-1.420654567067$ $39302428-1.420654567067$ $40230428-1.420654567067$ $41320428-1.420654567067$ $42024489-3.687050398721$ $43204489-3.687050398721$ $44042489-3.687050398721$ $45402489-3.687050398721$ $46240489-3.687050398721$ $47420489-3.687050398721$ 
4802554100.08826600876929 4920554100.08826600876929 5005254100.08826600876929 5150254100.08826600876929 5225054100.08826600876929 5352054100.08826600876929 $540266011-2.492644875064$ $552066011-2.492644875064$ $560626011-2.492644875064$ $576026011-2.492644875064$ $582606011-2.492644875064$ $596206011-2.492644875064$ $600276612-1.970242471024$ $612076612-1.970242471024$ $620726612-1.970242471024$ $637026612-1.970242471024$ $642706612-1.970242471024$ $657206612-1.970242471024$ $660337213-4.534261069854$ $673037213-4.534261069854$ $683307213-4.534261069854$ $690347514-5.408878651501$ $703047514-5.408878651501$ $710437514-5.408878651501$ $724037514-5.408878651501$ $733407514-5.408878651501$ $744307514-5.408878651501$ 7503581150.8010788665199 7630581150.8010788665199 7705381150.8010788665199 7850381150.8010788665199 7935081150.8010788665199 8053081150.8010788665199 $810368716-0.2235369968836$ $823068716-0.2235369968836$ $830638716-0.2235369968836$ $846038716-0.2235369968836$ $853608716-0.2235369968836$ $866308716-0.2235369968836$ 8703793172.954415890781 8830793172.954415890781 8907393172.954415890781 9070393172.954415890781 9137093172.954415890781 9273093172.954415890781 93045102192.663568459485 94405102192.663568459485 95054102192.663568459485 96504102192.663568459485 97450102192.663568459485 98540102192.663568459485 99046108201.681209602811 100406108201.681209602811 101064108201.681209602811 102604108201.681209602811 103460108201.681209602811 104640108201.681209602811 $10504711421-3.662065745875$ $10640711421-3.662065745875$ $10707411421-3.662065745875$ $10870411421-3.662065745875$ $10947011421-3.662065745875$ $11074011421-3.662065745875$ 111055120223.869095915388 112505120223.869095915388 113550120223.869095915388 
114056123231.364957086817 115506123231.364957086817 116065123231.364957086817 117605123231.364957086817 118560123231.364957086817 119650123231.364957086817 120057129242.757863228468 121507129242.757863228468 122075129242.757863228468 123705129242.757863228468 124570129242.757863228468 125750129242.757863228468 126066135252.129469588882 127606135252.129469588882 128660135252.129469588882 $12906713826-1.406629694597$ $13060713826-1.406629694597$ $13107613826-1.406629694597$ $13270613826-1.406629694597$ $13367013826-1.406629694597$ $13476013826-1.406629694597$ 135077144272.217587685735 136707144272.217587685735 137770144272.217587685735 $13811315130-18.9435581649$ $13913115130-18.9435581649$ $14031115130-18.9435581649$ 141114154315.90024522508 142141154315.90024522508 143411154315.90024522508 1441171633411.64319763918 1451711633411.64319763918 1467111633411.64319763918 $14712216635-2.363338569032$ $14821216635-2.363338569032$ $14922116635-2.363338569032$ $15012316936-3.067768872605$ $15121316936-3.067768872605$ $15213216936-3.067768872605$ $15331216936-3.067768872605$ $15423116936-3.067768872605$ $15532116936-3.067768872605$ $15612417537-0.7819714583635$ $15721417537-0.7819714583635$ $15814217537-0.7819714583635$ $15941217537-0.7819714583635$ $16024117537-0.7819714583635$ $16142117537-0.7819714583635$ $16212719340-2.144334334145$ $16321719340-2.144334334145$ $16417219340-2.144334334145$ $16571219340-2.144334334145$ $16627119340-2.144334334145$ $16772119340-2.144334334145$ 1681342024214.11227535115 1693142024214.11227535115 1701432024214.11227535115 1714132024214.11227535115 1723412024214.11227535115 1734312024214.11227535115 174135208431.349624071909 175315208431.349624071909 176153208431.349624071909 177513208431.349624071909 178351208431.349624071909 179531208431.349624071909 
$18013621444-0.7238330655984$ $18131621444-0.7238330655984$ $18216321444-0.7238330655984$ $18361321444-0.7238330655984$ $18436121444-0.7238330655984$ $18563121444-0.7238330655984$ 186137220452.136018506613 187317220452.136018506613 188173220452.136018506613 189713220452.136018506613 190371220452.136018506613 191731220452.136018506613 192144226464.924855030296 193414226464.924855030296 194441226464.924855030296 195146235481.79133027103 196416235481.79133027103 197164235481.79133027103 198614235481.79133027103 199461235481.79133027103 200641235481.79133027103 $20114724149-0.01791078801475$ $20241724149-0.01791078801475$ $20317424149-0.01791078801475$ $20471424149-0.01791078801475$ $20547124149-0.01791078801475$ $20674124149-0.01791078801475$ 207156250515.454649835 208516250515.454649835 209165250515.454649835 210615250515.454649835 211561250515.454649835 212651250515.454649835 213157256524.294279431392 214517256524.294279431392 215175256524.294279431392 216715256524.294279431392 217571256524.294279431392 218751256524.294279431392 219166262538.903114270332 220616262538.903114270332 221661262538.903114270332 222167265543.175835993802 223617265543.175835993802 224176265543.175835993802 225716265543.175835993802 226671265543.175835993802 227761265543.175835993802 228177271556.505114614994 229717271556.505114614994 230771271556.505114614994 $23122227456-7.666128858921$ 232223275572.764292593954 233232275572.764292593954 234322275572.764292593954 $23522427858-10.40803721758$ $23624227858-10.40803721758$ $23742227858-10.40803721758$ $23822628460-5.574756669583$ $23926228460-5.574756669583$ $24062228460-5.574756669583$ $24122728761-7.369475686209$ $24227228761-7.369475686209$ $24372228761-7.369475686209$ 244234293632.476282111463 245324293632.476282111463 
246243293632.476282111463 247423293632.476282111463 248342293632.476282111463 249432293632.476282111463 $25023529964-0.6700551216241$ $25132529964-0.6700551216241$ $25225329964-0.6700551216241$ $25352329964-0.6700551216241$ $25435229964-0.6700551216241$ $25553229964-0.6700551216241$ 256236305650.7808923134823 257326305650.7808923134823 258263305650.7808923134823 259623305650.7808923134823 260362305650.7808923134823 261632305650.7808923134823 $26224431767-4.357902785923$ $26342431767-4.357902785923$ $26444231767-4.357902785923$ 265245320684.444112967149 266425320684.444112967149 267254320684.444112967149 268524320684.444112967149 269452320684.444112967149 270542320684.444112967149 271246326690.4557055010626 272426326690.4557055010626 273264326690.4557055010626 274624326690.4557055010626 275462326690.4557055010626 276642326690.4557055010626 $27724733270-8.856093830374$ $27842733270-8.856093830374$ $27927433270-8.856093830374$ $28072433270-8.856093830374$ $28147233270-8.856093830374$ $28274233270-8.856093830374$ 283256341720.9836603222206 284526341720.9836603222206 285265341720.9836603222206 286625341720.9836603222206 287562341720.9836603222206 288652341720.9836603222206 289257347730.9402110047442 290527347730.9402110047442 291275347730.9402110047442 292725347730.9402110047442 293572347730.9402110047442 294752347730.9402110047442 295266353742.332904247898 296626353742.332904247898 297662353742.332904247898 $29826735675-3.368019706742$ $29962735675-3.368019706742$ $30027635675-3.368019706742$ $30172635675-3.368019706742$ $30267235675-3.368019706742$ $30376235675-3.368019706742$ 304277362762.681973791296 305727362762.681973791296 306772362762.681973791296 3073333657717.65926738312 308334366789.777216586082 309343366789.777216586082 310433366789.777216586082 $31133536979-2.355475559444$ 
$31235336979-2.355475559444$ $31353336979-2.355475559444$ $31433637280-6.010706319764$ $31536337280-6.010706319764$ $31663337280-6.010706319764$ $31733737581-3.818250525935$ $31837337581-3.818250525935$ $31973337581-3.818250525935$ $32034538183-5.811968732141$ $32143538183-5.811968732141$ $32235438183-5.811968732141$ $32353438183-5.811968732141$ $32445338183-5.811968732141$ $32554338183-5.811968732141$ $32634638784-0.5701586691002$ $32743638784-0.5701586691002$ $32836438784-0.5701586691002$ $32963438784-0.5701586691002$ $33046338784-0.5701586691002$ $33164338784-0.5701586691002$ $33234739385-1.100436833034$ $33343739385-1.100436833034$ $33437439385-1.100436833034$ $33573439385-1.100436833034$ $33647339385-1.100436833034$ $33774339385-1.100436833034$ $33835539986-10.00695428574$ $33953539986-10.00695428574$ $34055339986-10.00695428574$ $34135640287-0.01506722937171$ $34253640287-0.01506722937171$ $34336540287-0.01506722937171$ $34463540287-0.01506722937171$ $34556340287-0.01506722937171$ $34665340287-0.01506722937171$ $34735740888-1.373412380224$ $34853740888-1.373412380224$ $34937540888-1.373412380224$ $35073540888-1.373412380224$ $35157340888-1.373412380224$ $35275340888-1.373412380224$ 353366414896.282882792132 354636414896.282882792132 355663414896.282882792132 356367417901.870060450334 357637417901.870060450334 358376417901.870060450334 359736417901.870060450334 360673417901.870060450334 361763417901.870060450334 362377423913.320500758763 363737423913.320500758763 364773423913.320500758763 $36544442692-3.082414196374$ 366445427932.584653264593 367454427932.584653264593 368544427932.584653264593 $36944643094-0.4333520918582$ $37046443094-0.4333520918582$ $37164443094-0.4333520918582$ $37244743395-8.237136449926$ $37347443395-8.237136449926$ $37474443395-8.237136449926$ $37545543696-9.493713950906$ $37654543696-9.493713950906$ $37755443696-9.493713950906$ 
378456439972.264584651644 379546439972.264584651644 380465439972.264584651644 381645439972.264584651644 382564439972.264584651644 383654439972.264584651644 $38445744598-0.9895480085202$ $38554744598-0.9895480085202$ $38647544598-0.9895480085202$ $38774544598-0.9895480085202$ $38857444598-0.9895480085202$ $38975444598-0.9895480085202$ $390467454100-1.808492715692$ $391647454100-1.808492715692$ $392476454100-1.808492715692$ $393746454100-1.808492715692$ $394674454100-1.808492715692$ $395764454100-1.808492715692$ 3964774601011.714694385873 3977474601011.714694385873 3987744601011.714694385873 $399555463102-9.059238230698$ 4005564641032.174250906693 4015654641032.174250906693 4026554641032.174250906693 4035664701055.391324138362 4046564701055.391324138362 4056654701055.391324138362 4065674731062.778614051647 4076574731062.778614051647 4085764731062.778614051647 4097564731062.778614051647 4106754731062.778614051647 4117654731062.778614051647 4125774791072.316808445408 4137574791072.316808445408 4147754791072.316808445408 4156664821080.3309413287764 4166674831091.075234880618 4176764831091.075234880618 4187664831091.075234880618 4196774861102.130696600507 4207674861102.130696600507 4217764861102.130696600507 4227774891111.726214476271

TRIPLETTYPE PARAMS:

INDEX: 1 ATOMS: C C N

PAIRS: CC CN CN UNIQUE: -1 TOTAL: 433

index I powers I equiv index I param index I parameter

00110030.37185085806

$101332-1.817798809255$

$203132-1.817798809255$

30145314.46325247754

40415314.46325247754

5015747.687688337064

6051747.687688337064

7016958.178218556836

8061958.178218556836

$9017116-1.084459976351$

$10071116-1.084459976351$

$11022137-57.73149130629$

$12023148-24.66551693906$

$13032148-24.66551693906$

$14024169-45.08978032921$ 
$15042169-45.08978032921$ $160251810-6.550702629822$ $170521810-6.550702629822$ $180262011-16.06540893858$ $190622011-16.06540893858$ 2002722120.4212407730846 2107222120.4212407730846 $220332413-25.98516421114$ $230342514-5.663823959139$ $240432514-5.663823959139$ 2503527154.778252988171 2605327154.778252988171 2703731171.234750857933 2807331171.234750857933 $290443318-22.65391174299$ 3004534195.939951952207 3105434195.939951952207 $320463620-7.200602394674$ $330643620-7.200602394674$ 3404738213.044562039281 3507438213.044562039281 3605540229.677050364126 3705641230.01558200423462 3806541230.01558200423462 3905743240.8800366405212 4007543240.8800366405212 $410664525-3.427333107474$ $420674626-0.9121122028856$ $430764626-0.9121122028856$ 4407748271.068616597332 45101492817.34720450641 46110492817.34720450641 $471025129-0.6742072022835$ $481205129-0.6742072022835$ $491035330-17.29896614531$ $501305330-17.29896614531$ 5110455311.597345611218 5214055311.597345611218 $531065933-7.632968075415$ $541605933-7.632968075415$ $551076134-15.03350054416$ $561706134-15.03350054416$ $571116335-64.60111264495$ 5811264360.7636034500298 5912164360.7636034500298 60114683825.35730745859 61141683825.35730745859 6211570394.204428032421 6315170394.204428032421 $641167240-17.88501445446$ $651617240-17.88501445446$ $661177441-3.71531126918$ $671717441-3.71531126918$ 68122764224.24064874225 $691237743-38.81262747898$ $701327743-38.81262747898$ 71124794426.71978430623 72142794426.71978430623 7312683462.512074202868 7416283462.512074202868 $751278547-20.19870420298$ $761728547-20.19870420298$ 77133874824.92330560532 7813590507.127564521808 7915390507.127564521808 $801369251-6.484460922084$ 
$811639251-6.484460922084$ $821379452-1.150558301527$ $831739452-1.150558301527$ 84145975410.92551901683 85154975410.92551901683 8614699552.734193794963 8716499552.734193794963 $8814710156-4.690193990408$ $8917410156-4.690193990408$ 90156104588.188873196073 91165104588.188873196073 $9215710659-1.223374232205$ $9317510659-1.223374232205$ 94166108604.111447408702 95167109611.833745743069 96176109611.833745743069 $9717711162-4.295721727977$ $9820111263-12.21371223359$ $9921011263-12.21371223359$ $10020211464-24.24288326835$ $10122011464-24.24288326835$ $10220311665-17.04049923445$ $10323011665-17.04049923445$ $10420411866-21.60113186946$ $10524011866-21.60113186946$ $10620512067-5.525366713802$ $10725012067-5.525366713802$ $10820612268-5.542486447022$ $10926012268-5.542486447022$ 110207124691.572344884426 111270124691.572344884426 1122111267046.25042145099 $11321212771-52.90280457685$ $11422112771-52.90280457685$ $11521312972-24.27237899709$ $11623112972-24.27237899709$ $11721713776-5.878622381099$ $11827113776-5.878622381099$ $11922213977-136.0344791422$ $12022314078-69.74212658738$ $12123214078-69.74212658738$ $12222414279-102.8616810025$ $12324214279-102.8616810025$ $12422514480-18.35270801346$ $12525214480-18.35270801346$ $12622614681-29.92159816146$ $12726214681-29.92159816146$ 128227148823.46672665588 129272148823.46672665588 $13023315083-52.63611056786$ $13123415184-18.96190889547$ $13224315184-18.96190889547$ $13323615586-7.167181612451$ $13426315586-7.167181612451$ $13524415988-51.95348493412$ 136245160897.088696365459 137254160897.088696365459 $13824616290-13.25502830741$ $13926416290-13.25502830741$ 140247164916.59190761337 141274164916.59190761337 142255166929.031254478348 $14325616793-3.429146786668$ $14426516793-3.429146786668$ $14526617195-4.913473516385$ $14626717296-2.106302120197$ 
$14727617296-2.106302120197$ 148277174972.03697415642 $14930217799-7.484465352672$ $15032017799-7.484465352672$ $151303179100-20.92854544823$ $152330179100-20.92854544823$ $153304181101-8.143787233863$ $154340181101-8.143787233863$ $155305183102-1.175328024497$ $156350183102-1.175328024497$ $157306185103-7.425658911164$ $158360185103-7.425658911164$ $159307187104-9.310154858941$ $160370187104-9.310154858941$ $161312190106-11.24380051493$ $162321190106-11.24380051493$ 16331319210726.66224944943 16433119210726.66224944943 $165316198110-20.57226849499$ $166361198110-20.57226849499$ 1673172001113.293517174014 1683712001113.293517174014 $169322202112-1.040993109521$ $170323203113-33.07537423236$ $171332203113-33.07537423236$ 1723252071152.741435546853 1733522071152.741435546853 $174326209116-1.923915253825$ $175362209116-1.923915253825$ $176327211117-11.25434208294$ $177372211117-11.25434208294$ 17833321311833.30614707519 $179334214119-12.22759629702$ $180343214119-12.22759629702$ 1813352161200.4764192218971 1823532161200.4764192218971 $183336218121-9.346147555454$ $184363218121-9.346147555454$ 1853372201223.427853070123 1863732201223.427853070123 $187344222123-10.99106348843$ 1883452231246.400383050949 1893542231246.400383050949 $190347227126-2.909213967953$ $191374227126-2.909213967953$ $192355229127-7.127010054035$ 1933562301285.563306955464 1943652301285.563306955464 $195357232129-0.3930474268317$ $196375232129-0.3930474268317$ 1973662341301.896707225139 1983672351311.286387408092 1993762351311.286387408092 $200377237132-2.155490453791$ 20140123813312.00349973191 20241023813312.00349973191 $203402240134-24.66984335256$ $204420240134-24.66984335256$ $205403242135-1.356046465979$ $206430242135-1.356046465979$ $207404244136-12.10486738289$ $208440244136-12.10486738289$ 2094052461373.343248503922 2104502461373.343248503922 $211406248138-1.193359831357$ $212460248138-1.193359831357$ 
2134072501392.351244241956 2144702501392.351244241956 21541125214010.08021772484 $216413255142-17.91148618007$ $217431255142-17.91148618007$ 2184142571433.7827436182 2194412571433.7827436182 $220417263146-3.560902502067$ $221471263146-3.560902502067$ $222422265147-101.6591062875$ $223423266148-20.89600656602$ $224432266148-20.89600656602$ $225424268149-56.49159479981$ $226442268149-56.49159479981$ 2274252701502.659890239815 2284522701502.659890239815 $229426272151-12.74786322393$ $230462272151-12.74786322393$ 2314272741524.881001967834 2324722741524.881001967834 $233433276153-27.25405602059$ $234434277154-6.478665330405$ $235443277154-6.478665330405$ 2364352791551.602878346898 2374532791551.602878346898 $238436281156-2.988613616345$ $239463281156-2.988613616345$ 2404372831570.6513731019246 2414732831570.6513731019246 $242444285158-19.53329689226$ 2434452861596.752408735442 2444542861596.752408735442 $245446288160-3.440832610766$ $246464288160-3.440832610766$ 2474472901613.961501723029 2484742901613.961501723029 2494552921626.061963468847 $250456293163-0.6808271844082$ $251465293163-0.6808271844082$ 2524572951640.8260363393579 2534752951640.8260363393579 $254466297165-1.560183023295$ $255467298166-0.5053751974196$ $256476298166-0.5053751974196$ 2574773001671.788813813714 25850130116823.65254958098 25951030116823.65254958098 $260502303169-1.858156466848$ $261520303169-1.858156466848$ 2625033051705.716960979918 2635303051705.716960979918 $264504307171-0.5668789526107$ $265540307171-0.5668789526107$ 26650530917211.09617651067 26755030917211.09617651067 $268506311173-0.2066088780419$ $269560311173-0.2066088780419$ $270507313174-1.116362304446$ $271570313174-1.116362304446$ 2725113151758.331952268165 27351231617629.38529306078 27452131617629.38529306078 2755133181775.456303481838 2765313181775.456303481838 2775143201789.708171075779 2785413201789.708171075779 
$279515322179-8.926476859206$ $280551322179-8.926476859206$ $281516324180-1.982581098977$ $282561324180-1.982581098977$ 2835173261816.523946623489 2845713261816.523946623489 $285522328182-0.155785616225$ 2865233291835.348373060457 2875323291835.348373060457 2885243311842.934990200645 2895423311842.934990200645 29052533318518.50169953862 29155233318518.50169953862 2925263351863.970111426117 2935623351863.970111426117 $294535342190-9.195533448794$ $295553342190-9.195533448794$ 2965363441911.873165049962 2975633441911.873165049962 2985373461924.581864075384 2995733461924.581864075384 $300544348193-0.1185814059709$ 30154534919411.99061494142 30255434919411.99061494142 3035463511954.16844623058 3045643511954.16844623058 3055473531961.534547123394 3065743531961.534547123394 $307555355197-7.529541201432$ 3085563561988.034440518241 3095653561988.034440518241 3105573581991.306545117679 3115753581991.306545117679 3125663602002.791074167173 3135673612011.985071016808 3145763612011.985071016808 3155773632020.1071353629458 3166013642030.01477165682629 3176103642030.01477165682629 $318602366204-11.33622727119$ $319620366204-11.33622727119$ $320603368205-2.471797982687$ $321630368205-2.471797982687$ $322604370206-3.40356689129$ $323640370206-3.40356689129$ 3246053722072.011441277205 3256503722072.011441277205 3266073762091.370268597925 3276703762091.370268597925 $328611378210-29.01237803778$ $329612379211-4.577121852352$ $330621379211-4.577121852352$ $331613381212-23.06774570316$ $332631381212-23.06774570316$ 3336143832133.21140067573 3346413832133.21140067573 3356163872154.311988607809 3366613872154.311988607809 3376173892161.261982804432 3386713892161.261982804432 $339622391217-40.71731127267$ $340623392218-8.738842564272$ $341632392218-8.738842564272$ $342624394219-18.18455212026$ $343642394219-18.18455212026$ 3446253962203.223716089432 
3456523962203.223716089432 $346626398221-3.636957701221$ $347662398221-3.636957701221$ 3486274002222.664053076343 3496724002222.664053076343 $350633402223-16.60273295363$ 3516354052252.974004216251 3526534052252.974004216251 3536364072261.80868728595 3546634072261.80868728595 3556374092273.20996366346 3566734092273.20996366346 $357644411228-4.990047629943$ 3586454122294.592408340112 3596544122294.592408340112 $360646414230-0.5303932746402$ $361664414230-0.5303932746402$ 3626474162312.353975079464 3636744162312.353975079464 3646554182326.324934567894 3656564192331.41559358914 3666654192331.41559358914 3676574212342.921015357347 3686754212342.921015357347 $369666423235-0.2625569352357$ 3706674242360.3460321848665 3716764242360.3460321848665 3726774262371.9937013485 $373701427238-4.205337449113$ $374710427238-4.205337449113$ $375702429239-1.712719159545$ $376720429239-1.712719159545$ $377703431240-5.977152434309$ $378730431240-5.977152434309$ $379704433241-0.8237378546468$ $380740433241-0.8237378546468$ 3817054352420.44156938238 3827504352420.44156938238 3837064372431.118551372542 3847604372431.118551372542 $385707439244-0.4457077554796$ $386770439244-0.4457077554796$ $387711441245-0.1800654895879$ $388712442246-7.552662566385$ $389721442246-7.552662566385$ $390713444247-0.1228546965711$ $391731444247-0.1228546965711$ $392714446248-9.241824652165$ $393741446248-9.241824652165$ $394715448249-0.6486673404523$ $395751448249-0.6486673404523$ $396716450250-2.797636735319$ $397761450250-2.797636735319$ 3987174522514.756465943045 3997714522514.756465943045 $400722454252-4.213145930906$ $401723455253-9.147092963273$ $402732455253-9.147092963273$ $403724457254-1.156989343976$ $404742457254-1.156989343976$ 4057254592551.95932576192 4067524592551.95932576192 4077264612562.918843240224 4087624612562.918843240224 $409733465258-0.9608610455649$ $410734466259-7.825301749954$ 
$411743466259-7.825301749954$ $412735468260-0.5630065928071$ $413753468260-0.5630065928071$ $414736470261-0.7188258600063$ $415763470261-0.7188258600063$ 4167374722623.263818475699 4177734722623.263818475699 4187444742630.9157176967279 4197454752640.9589175769118 4207544752640.9589175769118 4217464772653.097336133278 4227644772653.097336133278 4237474792660.1847545148248 4247744792660.1847545148248 4257564822681.820034366029 4267654822681.820034366029 4277574842691.268343364254 4287754842691.268343364254 4297664862701.773853885849 4307674872710.3304827205778 4317764872710.3304827205778 4327774892720.08457059733774

TRIPLETTYPE PARAMS: INDEX: 2 ATOMS: C C O

PAIRS: CC CO CO UNIQUE: -1 TOTAL: 418

index I powers I equiv index I param index I parameter

0011003.662866498329

$101211-34.73559766302$

$202111-34.73559766302$

$301332-11.27105224735$

$403132-11.27105224735$

$501453-26.73920349101$

$604153-26.73920349101$

$701574-0.002638687556425$

$805174-0.002638687556425$

9016954.958111085612

10061954.958111085612

110171161.053421870841

120711161.053421870841

$13022137-32.45662844173$

$14023148-31.15510507322$

$15032148-31.15510507322$

$16024169-25.09488923201$

$17042169-25.09488923201$

$180251810-12.51969709261$

$190521810-12.51969709261$

$200262011-12.65828117255$

$210622011-12.65828117255$

$220272212-2.082703023296$

$230722212-2.082703023296$

$240332413-14.89480189564$

$250342514-19.20121333704$ $260432514-19.20121333704$ 2703629161.411436874112 2806329161.411436874112 $290443318-18.33822372193$ $300453419-6.474238098099$ $310543419-6.474238098099$ $320463620-14.09538391294$ $330643620-14.09538391294$ $340473821-2.33771012617$ $350743821-2.33771012617$ $360554022-3.94121501126$ $370564123-0.787898387494$ 
$380654123-0.787898387494$ $390574324-1.454190163917$ $400754324-1.454190163917$ $410664525-9.819095415737$ $420674626-0.01472920278766$ $430764626-0.01472920278766$ $440774827-1.258597668667$ 45101492828.61712049231 46110492828.61712049231 4710251291.035241484731 4812051291.035241484731 4910353309.155179608605 5013053309.155179608605 $511045531-7.037753754903$ $521405531-7.037753754903$ 5310557322.101075906683 5415057322.101075906683 $551065933-7.021051235459$ $561605933-7.021051235459$ 5710761341.19578133021 5817061341.19578133021 $591116335-30.14484146512$ 6011264365.490553407982 6112164365.490553407982 $621136637-12.38924884773$ $631316637-12.38924884773$ $641167240-6.882432702686$ $651617240-6.882432702686$ 6611774413.213120712202 6717174413.213120712202 6812276423.201619076207 $691237743-14.65375574711$ $701327743-14.65375574711$ $711247944-3.149401638616$ $721427944-3.149401638616$ $731258145-1.374204660339$ $741528145-1.374204660339$ $751278547-0.7371784468162$ $761728547-0.7371784468162$ $771348849-5.59574595753$ $781438849-5.59574595753$ 79135905010.32862690541 80153905010.32862690541 8113794524.602869548015 8217394524.602869548015 8314699557.557474715733 8416499557.557474715733 $8514710156-0.1022222610536$ $8617410156-0.1022222610536$ 87155103578.51866736194 $8815610458-4.508757140867$ $8916510458-4.508757140867$ $9015710659-0.1930997060587$ $9117510659-0.1930997060587$ 92166108601.205379204481 $9316710961-2.543243994001$ $9417610961-2.543243994001$ 95177111620.4672135984211 $9620111263-87.28605983124$ $9721011263-87.28605983124$ 98202114646.673030112838 99220114646.673030112838 $10020311665-26.93681857316$ $10123011665-26.93681857316$ 1022041186614.41950453758 1032401186614.41950453758 
$10420512067-9.964394328308$ $10525012067-9.964394328308$ 106206122681.315045053521 107260122681.315045053521 $10820712469-4.213496670941$ $10927012469-4.213496670941$ $11021112670-103.004501722$ $11121212771-103.7965182833$ $11222112771-103.7965182833$ $11321413173-29.22166450697$ $11424113173-29.22166450697$ 115215133749.746916149345 116251133749.746916149345 $11721613575-0.06488201726377$ $11826113575-0.06488201726377$ $11922213977-18.47878950227$ $12022314078-26.29369025848$ $12123214078-26.29369025848$ 122224142790.525425163482 123242142790.525425163482 $12422514480-3.414077315792$ $12525214480-3.414077315792$ $12622614681-10.43128229008$ $12726214681-10.43128229008$ $12822714882-2.074251440073$ $12927214882-2.074251440073$ 1302331508349.37980723983 131234151847.592869234572 132243151847.592869234572 1332351538532.58688621003 1342531538532.58688621003 135236155866.529256415542 136263155866.529256415542 137237157875.822337467638 138273157875.822337467638 139244159884.524810550802 140245160898.264608963563 141254160898.264608963563 $14224616290-13.45094955261$ $14326416290-13.45094955261$ 144255166926.440604041865 145256167930.6495838000506 146265167930.6495838000506 147257169940.5987828206688 148275169940.5987828206688 $14926617195-13.0079704435$ 150267172960.280809603265 151276172960.280809603265 $15227717497-0.735310690612$ $15330217799-4.772501737817$ $15432017799-4.772501737817$ $155304181101-9.15907175486$ $156340181101-9.15907175486$ 1573051831022.290990997001 1583501831022.290990997001 $159306185103-6.012293252897$ $160360185103-6.012293252897$ $161312190106-0.9299037224205$ $162321190106-0.9299037224205$ 16331319210731.14770344129 16433119210731.14770344129 1653141941083.821460057355 1663411941083.821460057355 1673151961095.15707846007 1683511961095.15707846007 1693172001112.759472833027 
1703712001112.759472833027 $171323203113-0.5726454673069$ $172332203113-0.5726454673069$ $173324205114-6.448772218839$ $174342205114-6.448772218839$ 1753252071156.746557736671 1763522071156.746557736671 $177326209116-0.244551408538$ $178362209116-0.244551408538$ $179327211117-0.05424573676212$ $180372211117-0.05424573676212$ 18133321311840.55978052945 18233521612012.76979610828 18335321612012.76979610828 1843362181213.054145130597 1853632181213.054145130597 1863372201224.980336783944 1873732201224.980336783944 $188344222123-4.616540903041$ 1893452231240.5950908052486 1903542231240.5950908052486 1913462251252.86328781564 1923642251252.86328781564 $193347227126-1.577048081613$ $194374227126-1.577048081613$ $195355229127-0.1610172682857$ $196356230128-2.87474275701$ $197365230128-2.87474275701$ $198357232129-1.587624354266$ $199375232129-1.587624354266$ $200366234130-1.208562842893$ $201367235131-2.218575808192$ $202376235131-2.218575808192$ 2033772371320.4265187541962 $204401238133-4.830433392774$ $205410238133-4.830433392774$ $206402240134-7.634935463413$ $207420240134-7.634935463413$ 2084032421355.653286420934 2094302421355.653286420934 $210404244136-1.955463054506$ $211440244136-1.955463054506$ 2124052461374.583793082192 2134502461374.583793082192 $214407250139-0.5735657335086$ $215470250139-0.5735657335086$ $216412253141-1.136893033833$ $217421253141-1.136893033833$ 2184132551429.701617982982 2194312551429.701617982982 $220414257143-11.96864623951$ $221441257143-11.96864623951$ $222415259144-5.926013121313$ $223451259144-5.926013121313$ $224417263146-1.208276798888$ $225471263146-1.208276798888$ $226422265147-42.70828349516$ 22742326614811.65851488214 22843226614811.65851488214 $229424268149-21.81274850874$ $230442268149-21.81274850874$ 23142527015011.22972277905 23245227015011.22972277905 $233426272151-7.400371954443$ $234462272151-7.400371954443$ 2354272741521.824222173874 
2364722741521.824222173874 23743327615312.28877057385 2384362811562.426031098984 2394632811562.426031098984 2404372831571.486636585028 2414732831571.486636585028 $242444285158-13.40900534472$ 2434452861592.552731557014 2444542861592.552731557014 $245446288160-9.350344979111$ $246464288160-9.350344979111$ 2474472901610.8515379508977 2484742901610.8515379508977 $249455292162-8.962256653368$ $250457295164-0.4281303502464$ $251475295164-0.4281303502464$ $252466297165-6.270327146822$ 2534672981660.48135076012 2544762981660.48135076012 2554773001670.0522361146686 25650130116821.60881673991 25751030116821.60881673991 $258502303169-1.484365257964$ $259520303169-1.484365257964$ 2605033051709.022844072435 2615303051709.022844072435 $262504307171-0.7380252663573$ $263540307171-0.7380252663573$ 2645053091724.873861669017 2655503091724.873861669017 2665063111730.6224278390657 2675603111730.6224278390657 $268507313174-0.6840161949554$ $269570313174-0.6840161949554$ 27051131517542.05710937083 27151231617617.06522035273 27252131617617.06522035273 27351331817711.2993139571 27453131817711.2993139571 $275514320178-3.709508734604$ $276541320178-3.709508734604$ $277515322179-3.746087058736$ $278551322179-3.746087058736$ 2795163241807.801731269046 2805613241807.801731269046 $281517326181-0.6032139598269$ $282571326181-0.6032139598269$ $283522328182-0.960328965218$ 2845253331851.997039496681 2855523331851.997039496681 2865263351864.387249180888 2875623351864.387249180888 $288527337187-2.644886776899$ $289572337187-2.644886776899$ $290533339188-5.097002229334$ $291534340189-10.00332426566$ $292543340189-10.00332426566$ $293535342190-7.063611446377$ $294553342190-7.063611446377$ 2955363441914.789088448737 2965633441914.789088448737 2975443481930.595898871805 $298545349194-4.549533169909$ $299554349194-4.549533169909$ 3005463511953.354706855496 3015643511953.354706855496 
$302547353196-3.194367717587$ $303574353196-3.194367717587$ $304555355197-6.346129993952$ $305557358199-1.3286036633$ $306575358199-1.3286036633$ $307567361201-0.7841305051307$ $308576361201-0.7841305051307$ 3095773632020.8143060645347 $310601364203-10.95987787479$ $311610364203-10.95987787479$ 3126023662040.6933481445361 3136203662040.6933481445361 3146043702062.492580760959 3156403702062.492580760959 $316605372207-1.556794774667$ $317650372207-1.556794774667$ $318606374208-0.1910157293436$ $319660374208-0.1910157293436$ $320607376209-2.702435726795$ $321670376209-2.702435726795$ $322611378210-25.39005447176$ $323612379211-1.373421670377$ $324621379211-1.373421670377$ $325614383213-0.5741278116563$ $326641383213-0.5741278116563$ $327615385214-5.358940607249$ $328651385214-5.358940607249$ $329616387215-4.023069610138$ $330661387215-4.023069610138$ 33162339221811.94260805781 33263239221811.94260805781 3336243942193.211353015825 3346423942193.211353015825 3356253962205.106347188674 3366523962205.106347188674 $337626398221-0.3383563636217$ $338662398221-0.3383563636217$ $339627400222-1.400775491229$ $340672400222-1.400775491229$ 34163340222313.74067785192 3426344032247.835671536209 3436434032247.835671536209 3446354052255.497067835364 3456534052255.497067835364 3466364072260.5069448777286 3476634072260.5069448777286 3486374092274.089633640965 3496734092274.089633640965 3506444112282.8199507717 3516454122294.406643685756 3526544122294.406643685756 $353646414230-0.5283265628968$ $354664414230-0.5283265628968$ 3556474162310.5326572031855 3566744162310.5326572031855 3576554182322.086681837846 3586564192330.4011049755066 3596654192330.4011049755066 3606574212343.068730340678 3616754212343.068730340678 $362666423235-0.5457300810416$ $363667424236-0.03513979617056$ $364676424236-0.03513979617056$ 3656774262371.79441349236 $366701427238-0.5878902500104$ $367710427238-0.5878902500104$ 
3687024292390.8094536852883 3697204292390.8094536852883 3707034312401.832847443038 3717304312401.832847443038 3727044332412.925982309136 3737404332412.925982309136 3747054352421.972620505764 3757504352421.972620505764 3767064372431.017755119904 3777604372431.017755119904 3787074392440.175052384996 3797704392440.175052384996 $380712442246-2.362781940976$ $381721442246-2.362781940976$ 3827164502503.708302777878 3837614502503.708302777878 3847174522511.525868035812 3857714522511.525868035812 3867224542523.157785896754 $387723455253-0.1595992694516$ $388732455253-0.1595992694516$ 3897244572543.822479547466 3907424572543.822479547466 3917264612561.870432808596 3927624612561.870432808596 $393727463257-0.6690735368193$ $394772463257-0.6690735368193$ $395733465258-2.480855168807$ $396734466259-2.392106725487$ $397743466259-2.392106725487$ $398735468260-2.038096569632$ $399753468260-2.038096569632$ 4007364702611.380966709007 4017634702611.380966709007 4027374722621.535783108258 4037734722621.535783108258 4047444742631.066654679559 405745475264 -3.327619119845 $406754475264-3.327619119845$ $407747479266-1.571290643854$ $408774479266-1.571290643854$ $409755481267-2.251771413221$ $410756482268-1.555902840829$ $411765482268-1.555902840829$ 4127574842690.638254882966 4137754842690.638254882966 $414766486270-0.7520933772805$ $415767487271-0.7384752716855$ $416776487271-0.7384752716855$ 4177774892721.208609228651

TRIPLETTYPE PARAMS:

INDEX: 3 ATOMS: C N N

PAIRS: CN CN NN UNIQUE: -1 TOTAL: 427

index I powers I equiv index I param index I parameter

00110022.91108143871

11010022.91108143871

$201221-14.95446092698$

$310221-14.95446092698$

$401342-5.87918308356$

$510342-5.87918308356$

$601463-18.33547631195$

$710463-18.33547631195$

$801584-6.845316623963$

$910584-6.845316623963$ 
$10016105-15.83761341795$ $11106105-15.83761341795$ 1201712612.37326720417 1310712612.37326720417 $14021147-33.81970597002$ $15201147-33.81970597002$ $16022168-34.52463997808$ $17202168-34.52463997808$ $18023189-19.81935652809$ $19203189-19.81935652809$ $200242010-10.36679373019$ $212042010-10.36679373019$ 2202522115.458646314223 2320522115.458646314223 $240262412-2.946554353828$ $252062412-2.946554353828$ 2602726137.282202700529 2720726137.282202700529 $280312814-11.85694157462$ $293012814-11.85694157462$ $300323015-14.97588381964$ $313023015-14.97588381964$ $320333216-9.01440845152$ $333033216-9.01440845152$ $340343417-2.493021230088$ $353043417-2.493021230088$ $360363819-7.802573578812$ $373063819-7.802573578812$ 3803740207.838900541605 3930740207.838900541605 $400414221-25.32289975771$ $414014221-25.32289975771$ $420424422-17.11179204641$ $434024422-17.11179204641$ 4404346230.75138831798 4540346230.75138831798 4604448247.537661655896 4740448247.537661655896 48045502513.44677678425 49405502513.44677678425 5004652260.6589002403624 5140652260.6589002403624 5204754276.215533727445 5340754276.215533727445 5405360302.47600345217 5550360302.47600345217 5605564321.101391802878 5750564321.101391802878 $580566633-3.711030154575$ $595066633-3.711030154575$ 6005768343.543315210607 6150768343.543315210607 $620617035-23.02008372156$ $636017035-23.02008372156$ $640627236-7.10184461998$ $656027236-7.10184461998$ $660637437-7.583357495995$ $676037437-7.583357495995$ 6806476383.767611952425 6960476383.767611952425 7006578391.815372301865 7160578391.815372301865 7206680401.978074110893 7360680401.978074110893 7406782414.47321799156 7560782414.47321799156 
7607184424.03412159282 7770184424.03412159282 $780728643-3.200752186466$ $797028643-3.200752186466$ $800738844-2.172030695386$ $817038844-2.172030695386$ $820749045-5.723881768946$ $837049045-5.723881768946$ $840759246-2.397585016846$ $857059246-2.397585016846$ $860769447-2.896755312826$ $877069447-2.896755312826$ $880779648-0.9421874598157$ $897079648-0.9421874598157$ 90110984917.82661080614 $911119950-68.17736272124$ $9211310152-13.79025161997$ 93114102538.190752982018 $9411610455-19.84401782188$ $9512010657-32.79048764878$ $9621010657-32.79048764878$ $9712110858-16.59688267613$ $9821110858-16.59688267613$ $9912211059-54.61273895298$ $10021211059-54.61273895298$ 101123112603.143620922329 102213112603.143620922329 1031251166211.49716390847 1042151166211.49716390847 $10512611863-9.843634245705$ $10621611863-9.843634245705$ 1071271206414.32053005677 1082171206414.32053005677 109132126672.060370229349 110312126672.060370229349 1111331286845.83126607836 1123131286845.83126607836 1131341306912.11033273037 1143141306912.11033273037 1151351327024.15826361589 1163151327024.15826361589 $11713613471-9.338040087383$ $11831613471-9.338040087383$ 119137136721.454299584221 120317136721.454299584221 $12114013873-3.653303769656$ $12241013873-3.653303769656$ $12314114074-7.824934627989$ $12441114074-7.824934627989$ $12514314476-8.249532784488$ $12641314476-8.249532784488$ 1271441467710.47274309883 1284141467710.47274309883 $12914514878-2.380856143854$ $13041514878-2.380856143854$ 131146150791.220554791016 132416150791.220554791016 1331501548117.81188108573 1345101548117.81188108573 1351521588313.56943959263 1365121588313.56943959263 137156166870.8241849507995 138516166870.8241849507995 $13916017089-0.0865105093432$ $14061017089-0.0865105093432$ $14116117290-10.50707766369$ 
$14261117290-10.50707766369$ 143162174918.736919874935 144612174918.736919874935 1451641789317.99828870137 1466141789317.99828870137 1471651809410.82583961161 1486151809410.82583961161 1491661829510.63179031275 1506161829510.63179031275 151170186971.580298884292 152710186971.580298884292 $15317118898-3.830755319432$ $15471118898-3.830755319432$ 1551731921001.376116637473 1567131921001.376116637473 1571751961021.067093344007 1587151961021.067093344007 $159176198103-1.036548350268$ $160716198103-1.036548350268$ 1611772001040.1338224287437 1627172001040.1338224287437 $163220202105-0.6033000255209$ $164221203106-53.12609774127$ $165222204107-72.44759355164$ $166223205108-13.78966888876$ $167224206109-17.8659207381$ 16822520711033.14356791242 $169226208111-7.228223255076$ 17022720911225.58121996836 1712302101131.447579055902 1723202101131.447579055902 17323321611614.71408842892 17432321611614.71408842892 17523421811720.14733582802 17632421811720.14733582802 1772352201185.643134891146 1783252201185.643134891146 $179236222119-6.089847690434$ $180326222119-6.089847690434$ 1812372241204.635180307596 1823272241204.635180307596 1832402261212.487512149296 1844202261212.487512149296 $185241228122-17.36891730947$ $186421228122-17.36891730947$ $187242230123-32.95895109194$ $188422230123-32.95895109194$ 18924323212412.88715108319 19042323212412.88715108319 1912442341251.199715059629 1924242341251.199715059629 19324523612624.46174399938 19442523612624.46174399938 $195246238127-7.252816931414$ $196426238127-7.252816931414$ 19724724012814.67188386894 19842724012814.67188386894 1992512441304.006427053072 2005212441304.006427053072 2012522461312.335449042108 2025222461312.335449042108 2032532481322.536483804349 2045232481322.536483804349 $205254250133-0.1710737612374$ $206524250133-0.1710737612374$ $207255252134-7.597672587547$ 
$208525252134-7.597672587547$ $209256254135-6.557267350371$ $210526254135-6.557267350371$ $211257256136-1.363551784439$ $212527256136-1.363551784439$ $213260258137-0.7937722702682$ $214620258137-0.7937722702682$ $215261260138-26.59343930759$ $216621260138-26.59343930759$ $217262262139-12.39745158429$ $218622262139-12.39745158429$ $219263264140-6.098923443376$ $220623264140-6.098923443376$ 2212642661413.583491325394 2226242661413.583491325394 2232652681426.354204037967 2246252681426.354204037967 2252662701431.358225924246 2266262701431.358225924246 2272672721449.846786380689 2286272721449.846786380689 2292702741452.414346088583 2307202741452.414346088583 23127127614610.32878456181 23272127614610.32878456181 $233272278147-0.4607234282955$ $234722278147-0.4607234282955$ $235273280148-0.4197370042033$ $236723280148-0.4197370042033$ $237274282149-5.60951254642$ $238724282149-5.60951254642$ $239275284150-4.111182693885$ $240725284150-4.111182693885$ $241276286151-3.418783693977$ $242726286151-3.418783693977$ $243277288152-3.368560689245$ $244727288152-3.368560689245$ 2453302901532.598557991687 24633129115465.2918045029 24733329315651.66968339829 $248334294157-18.29632354242$ 2493352951581.503894469216 $250336296159-22.16511753804$ $251337297160-8.611146690281$ 25234029816113.13551656184 25343029816113.13551656184 25434230216312.43581869014 25543230216312.43581869014 $256343304164-20.38563997008$ $257433304164-20.38563997008$ $258344306165-0.3693457462183$ $259434306165-0.3693457462183$ $260345308166-25.50780802187$ $261435308166-25.50780802187$ $262346310167-9.794122131478$ $263436310167-9.794122131478$ $264347312168-9.567476441405$ $265437312168-9.567476441405$ 2663503141692.262978708849 2675303141692.262978708849 $268352318171-16.94041945043$ $269532318171-16.94041945043$ $270353320172-17.09805112751$ $271533320172-17.09805112751$ $272354322173-26.57465463295$ $273534322173-26.57465463295$ 
$274355324174-20.25984281836$ $275535324174-20.25984281836$ $276356326175-12.81819679298$ $277536326175-12.81819679298$ $278357328176-8.406133795519$ $279537328176-8.406133795519$ 2803603301773.309595058029 2816303301773.309595058029 2823623341799.938342588181 2836323341799.938342588181 2843633361801.557122572846 2856333361801.557122572846 28636433818112.59430272508 28763433818112.59430272508 2883653401824.488084610839 2896353401824.488084610839 2903663421834.960701625358 2916363421834.960701625358 $292367344184-2.297836117515$ $293637344184-2.297836117515$ $294370346185-2.63894058613$ $295730346185-2.63894058613$ 2963713481860.866173600734 2977313481860.866173600734 $298372350187-6.208691673803$ $299732350187-6.208691673803$ 3003733521883.138249849447 3017333521883.138249849447 $302374354189-2.45318356003$ $303734354189-2.45318356003$ $304376358191-2.711599045273$ $305736358191-2.711599045273$ $306377360192-1.388647785412$ $307737360192-1.388647785412$ 3084403621934.270074406232 $309442364195-19.88514625994$ 3104433651965.468873963625 $311444366197-9.591356786873$ 3124453671982.234590439188 $313446368199-13.78894402762$ 3144473692005.301248453174 3154503702012.843482508125 3165403702012.843482508125 $317453376204-13.48955421868$ $318543376204-13.48955421868$ $319454378205-8.910819095243$ $320544378205-8.910819095243$ $321455380206-17.06862151377$ $322545380206-17.06862151377$ $323456382207-8.115969541671$ $324546382207-8.115969541671$ $325457384208-6.423693361208$ $326547384208-6.423693361208$ $327460386209-0.05769248415557$ $328640386209-0.05769248415557$ $329461388210-3.705818418564$ $330641388210-3.705818418564$ $331462390211-7.251559697697$ $332642390211-7.251559697697$ 3334633922123.596307458489 3346433922123.596307458489 3354643942131.306578769274 3366443942131.306578769274 3374653962145.468932811807 3386453962145.468932811807 $339466398215-0.6210647298373$ 
$340646398215-0.6210647298373$ 3414674002166.249835785409 3426474002166.249835785409 $343470402217-0.9244075454687$ $344740402217-0.9244075454687$ 34547140421810.97308922229 34674140421810.97308922229 $347472406219-2.940246312572$ $348742406219-2.940246312572$ 3494734082203.991074057832 3507434082203.991074057832 $351474410221-3.619272828832$ $352744410221-3.619272828832$ $353476414223-2.485591882687$ $354746414223-2.485591882687$ $355477416224-2.561533441186$ $356747416224-2.561533441186$ 3575504182251.414893573623 $358551419226-8.37795819487$ $359552420227-12.95418349665$ $360553421228-14.01860951473$ $361554422229-10.77214744062$ $362555423230-10.46531590432$ $363556424231-5.619650160417$ $364557425232-4.682514463871$ 3655604262334.309822274468 3666504262334.309822274468 36756142823418.92039250231 36865142823418.92039250231 36956243023512.41520209885 37065243023512.41520209885 37156343223617.26152903706 37265343223617.26152903706 37356443423712.48876487929 37465443423712.48876487929 37556543623810.17472814288 37665543623810.17472814288 3775664382394.103797700242 3786564382394.103797700242 $379567440240-0.5431238839804$ $380657440240-0.5431238839804$ 3815714442421.437678016923 3827514442421.437678016923 $383572446243-0.8770264159339$ $384752446243-0.8770264159339$ 3855734482444.943411683749 3867534482444.943411683749 3875744502454.094700096943 3887544502454.094700096943 3895754522462.162507639376 3907554522462.162507639376 $391576454247-0.1248861253343$ $392756454247-0.1248861253343$ $393577456248-1.011274567456$ $394757456248-1.011274567456$ 3956604582494.455655406693 39666145925010.9167669114 3976624602516.681345686526 39866346125212.49996890782 3996644622539.131112049864 4006654632548.977217411892 4016664642553.276352687487 4026674652564.298646267462 4036704662572.882226632979 4047604662572.882226632979 40567146825812.31074143849 
40676146825812.31074143849 4076724702596.703480337219 4087624702596.703480337219 40967347226010.0621882665 41076347226010.0621882665 4116744742615.751008173838 4127644742615.751008173838 4136754762625.107939264477 4147654762625.107939264477 4156764782630.9831338589094 4167664782630.9831338589094 $417677480264-1.073555017816$ $418767480264-1.073555017816$ $419770482265-0.09425209530269$ 4207714832663.778400417609 4217724842670.505670863778 4227734852685.785703718098 4237744862692.920000008632 4247754872702.416577308221 $425776488271-0.1042657594153$ $426777489272-0.3211342627023$

TRIPLETTYPE PARAMS:

INDEX: 4 ATOMS: C N O

PAIRS: CN CO NO UNIQUE: -1 TOTAL: 429

index I powers I equiv index I param index I parameter

00110020.52415121344

$101211-5.222651813877$

$201322-3.286984225376$

$301433-11.0021840051$

$401655-13.93181226354$

$502177-21.82784285083$

$602288-21.21296727507$

$702399-17.127298845$

$80241010-9.383831672142$

$90251111-10.33582027918$

$100261212-12.22058863939$

$110271313-7.898106225045$

$120311414-12.53456217421$

$130321515-19.69627670986$

$140331616-17.23890331554$

$150341717-10.99668647339$

$160351818-2.557928651492$

$170361919-17.69601700433$

$180372020-5.851040707029$

$190412121-17.10164328186$

$200422222-13.08695640162$ $210432323-6.36119824923$

2204424241.611580791397 $230462626-6.302567636017$ $240472727-5.233327518112$ $250512828-3.034133563396$ $260522929-3.495177198388$ 2705431310.5425809879434 2805532327.85050193203 $290563333-2.435042994115$ 3005734341.334767372362 $310613535-3.26181687607$ $320623636-4.479499059816$ $330633737-2.957125115996$ $340643838-0.5967220920703$ 3506539390.6331819751916 $360664040-0.1874929114487$ $370674141-2.347671934464$ $380714242-9.798798730574$ 
3907243431.029305200705 $400734444-8.017416388211$ $410744545-0.1117133862006$ $420754646-0.8118006116263$ 4307647471.379015797382 $440774848-0.3249787897683$ 45101494927.63197484593 $461025050-12.559126889$ $471055353-2.02694317425$ $481065454-20.20903399993$ $491075555-7.698997711999$ 50110565619.67861214145 $511115757-79.41731119874$ 52112585838.8226544176 53114606024.60561489681 $541156161-2.856849620422$ $551166262-0.4785703410009$ 56117636314.37942505641 $571226666-13.8324471791$ $581236767-10.80119633368$ 5912468684.452233919261 6012569697.795469064115 $611267070-8.223130553624$ 6213072721.028700370622 $631317373-45.94536735382$ 64132747417.05456776433 6513375757.089233897928 $661347676-0.7672032532582$ 67135777712.2285093932 68136787827.07002332698 69137797932.39374001422 7014080806.988107696046 71141818112.08580663503 $721438383-13.25990889091$ $731448484-19.45692136425$ 7414686863.621672947684 7514787873.340466796247 $761508888-4.677594152336$ 7715391919.684926305754 781549292 -8.32098669865 7915593937.58645759003 80156949418.80368201735 81157959515.84579182579 $821619797-5.520369294963$ 8316298983.879954868425 841651011012.303735604591 851661021021.030567286332 $86167103103-0.3548367668497$ $87171105105-2.369484630123$ 881721061062.056029731476 891731071071.633937517164 $90174108108-2.341197626816$ $91175109109-4.766067404785$ 921761101103.023234616791 $93201112112-37.90897389118$ $94203114114-10.22512085357$ $95204115115-2.299017410928$ $96205116116-7.485078456207$ $97206117117-6.617163562143$ $98207118118-2.965886291005$ $99210119119-13.6572345465$ $100211120120-7.74735145852$ $101212121121-40.99942119223$ $102213122122-2.380977143712$ 10321512412417.47598735642 $104216125125-17.54198016899$ 
10522012712716.44243002856 $106221128128-33.86472381819$ 1072221291293.967010692821 1082241311313.655749487802 $109225132132-4.497519826835$ $110226133133-21.11870342034$ $111227134134-11.52276492184$ 1122301351359.67940084736 11323113613634.13911908358 11423313813836.95703679492 11523413913920.76274158339 11623514014034.60814405465 $117236141141-14.92432387565$ $118237142142-1.697741291614$ 1192401431436.420850066028 $120242145145-0.1618535525461$ 12124314614620.72918075866 12224414714713.7204798945 12324514814811.44827403155 $124246149149-10.67461742712$ $125247150150-5.446856098539$ $126250151151-5.283634924523$ 1272511521528.073248627487 $128252153153-1.352265675304$ 12925315415422.79548891573 13025415515511.86864653527 13125515615625.44991195314 1322571581585.373954179472 $133260159159-8.784258411881$ $134261160160-14.7710502199$ $135262161161-15.42343986213$ $136263162162-5.718847546558$ $137264163163-4.515081213853$ $138266165165-2.196079936788$ $139267166166-3.781634744187$ $140270167167-4.852319975539$ $141271168168-13.9969665086$ $142272169169-1.701565274246$ $143273170170-6.341992283288$ 1442741711711.262633433711 1452751721722.970665731003 1462761731732.862000553973 1472771741741.052394896482 1483011751750.7080824743281 $149302176176-13.05107178146$ $150303177177-1.422833644153$ 1513041781782.085330685429 1523051791792.645251470104 $153306180180-11.51134308124$ $154307181181-4.336345758271$ $155310182182-24.22287389876$ $156311183183-59.24606089906$ $157312184184-23.93804873513$ 1583131851859.71870973189 $159314186186-2.300424524505$ $160316188188-2.255366268374$ 1613171891899.376242734483 $162320190190-3.795369574698$ $163322192192-5.95991026083$ 16432319319312.97883000453 1653241941949.970559851676 16632519519518.10566792967 $167326196196-1.293709338707$ 1683271971973.875413103306 $169330198198-15.40332693812$ $170331199199-9.074987060468$ 
17133320120123.26041180091 $172334202202-10.45975838333$ 1733352032034.727919072141 17433620420415.96329951539 17533720520519.25273758005 1763402062062.177837446409 1773412072070.993253885282 $178343209209-12.23472181308$ $179344210210-14.02660067204$ $180345211211-5.615952573829$ 1813462122121.313829905124 $182350214214-9.992534778515$ $183352216216-7.834714207621$ $184354218218-16.46205420491$ $185355219219-7.526472585574$ 1863562202207.574371722774 1873572212214.764184906611 $188360222222-1.545432032224$ $189361223223-1.898288654989$ 1903632252250.4258392336015 $191364226226-3.106435333884$ $192365227227-0.406277712004$ $193366228228-1.439685177308$ $194367229229-0.6571842450132$ 1953702302301.097520474603 1963712312318.301583353616 1973722322323.77165282628 1983732332333.039935456741 $199374234234-3.731098853217$ $200375235235-7.042022330906$ 2013762362360.6962524339992 $202377237237-2.415096321578$ $203401238238-8.133482094004$ $204402239239-11.92673412292$ $205404241241-1.530737239993$ 2064052422420.997201913785 $207406243243-5.30934417484$ $208407244244-4.496801827387$ 2094102452456.594690036776 21041124624611.69318500359 211412247247 -1.900236232136 2124142492491.298143700402 $213415250250-2.06415086718$ $214416251251-9.965743262322$ $215417252252-6.552684148534$ $216420253253-12.17604149813$ $217421254254-15.60107000783$ $218422255255-43.3689113432$ 2194232562560.9434148692208 $220424257257-10.93022873675$ 2214252582580.1703589318976 $222426259259-17.94549206583$ $223427260260-10.92294205149$ 2244302612614.493991891573 2254312622628.464452658571 2264332642643.301189983248 2274342652652.749322035411 $228436267267-11.90785568589$ $229437268268-7.006728159094$ $230440269269-6.370723960556$ $231441270270-3.297140779494$ $232442271271-19.86228127509$ 2334432722728.700295166485 $234444273273-0.6722214617041$ 2354452742743.09220848874 $236446275275-10.63938845253$ 
$237447276276-5.151339357781$ 2384502772776.142577882924 2394512782783.937815605201 24045227927912.76965606438 2414532802804.265436852606 2424542812815.441196592291 2434552822821.983508915561 $244456283283-1.849810029129$ $245457284284-0.6185928116874$ $246460285285-5.903422391676$ 2474612862862.381432360829 $248462287287-11.49226553679$ 2494632882882.731502780946 $250464289289-5.148537504437$ $251466291291-3.82856277152$ $252467292292-3.287868624015$ $253470293293-0.1496968134966$ $254471294294-2.476922518749$ 2554722952952.573844056424 $256473296296-1.985648134591$ 2574742972970.587138273515 2584762992990.776327283605 $259477300300-0.02623532292504$ 2605013013010.5911266413704 $261502302302-4.917542198792$ 2625043043044.522289808448 2635053053051.604525231212 $264506306306-3.421813645774$ $265507307307-3.032421054616$ 2665103083081.6212112925 2675133113114.376005891014 $268514312312-7.636248622244$ $269515313313-9.425663698052$ $270516314314-4.424643105143$ $271517315315-1.58851765231$ $272520316316-8.41355888545$ $273521317317-12.43101309741$ $274522318318-10.69753709972$ $275523319319-4.217503252271$ 2765243203203.200227548367 2775253213211.479350178608 $278527323323-1.502268482851$ $279530324324-0.2681726582813$ 2805323263261.339209090247 $281533327327-3.662452429408$ $282534328328-15.16014699625$ $283535329329-13.39833054069$ 2845363303301.70793118459 2855373313311.903834923331 2865403323323.481755268739 $287541333333-2.174461382396$ 2885423343343.908453593873 $289543335335-10.24595653078$ $290544336336-6.304165723611$ $291545337337-8.050119855415$ $292547339339-2.403182214444$ 2935503403405.418663683595 29455134134122.3478974539 2955523423425.468898488278 2965533433434.291756743127 $297554344344-10.23357652596$ $298555345345-8.683098134121$ $299556346346-0.9233780191209$ $300557347347-1.674278084578$ 3015603483486.41781730104 30256134934914.48017317343 
30356235035010.40154168666 3045633513517.462791139553 3055643523521.050742750793 $306565353353-0.375514040689$ $307566354354-1.082043186264$ $308567355355-1.12074864787$ 3095703563564.851472022338 31057135735716.42254016111 3115723583585.283867790851 3125733593594.444659642475 $313574360360-3.718824306839$ $314575361361-5.729673262438$ $315576362362-2.273481064819$ $316577363363-2.974231569562$ $317601364364-9.282648770981$ $318602365365-2.06271398514$ 3196043673674.593676238981 3206053683683.512579110126 3216063693691.425705700242 3226073703700.2238486009132 $323610371371-5.19966919208$ $324611372372-30.16200775494$ $325612373373-9.146662365153$ $326613374374-7.146157484578$ 32761437537512.62987213317 3286153763766.829627121979 $329617378378-0.5478479702762$ $330620379379-9.176541394429$ $331621380380-18.12667951508$ $332622381381-16.42942744302$ 3336243833831.045940525473 3346253843845.6120312234 $335626385385-1.67328355695$ $336630387387-7.959782878925$ $337631388388-17.06535537458$ $338632389389-11.6240429032$ 3396343913915.337290597897 3406353923926.594701977066 $341636393393-4.052209081947$ $342637394394-0.4153470730729$ $343640395395-5.553836663765$ 3446413963968.284127245967 $345642397397-8.285686870096$ 34664339839813.68552820007 3476443993990.3671373570351 3486454004008.274067679751 $349646401401-1.923724103225$ 3506474024021.494612876139 $351650403403-3.800097914651$ $352651404404-9.491468634627$ $353652405405-3.118105224316$ 3546544074071.594550469102 3556554084081.199672621425 356656409409 -2.617559306869 $357657410410-0.3166031749167$ $358660411411-5.760713797601$ $359662413413-8.168086673573$ 3606634144141.532136834672 $361664415415-4.31001960623$ $362665416416-0.0450970735591$ $363666417417-2.145275454021$ $364667418418-0.9228428962465$ $365670419419-5.611301017998$ $366671420420-11.7854768959$ $367672421421-7.750201937006$ $368673422422-6.701900195598$ 
$369674423423-3.864035524659$ $370675424424-3.247842444099$ $371676425425-2.364664436148$ $372677426426-1.058336435189$ $373701427427-5.214237129615$ $374702428428-0.07955379752538$ $375703429429-4.02656483303$ $376704430430-1.432062379068$ 3777064324320.2192734921456 3787074334330.06062478030281 3797104344347.318868382236 $380711435435-5.934891639676$ 38171243643615.37810468028 3827144384383.742071681306 $383715439439-5.025554871396$ 3847164404403.18207813091 3857204424422.569313097871 3867214434435.80604355915 3877224444447.75940752582 3887234454454.788023114556 3897244464461.228590498123 3907254474474.617986839279 3917264484483.561826354401 3927274494492.187566189033 3937304504508.587979901857 39473145145112.04758699153 39573245245217.66173657794 3967334534537.970085368565 3977344544542.204642683317 $398735455455-2.421733669462$ 3997364564563.936358471776 4007374574570.7827827146742 4017424604600.9427696243138 $402743461461-3.56136913405$ $403744462462-4.634844791457$ $404745463463-2.351994192427$ 4057464644640.61433065087 $406747465465-0.4827443262564$ 4077504664660.290887032017 $408753469469-4.718869596269$ $409754470470-5.964282773565$ $410755471471-7.274677973334$ $411756472472-0.8352665284795$ $412757473473-1.961362245829$ $413760474474-2.316021301668$ $414761475475-7.598368021683$ $415762476476-3.406147668353$ $416763477477-6.987546956564$ $417764478478-4.272818649733$ $418765479479-4.785775687548$ $419766480480-1.352450917926$ $420767481481-1.712684809433$ 4217704824822.041645399287 4227714834833.779481000938 4237724844842.467447227876 $424773485485-0.216705206108$ $425774486486-1.977312285195$ $426775487487-3.485861133627$ $427776488488-1.001581837218$ $428777489489-1.235302820719$

TRIPLETTYPE PARAMS:

INDEX: 5 ATOMS: C O O

PAIRS: CO CO OO UNIQUE: -1 TOTAL: 420 index I powers I equiv index I param index I parameter 
00110015.32830427042 11010015.32830427042 2012210.1780156719943 3102210.1780156719943 4013426.209932715515 5103426.209932715515 $601463-3.090190129675$ $710463-3.090190129675$ $801584-0.7503375987663$ $910584-0.7503375987663$ $10016105-6.108395336668$ $11106105-6.108395336668$ $12021147-6.039117652925$ $13201147-6.039117652925$ $14022168-23.00692558683$ $15202168-23.00692558683$ $16023189-4.405053169231$ $17203189-4.405053169231$ $180242010-5.639965759348$ $192042010-5.639965759348$ 20025221114.01096829739 21205221114.01096829739 22026241211.51232598253 23206241211.51232598253 2402726136.175253794837 2520726136.175253794837 2603128145.954469347731 2730128145.954469347731 $280323015-19.88591458791$ $293023015-19.88591458791$ $300333216-2.245730977545$ $313033216-2.245730977545$ $320343417-8.163740484129$ $333043417-8.163740484129$ 3403536183.913535221407 3530536183.913535221407 3603638191.279134610833 3730638191.279134610833 3803740202.319959434969 3930740202.319959434969 $400414221-5.467643981901$ $414014221-5.467643981901$ $420424422-24.92564180858$ 434024422 -24.92564180858 $440434623-14.62163189817$ $454034623-14.62163189817$ $460444824-12.21053404327$ $474044824-12.21053404327$ 4804550251.968992327534 4940550251.968992327534 5004652267.710821312542 5140652267.710821312542 5204754275.487037211902 5340754275.487037211902 $540525829-6.496422101204$ $555025829-6.496422101204$ $560536030-2.27021789928$ $575036030-2.27021789928$ $580576834-0.6943889687029$ $595076834-0.6943889687029$ 6006170350.2745286568417 6160170350.2745286568417 $620627236-6.915191861788$ $636027236-6.915191861788$ $640637437-1.824711457797$ $656037437-1.824711457797$ 
$660647638-4.513083460707$ $676047638-4.513083460707$ 6806578392.618465442417 6960578392.618465442417 7006782412.902406515039 7160782412.902406515039 $720718442-0.006692401111586$ $737018442-0.006692401111586$ $740728643-4.174674342201$ $757028643-4.174674342201$ $760738844-0.12279165103$ $777038844-0.12279165103$ $780749045-2.335128368711$ $797049045-2.335128368711$ 8007592461.89713709987 8170592461.89713709987 $820769447-1.727989327203$ $837069447-1.727989327203$ 8407796480.135059269398 8570796480.135059269398 86110984939.18501911115 $871119950-31.91197530057$ $8811410253-9.184865123404$ 891151035427.69887823371 $9012010657-32.22463243625$ $9121010657-32.22463243625$ $9212211059-21.26693518387$ $9321211059-21.26693518387$ 941231126036.0348843046 952131126036.0348843046 $9612611863-18.03922531391$ $9721611863-18.03922531391$ $9813012265-2.077044824601$ $9931012265-2.077044824601$ $10013212667-23.83606946767$ $10131212667-23.83606946767$ 1021331286814.13815578526 1033131286814.13815578526 104135132707.464614060887 105315132707.464614060887 $10613613471-5.242381582044$ $10731613471-5.242381582044$ $10813713672-6.976063990459$ $10931713672-6.976063990459$ $11014013873-15.68718256669$ $11141013873-15.68718256669$ $11214214275-11.48086433172$ $11341214275-11.48086433172$ 114144146774.49066228175 115414146774.49066228175 $11614514878-22.26983508575$ $11741514878-22.26983508575$ $11814615079-8.470247227965$ $11941615079-8.470247227965$ $12014715280-8.705844203455$ $12141715280-8.705844203455$ 122150154816.136627796801 123510154816.136627796801 124151156822.033412652294 125511156822.033412652294 126153160840.9007183336099 127513160840.9007183336099 128154162858.394042829191 129514162858.394042829191 $13015616687-0.2318915448268$ $13151616687-0.2318915448268$ 
132157168880.2072465039239 133517168880.2072465039239 $13416017089-8.252856479302$ $13561017089-8.252856479302$ $13616117290-15.35292398544$ $13761117290-15.35292398544$ 138163176923.083379598551 139613176923.083379598551 1401641789311.31519171807 1416141789311.31519171807 $14216518094-0.8001923772247$ $14361518094-0.8001923772247$ 144166182950.8552255259678 145616182950.8552255259678 146167184961.301097442978 147617184961.301097442978 148170186971.992208767544 149710186971.992208767544 $15017219099-5.39447161233$ $15171219099-5.39447161233$ 1521731921000.9791797294987 1537131921000.9791797294987 1541751961026.556158307252 1557151961026.556158307252 $156176198103-0.2302478986989$ $157716198103-0.2302478986989$ 1581772001043.10267014308 1597172001043.10267014308 1602202021051.311861148118 $161222204107-71.46182866651$ $162224206109-27.79409142867$ 16322520711042.87274764403 16422620811128.14639840608 16522720911217.88464012283 $166230210113-10.2491933465$ $167320210113-10.2491933465$ $168231212114-3.407246075149$ $169321212114-3.407246075149$ $170232214115-22.92606232733$ $171322214115-22.92606232733$ 17223321611612.39320687562 17332321611612.39320687562 $174236222119-2.76225782427$ $175326222119-2.76225782427$ 1762372241200.2930920430229 1773272241200.2930920430229 1782412281229.008775365893 1794212281229.008775365893 $180242230123-48.59673638123$ $181422230123-48.59673638123$ $182243232124-17.68642587684$ $183423232124-17.68642587684$ $184244234125-19.90136263398$ $185424234125-19.90136263398$ 18624523612610.95562525882 18742523612610.95562525882 18824623812719.87649413094 18942623812719.87649413094 19024724012810.97211425591 19142724012810.97211425591 1922502421291.210474337375 1935202421291.210474337375 $194251244130-0.6968481412277$ $195521244130-0.6968481412277$ 1962522461317.604227491153 1975222461317.604227491153 
1982532481324.620576273974 1995232481324.620576273974 20025425013315.42518270143 20152425013315.42518270143 $202255252134-2.702971398863$ $203525252134-2.702971398863$ 2042562541350.6668729111182 2055262541350.6668729111182 $206257256136-3.056398224335$ $207527256136-3.056398224335$ 2082612601389.65489271389 2096212601389.65489271389 $210262262139-11.45090200958$ $211622262139-11.45090200958$ 2122632641401.673797193861 2136232641401.673797193861 $214264266141-6.357543061837$ $215624266141-6.357543061837$ 2162652681425.802365066186 2176252681425.802365066186 2182662701431.923623267039 2196262701431.923623267039 2202672721444.575079921305 2216272721444.575079921305 2222702741452.605439883517 2237202741452.605439883517 $224271276146-2.274674207851$ $225721276146-2.274674207851$ 2262722781471.70998932914 2277222781471.70998932914 $228273280148-1.165479170089$ $229723280148-1.165479170089$ 2302742821492.227782673796 2317242821492.227782673796 $232276286151-2.209345839178$ $233726286151-2.209345839178$ $234277288152-1.183609566135$ $235727288152-1.183609566135$ 2363312911541.823903921988 2373322921556.221565461351 23833429415725.27517832677 $239335295158-14.04383588954$ $240337297160-9.940250948605$ 2413402981612.995916740596 2424302981612.995916740596 2433423021634.959233495153 2444323021634.959233495153 $245343304164-10.22650884064$ $246433304164-10.22650884064$ 24734430616514.67668300993 24843430616514.67668300993 $249345308166-15.27715653525$ $250435308166-15.27715653525$ 2513463101672.881943776599 2524363101672.881943776599 $253347312168-5.521332607424$ $254437312168-5.521332607424$ 25535031416912.22927623059 25653031416912.22927623059 $257351316170-0.2749570608778$ $258531316170-0.2749570608778$ 25935231817120.22864349954 26053231817120.22864349954 $261353320172-10.31363616055$ $262533320172-10.31363616055$ 26335432217320.59209401675 
26453432217320.59209401675 $265355324174-10.07514945372$ $266535324174-10.07514945372$ 2673563261753.766995078367 2685363261753.766995078367 $269360330177-5.510246165533$ $270630330177-5.510246165533$ $271362334179-1.541469773801$ $272632334179-1.541469773801$ 2733633361805.980320130574 2746333361805.980320130574 2753643381818.501534477782 2766343381818.501534477782 2773653401821.410724451833 2786353401821.410724451833 2793663421832.005995172989 2806363421832.005995172989 2813673441840.9211656142061 2826373441840.9211656142061 2833703461852.076857216732 2847303461852.076857216732 2853713481862.874833321532 2867313481862.874833321532 $287372350187-1.232520043893$ $288732350187-1.232520043893$ $289373352188-4.840149474876$ $290733352188-4.840149474876$ 2913743541891.224672453932 2927343541891.224672453932 $293375356190-0.5945593833283$ $294735356190-0.5945593833283$ 2953763581910.01608287260318 2967363581910.01608287260318 2973773601922.232639529868 2987373601922.232639529868 29944036219310.48737987309 $300441363194-5.501554223867$ $301442364195-11.91725825556$ $302443365196-24.34271374716$ $303445367198-0.1402449483422$ 30444636819917.34832963239 3054473692006.890668196165 $306450370201-1.618271498228$ $307540370201-1.618271498228$ 30845137220211.65403143275 30954137220211.65403143275 3104533762042.566640085961 3115433762042.566640085961 3124543782058.082209425932 3135443782058.082209425932 $314455380206-2.055667114064$ $315545380206-2.055667114064$ 3164563822070.91630413959 3175463822070.91630413959 $318457384208-1.773161191334$ $319547384208-1.773161191334$ $320460386209-0.5937431505903$ $321640386209-0.5937431505903$ 3224613882107.277992785704 3236413882107.277992785704 $324462390211-7.333476862247$ $325642390211-7.333476862247$ $326463392212-0.3509717167993$ $327643392212-0.3509717167993$ $328464394213-2.297319794598$ $329644394213-2.297319794598$ 
3304653962141.917795018354 3316453962141.917795018354 3324663982152.725546810962 3336463982152.725546810962 3344674002162.999876992902 3356474002162.999876992902 $336470402217-0.6886686570965$ $337740402217-0.6886686570965$ 3384714042181.616154577614 3397414042181.616154577614 $340472406219-3.985261749116$ $341742406219-3.985261749116$ $342473408220-3.43283797549$ $343743408220-3.43283797549$ $344474410221-1.700107673772$ $345744410221-1.700107673772$ $346475412222-0.7722523232571$ $347745412222-0.7722523232571$ $348476414223-1.753372679029$ $349746414223-1.753372679029$ 3504774162240.1352861585156 3517474162240.1352861585156 35255141922610.67233517264 $353552420227-5.850587312903$ $354553421228-5.801363245896$ $355554422229-2.466189209095$ $356555423230-5.781977420828$ $357556424231-0.8598733223364$ 3585574252323.531685795616 $359560426233-6.414073692991$ $360650426233-6.414073692991$ 3615614282345.09668647004 3626514282345.09668647004 $363562430235-6.50019178889$ $364652430235-6.50019178889$ 3655634322363.639338665985 3666534322363.639338665985 3675644342371.361708237918 3686544342371.361708237918 $369566438239-0.5171820655269$ $370656438239-0.5171820655269$ $371567440240-0.7742642563097$ $372657440240-0.7742642563097$ 3735704422410.7843150572205 3747504422410.7843150572205 3755714442422.319647557349 3767514442422.319647557349 $377572446243-4.553331089628$ $378752446243-4.553331089628$ $379573448244-7.414908580065$ $380753448244-7.414908580065$ $381574450245-5.345257624019$ $382754450245-5.345257624019$ $383575452246-4.018622494075$ $384755452246-4.018622494075$ $385576454247-2.127101456825$ $386756454247-2.127101456825$ 3875774562481.548975578545 3887574562481.548975578545 3896604582491.243805713278 $390661459250-1.447313567875$ 3916624602511.079034111542 392663461252 -3.590725145889 $393664462253-0.401440611692$ $394665463254-3.492275947758$ $395666464255-0.4856501330693$ 
$396667465256-0.1588390549759$ 3976704662572.896862081382 3987604662572.896862081382 $399671468258-6.288579795645$ $400761468258-6.288579795645$ 4016724702593.999529835303 4027624702593.999529835303 $403673472260-6.279200717798$ $404763472260-6.279200717798$ 4056744742612.073575091673 4067644742612.073575091673 $407675476262-3.609059101393$ $408765476262-3.609059101393$ $409676478263-0.4524875555728$ $410766478263-0.4524875555728$ $411677480264-1.246280907186$ $412767480264-1.246280907186$ 4137704822652.364547407126 $414771483266-1.644002405141$ $415773485268-6.138317072772$ $416774486269-3.14497849812$ $417775487270-3.399093364647$ $418776488271-2.161948280212$ 4197774892720.4689130755337

TRIPLETTYPE PARAMS:

INDEX: 6 ATOMS: N N N

PAIRS: NN NN NN UNIQUE: -1 TOTAL: 387

index I powers I equiv index I param index I parameter

$001231-28.99652971492$

$110231-28.99652971492$

$202131-28.99652971492$

$320131-28.99652971492$

$412031-28.99652971492$

$521031-28.99652971492$

60139213.86714738456

71039213.86714738456

80319213.86714738456

93019213.86714738456

101309213.86714738456

113109213.86714738456

$12014153-22.49150590629$

$13104153-22.49150590629$

$14041153-22.49150590629$

$15401153-22.49150590629$

$16140153-22.49150590629$

$17410153-22.49150590629$ 180152145.475593320794

191052145.475593320794 200512145.475593320794 215012145.475593320794 221502145.475593320794 235102145.475593320794 $24016275-33.91714123277$ $25106275-33.91714123277$ $26061275-33.91714123277$ $27601275-33.91714123277$ $28160275-33.91714123277$ $29610275-33.91714123277$ 300173365.832750036275 311073365.832750036275 320713365.832750036275 337013365.832750036275 341703365.832750036275 357103365.832750036275 
$36022397-25.3015701135$ $37202397-25.3015701135$ $38220397-25.3015701135$ $39023428-35.47070018498$ $40203428-35.47070018498$ $41032428-35.47070018498$ $42302428-35.47070018498$ $43230428-35.47070018498$ $44320428-35.47070018498$ $45024489-22.57193047211$ $46204489-22.57193047211$ $47042489-22.57193047211$ $48402489-22.57193047211$ $49240489-22.57193047211$ $50420489-22.57193047211$ $510255410-11.55808847664$ $522055410-11.55808847664$ $530525410-11.55808847664$ $545025410-11.55808847664$ $552505410-11.55808847664$ $565205410-11.55808847664$ 5702660115.148173729833 5820660115.148173729833 5906260115.148173729833 6060260115.148173729833 6126060115.148173729833 6262060115.148173729833 6302766120.4402244585785 6420766120.4402244585785 6507266120.4402244585785 6670266120.4402244585785 6727066120.4402244585785 6872066120.4402244585785 $690347514-22.79807516446$ $703047514-22.79807516446$ $710437514-22.79807516446$ $724037514-22.79807516446$ $733407514-22.79807516446$ $744307514-22.79807516446$ 7503581153.596041593721 7630581153.596041593721 7705381153.596041593721 7850381153.596041593721 7935081153.596041593721 8053081153.596041593721 $810368716-13.77010753683$ $823068716-13.77010753683$ $830638716-13.77010753683$ $846038716-13.77010753683$ $853608716-13.77010753683$ $866308716-13.77010753683$ 8704499185.584979924762 8840499185.584979924762 8944099185.584979924762 $9004510219-4.06491055981$ $9140510219-4.06491055981$ $9205410219-4.06491055981$ $9350410219-4.06491055981$ $9445010219-4.06491055981$ $9554010219-4.06491055981$ 96046108202.442298862357 97406108202.442298862357 98064108202.442298862357 99604108202.442298862357 100460108202.442298862357 101640108202.442298862357 
$10204711421-11.62590996234$ $10340711421-11.62590996234$ $10407411421-11.62590996234$ $10570411421-11.62590996234$ $10647011421-11.62590996234$ $10774011421-11.62590996234$ 108055120229.248275229723 109505120229.248275229723 110550120229.248275229723 $11105612323-5.032640176189$ $11250612323-5.032640176189$ $11306512323-5.032640176189$ $11460512323-5.032640176189$ $11556012323-5.032640176189$ $11665012323-5.032640176189$ $11706613525-1.896117955273$ $11860613525-1.896117955273$ $11966013525-1.896117955273$ $12006713826-3.028447284932$ $12160713826-3.028447284932$ $12207613826-3.028447284932$ $12370613826-3.028447284932$ $12467013826-3.028447284932$ $12576013826-3.028447284932$ 126077144271.651645710937 127707144271.651645710937 128770144271.651645710937 $12911114728-149.0007858005$ $13011214829-29.2353910329$ $13112114829-29.2353910329$ $13221114829-29.2353910329$ $13311616033-61.31308194486$ $13416116033-61.31308194486$ $13561116033-61.31308194486$ 1361231693636.25874169196 1372131693636.25874169196 1381321693636.25874169196 1393121693636.25874169196 1402311693636.25874169196 1413211693636.25874169196 $14212417537-22.03443077593$ $14321417537-22.03443077593$ $14414217537-22.03443077593$ $14541217537-22.03443077593$ $14624117537-22.03443077593$ $14742117537-22.03443077593$ 148125181386.357185911384 149215181386.357185911384 150152181386.357185911384 151512181386.357185911384 152251181386.357185911384 153521181386.357185911384 1541271934010.67568730652 1552171934010.67568730652 1561721934010.67568730652 1577121934010.67568730652 1582711934010.67568730652 1597211934010.67568730652 1601331994124.79665493155 1613131994124.79665493155 1623311994124.79665493155 $16313420242-4.643901211304$ $16431420242-4.643901211304$ $16514320242-4.643901211304$ $16641320242-4.643901211304$ $16734120242-4.643901211304$ 
$16843120242-4.643901211304$ $16913722045-4.16503851896$ $17031722045-4.16503851896$ $17117322045-4.16503851896$ $17271322045-4.16503851896$ $17337122045-4.16503851896$ $17473122045-4.16503851896$ 175146235485.830826073539 176416235485.830826073539 177164235485.830826073539 178614235485.830826073539 179461235485.830826073539 180641235485.830826073539 $18114724149-14.69664830735$ $18241724149-14.69664830735$ $18317424149-14.69664830735$ $18471424149-14.69664830735$ $18547124149-14.69664830735$ $18674124149-14.69664830735$ 1871552475021.56987165875 1885152475021.56987165875 1895512475021.56987165875 190156250514.288272927526 191516250514.288272927526 192165250514.288272927526 193615250514.288272927526 194561250514.288272927526 195651250514.288272927526 196157256523.765508912182 197517256523.765508912182 198175256523.765508912182 199715256523.765508912182 200571256523.765508912182 201751256523.765508912182 $20216626253-5.933605469549$ $20361626253-5.933605469549$ $20466126253-5.933605469549$ $20516726554-4.025216576127$ $20661726554-4.025216576127$ $20717626554-4.025216576127$ $20871626554-4.025216576127$ $20967126554-4.025216576127$ $21076126554-4.025216576127$ 211177271550.5108126371262 212717271550.5108126371262 213771271550.5108126371262 2142232755728.96247263013 2152322755728.96247263013 2163222755728.96247263013 $21722427858-12.37011365171$ $21824227858-12.37011365171$ $21942227858-12.37011365171$ 2202262846020.11311603825 2212622846020.11311603825 2226222846020.11311603825 223227287613.792947921503 224272287613.792947921503 225722287613.792947921503 2262332906272.52130840274 2273232906272.52130840274 2283322906272.52130840274 229236305657.294774912098 230326305657.294774912098 231263305657.294774912098 232623305657.294774912098 233362305657.294774912098 
234632305657.294774912098 $23523731166-4.883544820034$ $23632731166-4.883544820034$ $23727331166-4.883544820034$ $23872331166-4.883544820034$ $23937231166-4.883544820034$ $24073231166-4.883544820034$ 2412443176711.74456028721 2424243176711.74456028721 2434423176711.74456028721 $24424532068-17.62383671238$ $24542532068-17.62383671238$ $24625432068-17.62383671238$ $24752432068-17.62383671238$ $24845232068-17.62383671238$ $24954232068-17.62383671238$ $25024733270-21.23971028644$ $25142733270-21.23971028644$ $25227433270-21.23971028644$ $25372433270-21.23971028644$ $25447233270-21.23971028644$ $25574233270-21.23971028644$ $25625533871-1.805370143935$ $25752533871-1.805370143935$ $25855233871-1.805370143935$ 259256341721.049161620674 260526341721.049161620674 261265341721.049161620674 262625341721.049161620674 263562341721.049161620674 264652341721.049161620674 $26525734773-1.721904721752$ $26652734773-1.721904721752$ $26727534773-1.721904721752$ $26872534773-1.721904721752$ $26957234773-1.721904721752$ $27075234773-1.721904721752$ $27126635374-2.703630583719$ $27262635374-2.703630583719$ $27366235374-2.703630583719$ 274277362762.897148804158 275727362762.897148804158 276772362762.897148804158 2773333657717.42453285258 $27833436678-12.11294555136$ $27934336678-12.11294555136$ $28043336678-12.11294555136$ $28133536979-35.29382150647$ $28235336979-35.29382150647$ $28353336979-35.29382150647$ 2843363728010.38404715967 2853633728010.38404715967 2866333728010.38404715967 $28733737581-17.93629011454$ $28837337581-17.93629011454$ $28973337581-17.93629011454$ $29034538183-22.73038159281$ $29143538183-22.73038159281$ $29235438183-22.73038159281$ $29353438183-22.73038159281$ $29445338183-22.73038159281$ $29554338183-22.73038159281$ 296346387841.300092342892 297436387841.300092342892 298364387841.300092342892 299634387841.300092342892 
300463387841.300092342892 301643387841.300092342892 $30234739385-17.48887154859$ $30343739385-17.48887154859$ $30437439385-17.48887154859$ $30573439385-17.48887154859$ $30647339385-17.48887154859$ $30774339385-17.48887154859$ $30835539986-2.056610308645$ $30953539986-2.056610308645$ $31055339986-2.056610308645$ 311356402878.078886084373 312536402878.078886084373 313365402878.078886084373 314635402878.078886084373 315563402878.078886084373 316653402878.078886084373 $31735740888-0.2482469831631$ $31853740888-0.2482469831631$ $31937540888-0.2482469831631$ $32073540888-0.2482469831631$ $32157340888-0.2482469831631$ $32275340888-0.2482469831631$ $32336641489-2.50173103362$ $32463641489-2.50173103362$ $32566341489-2.50173103362$ $32636741790-0.4342331196048$ $32763741790-0.4342331196048$ $32837641790-0.4342331196048$ $32973641790-0.4342331196048$ $33067341790-0.4342331196048$ $33176341790-0.4342331196048$ $33237742391-1.325449511961$ $33373742391-1.325449511961$ $33477342391-1.325449511961$ 3354444269223.83083483722 $33644542793-5.509846605986$ $33745442793-5.509846605986$ $33854442793-5.509846605986$ $33944743395-13.62372394432$ $34047443395-13.62372394432$ $34174443395-13.62372394432$ 3424554369610.37823598583 3435454369610.37823598583 3445544369610.37823598583 345456439978.377772075017 346546439978.377772075017 347465439978.377772075017 348645439978.377772075017 349564439978.377772075017 350654439978.377772075017 351457445982.030808197897 352547445982.030808197897 353475445982.030808197897 354745445982.030808197897 355574445982.030808197897 356754445982.030808197897 $35746645199-0.0785088240537$ $35864645199-0.0785088240537$ $35966445199-0.0785088240537$ 3604674541001.232550176426 3616474541001.232550176426 3624764541001.232550176426 3637464541001.232550176426 3646744541001.232550176426 3657644541001.232550176426 
3664774601011.894960872626 3677474601011.894960872626 3687744601011.894960872626 36955546310234.20639506883 37055646410313.8552589948 37156546410313.8552589948 37265546410313.8552589948 3735574671048.021134958196 3745754671048.021134958196 3757554671048.021134958196 $376577479107-2.173821730238$ $377757479107-2.173821730238$ $378775479107-2.173821730238$ $379666482108-3.505178111843$ $380667483109-2.999109235101$ $381676483109-2.999109235101$ $382766483109-2.999109235101$ $383677486110-2.287330211179$ $384767486110-2.287330211179$ $385776486110-2.287330211179$ $386777489111-4.737023728189$

TRIPLETTYPE PARAMS:

INDEX: 7 ATOMS: N N O

PAIRS: NN NO NO UNIQUE: -1 TOTAL: 408

index I powers I equiv index I param index I parameter

00110054.93249718806

$101211-53.33874329474$

$202111-53.33874329474$

$301332-7.722341226777$

$403132-7.722341226777$

$501453-8.230464798728$

$604153-8.230464798728$

$701695-14.86621104923$

$806195-14.86621104923$

$9017116-1.448320222883$

$10071116-1.448320222883$

$11022137-10.65622989924$

$12023148-35.57189079905$

$13032148-35.57189079905$ $140251810-4.49704059415$ $150521810-4.49704059415$ 1602620114.786003793321 1706220114.786003793321 $180272212-3.014924001758$ $190722212-3.014924001758$ $200332413-38.0271398729$ $210342514-4.232829223569$ $220432514-4.232829223569$ $230352715-11.67821029954$ $240532715-11.67821029954$ $250362916-5.672181904135$ $260632916-5.672181904135$ $270373117-10.09668286593$ $280733117-10.09668286593$ 29044331819.36705895517 3004534194.842897858192 3105434194.842897858192 3204636207.022272611181 3306436207.022272611181 $340473821-14.76699035052$ $350743821-14.76699035052$ $360554022-5.78325842283$ $370574324-5.371040943477$ $380754324-5.371040943477$ 
3906645253.96994476077 $400674626-2.101739411757$ $410764626-2.101739411757$ 4207748276.091726882356 43101492834.27215498996 44110492834.27215498996 $451025129-16.86500486824$ $461205129-16.86500486824$ $471055732-3.142851375279$ $481505732-3.142851375279$ $491065933-8.600472007218$ $501605933-8.600472007218$ 5110761345.532291438264 5217061345.532291438264 $531116335-89.66111586264$ 54113663716.04133070416 55131663716.04133070416 56114683853.46097427571 57141683853.46097427571 58115703943.89461964307 59151703943.89461964307 $601167240-4.115589578779$ $611617240-4.115589578779$ $621227642-29.43155727634$ $631237743-40.36284724609$ $641327743-40.36284724609$ 65124794410.04839216157 66142794410.04839216157 67126834639.10637201649 68162834639.10637201649 69127854712.98463581317 70172854712.98463581317 $711348849-0.8964919466208$ $721438849-0.8964919466208$ 73135905028.26183788255 74153905028.26183788255 75136925129.54740241047 76163925129.54740241047 77144965311.91090652635 7814597541.12772949049 7915497541.12772949049 80146995531.03559679213 81164995531.03559679213 $8214710156-0.8451047888603$ $8317410156-0.8451047888603$ $8415710659-8.17219729504$ $8517510659-8.17219729504$ 861661086010.62769522854 87167109612.387728954801 88176109612.387728954801 89177111621.715411659062 $9020111263-8.354654863472$ $9121011263-8.354654863472$ $9220211464-5.379633050081$ $9322011464-5.379633050081$ $9420311665-6.903001714421$ $9523011665-6.903001714421$ $9620411866-25.9246132514$ $9724011866-25.9246132514$ $9820512067-21.25591816995$ $9925012067-21.25591816995$ $10020612268-21.02456458543$ $10126012268-21.02456458543$ $10220712469-5.689060351595$ $10327012469-5.689060351595$ $10421112670-0.175690083106$ 
105213129723.989815626768 106231129723.989815626768 $10721513374-4.764375003834$ $10825113374-4.764375003834$ $10921613575-3.69605386985$ $11026113575-3.69605386985$ 111217137769.803535866076 112271137769.803535866076 1132231407813.28531584236 1142321407813.28531584236 $11522414279-23.16922795059$ $11624214279-23.16922795059$ $11722614681-13.03009708444$ $11826214681-13.03009708444$ 119234151845.036238790072 120243151845.036238790072 121235153853.322106350197 122253153853.322106350197 1232361558612.22544383041 1242631558612.22544383041 125237157871.556309142447 126273157871.556309142447 $12724616290-2.185453979847$ $12826416290-2.185453979847$ $12924716491-21.75841513969$ $13027416491-21.75841513969$ 131256167936.501005791451 132265167936.501005791451 133266171951.632866778833 134267172960.03614698768449 135276172960.03614698768449 1362771749713.69410590272 $13730217799-15.55864176905$ $13832017799-15.55864176905$ $139303179100-14.61953449753$ $140330179100-14.61953449753$ $141304181101-24.38191072553$ $142340181101-24.38191072553$ $143305183102-21.43741694172$ $144350183102-21.43741694172$ $145306185103-26.74606486608$ $146360185103-26.74606486608$ $147307187104-2.113323837974$ $148370187104-2.113323837974$ $149311189105-76.93819393174$ 15031219010623.85155321814 15132119010623.85155321814 1523131921076.895686237339 1533311921076.895686237339 1543151961091.454333352192 1553511961091.454333352192 $156316198110-2.986316459327$ $157361198110-2.986316459327$ 1583172001114.678874066294 1593712001114.678874066294 1603232031134.06838041961 1613322031134.06838041961 $162324205114-17.726290594$ $163342205114-17.726290594$ $164325207115-9.903640310064$ $165352207115-9.903640310064$ 1663272111175.566934419607 1673722111175.566934419607 $168334214119-21.10001390521$ $169343214119-21.10001390521$ $170335216120-3.791398918744$ 
$171353216120-3.791398918744$ 17233621812124.08181566838 17336321812124.08181566838 $174344222123-25.46895829866$ $175345223124-22.71004915$ $176354223124-22.71004915$ $177347227126-5.884805228032$ $178374227126-5.884805228032$ $179355229127-21.46943980974$ $180356230128-3.525142893154$ $181365230128-3.525142893154$ $182357232129-9.303495758191$ $183375232129-9.303495758191$ $184366234130-3.060351842998$ 1853672351311.40311094328 1863762351311.40311094328 $187377237132-0.9247875040142$ $188401238133-15.64281097489$ $189410238133-15.64281097489$ $190402240134-6.005743849694$ $191420240134-6.005743849694$ 1924042441365.818740423557 1934402441365.818740423557 1944052461377.859956034973 1954502461377.859956034973 $196406248138-8.922742342284$ $197460248138-8.922742342284$ $198407250139-6.712893386489$ $199470250139-6.712893386489$ 2004112521402.229184758438 $201412253141-9.262417442051$ $202421253141-9.262417442051$ 2034132551423.916540568258 2044312551423.916540568258 20541525914424.66484606667 20645125914424.66484606667 20741626114516.6209466746 20846126114516.6209466746 20941726314613.92194386612 21047126314613.92194386612 21142326614818.61157576011 21243226614818.61157576011 21342426814918.78204632717 21444226814918.78204632717 21542527015024.37715706926 21645227015024.37715706926 $217426272151-7.698548546756$ $218462272151-7.698548546756$ $219427274152-8.224757252557$ $220472274152-8.224757252557$ $221433276153-12.4895329002$ 2224342771541.453697720902 2234432771541.453697720902 22443628115611.45629361074 22546328115611.45629361074 22644428515817.83202025905 $227445286159-4.086307271069$ $228454286159-4.086307271069$ $229446288160-8.257736415818$ $230464288160-8.257736415818$ $231447290161-22.41709453535$ $232474290161-22.41709453535$ $233455292162-7.309284954912$ 2344562931631.317521000198 2354652931631.317521000198 $236457295164-2.534411046068$ 
$237475295164-2.534411046068$ $238466297165-6.011808427291$ $239467298166-2.990055760838$ $240476298166-2.990055760838$ 2414773001678.475709460733 2425013011687.306312514902 2435103011687.306312514902 $244502303169-10.21457774351$ $245520303169-10.21457774351$ $246503305170-5.462711497057$ $247530305170-5.462711497057$ $248504307171-3.855677505203$ $249540307171-3.855677505203$ 2505053091723.161217759949 2515503091723.161217759949 $252506311173-6.516746255376$ $253560311173-6.516746255376$ $254507313174-1.222566832575$ $255570313174-1.222566832575$ 25651231617610.03633470943 25752131617610.03633470943 $258513318177-6.304438965301$ $259531318177-6.304438965301$ $260514320178-7.232127612687$ $261541320178-7.232127612687$ 26251532217917.72041444378 26355132217917.72041444378 26451632418012.41613767795 26556132418012.41613767795 $266517326181-1.218330429971$ $267571326181-1.218330429971$ 2685243311848.911481585826 2695423311848.911481585826 27052533318513.46311945505 27155233318513.46311945505 27252633518610.30365460449 27356233518610.30365460449 $274527337187-0.8466806641847$ $275572337187-0.8466806641847$ $276533339188-27.23015440933$ $277534340189-10.58425383603$ $278543340189-10.58425383603$ 27953634419120.49021129346 28056334419120.49021129346 $281537346192-9.069169068159$ $282573346192-9.069169068159$ 28354434819310.254298472 2845463511958.074779371283 2855643511958.074779371283 $286547353196-5.852557064313$ $287574353196-5.852557064313$ $288555355197-4.044017071458$ 2895563561984.413244264364 2905653561984.413244264364 $291557358199-8.665167777785$ $292575358199-8.665167777785$ 2935663602000.8834195333631 2945673612010.5218095196938 2955763612010.5218095196938 $296577363202-3.785289977082$ $297601364203-12.47370965096$ $298610364203-12.47370965096$ $299602366204-4.717452331318$ $300620366204-4.717452331318$ $301603368205-15.00633676733$ $302630368205-15.00633676733$ 
$303604370206-6.272819195665$ $304640370206-6.272819195665$ $305605372207-2.272622534063$ $306650372207-2.272622534063$ $307606374208-0.8192997286088$ $308660374208-0.8192997286088$ $309607376209-3.614156724651$ $310670376209-3.614156724651$ $311611378210-18.32229745524$ $312613381212-11.3701592534$ $313631381212-11.3701592534$ 31461538521412.65634203494 31565138521412.65634203494 31661638721513.96441756226 31766138721513.96441756226 3186173892161.045707409654 3196713892161.045707409654 $320624394219-1.123726736014$ $321642394219-1.123726736014$ 3226253962208.617975340801 3236523962208.617975340801 3246263982212.653404226734 3256623982212.653404226734 $326627400222-2.443220640673$ $327672400222-2.443220640673$ $328633402223-4.316884469312$ $329634403224-1.166332061602$ $330643403224-1.166332061602$ 3316354052256.869402219231 3326534052256.869402219231 3336364072268.08228760799 3346634072268.08228760799 $335637409227-1.519540441884$ $336673409227-1.519540441884$ 3376454122290.05900817907617 3386544122290.05900817907617 $339646414230-0.08317467795252$ $340664414230-0.08317467795252$ $341647416231-7.588317293464$ $342674416231-7.588317293464$ 3436554182323.380870735045 3446564192332.564203126092 3456654192332.564203126092 $346657421234-1.686935002052$ $347675421234-1.686935002052$ $348666423235-0.2706456726569$ $349667424236-1.009811874317$ $350676424236-1.009811874317$ 3516774262372.077229265786 35270142723810.10821575585 35371042723810.10821575585 3547024292393.951204012012 3557204292393.951204012012 3567034312400.8374523636227 3577304312400.8374523636227 $358704433241-11.14100574987$ $359740433241-11.14100574987$ $360705435242-5.174174326689$ $361750435242-5.174174326689$ $362706437243-5.465086826949$ $363760437243-5.465086826949$ 3647074392441.221633171859 3657704392441.221633171859 36671244224637.62674083265 36772144224637.62674083265 3687134442472.975517312733 
3697314442472.975517312733 $370714446248-7.08632341722$ $371741446248-7.08632341722$ $372715448249-16.79936339379$ $373751448249-16.79936339379$ $374716450250-4.14272530224$ $375761450250-4.14272530224$ $376717452251-9.376271131645$ $377771452251-9.376271131645$ 37872245425224.98620533655 37972345525324.08086396901 38073245525324.08086396901 3817254592555.466773895065 3827524592555.466773895065 3837264612561.866996195613 3847624612561.866996195613 3857274632573.94101272235 3867724632573.94101272235 3877334652585.622336358552 3887344662591.482267401383 3897434662591.482267401383 $390735468260-7.894208475806$ $391753468260-7.894208475806$ 3927364702614.276939175105 3937634702614.276939175105 $394737472262-8.170518049717$ $395773472262-8.170518049717$ 3967444742630.5704125388365 3977464772651.984107940033 3987644772651.984107940033 $399755481267-11.92336042723$ $400756482268-0.8146731004229$ $401765482268-0.8146731004229$ $402757484269-7.799024421501$ $403775484269-7.799024421501$ $404766486270-0.9995661626064$ $405767487271-0.4045932734308$ $406776487271-0.4045932734308$ $407777489272-3.750430113672$

TRIPLETTYPE PARAMS:

INDEX: 8 ATOMS: N O O

PAIRS: NO NO OO UNIQUE: -1 TOTAL: 427 index I powers I equiv index I param index I parameter

0011007.492738192905 1101007.492738192905 $201221-9.705516768803$ $310221-9.705516768803$ $401342-32.85849517602$ $510342-32.85849517602$ $601463-3.852252873846$ $710463-3.852252873846$ $801584-2.620205619208$ $910584-2.620205619208$ 1001610511.18028034599 1110610511.18028034599 $12017126-0.9798774296003$ $13107126-0.9798774296003$ $14021147-5.411238626631$ $15201147-5.411238626631$ $16022168-6.840640297734$ $17202168-6.840640297734$ $18023189-19.82125719278$ $19203189-19.82125719278$

$200242010-0.9046081590572$ 
$212042010-0.9046081590572$ 2202522111.604337901668 2320522111.604337901668 2402624128.854840525384 2520624128.854840525384 2602726136.249162136099 2720726136.249162136099 2803128140.925468513564 2930128140.925468513564 $300323015-8.460868347234$ $313023015-8.460868347234$ $320333216-33.50050393335$ $333033216-33.50050393335$ $340343417-9.21874913991$ $353043417-9.21874913991$ $360353618-11.37233993777$ $373053618-11.37233993777$ 3803638194.76632033691 3930638194.76632033691 40041422135.83291038909 41401422135.83291038909 $420424422-4.698300663951$ $434024422-4.698300663951$ $440434623-14.63142219205$ $454034623-14.63142219205$ 460444824 -18.93896881986 $474044824-18.93896881986$ $480455025-23.31651382562$ $494055025-23.31651382562$ $500465226-6.173826624305$ $514065226-6.173826624305$ $520475427-3.00399187507$ $534075427-3.00399187507$ 5405156284.077599854281 5550156284.077599854281 56052582914.93003603201 57502582914.93003603201 5805462317.375561257778 5950462317.375561257778 $600556432-5.802134239066$ $615056432-5.802134239066$ $620566633-3.249686013614$ $635066633-3.249686013614$ $640576834-0.8837677377082$ $655076834-0.8837677377082$ $660617035-2.042812453018$ $676017035-2.042812453018$ 6806272363.122392588963 6960272363.122392588963 $700637437-0.7011980348981$ $716037437-0.7011980348981$ 7206578390.06158789890719 7360578390.06158789890719 $740668040-2.268672564478$ $756068040-2.268672564478$ 7606782410.174486684943 7760782410.174486684943 $780718442-27.45239795014$ $797018442-27.45239795014$ 8007286436.832934093356 8170286436.832934093356 $820738844-4.304470200019$ $837038844-4.304470200019$ 8407490454.205451986925 8570490454.205451986925 $860769447-2.131145755998$ 
$877069447-2.131145755998$ $880779648-0.1990286268211$ $897079648-0.1990286268211$ $9011210051-35.55420606596$ $9111310152-68.49020010027$ $9211410253-26.87436176845$ 931161045547.60366609284 941171055622.06114457102 $9512010657-66.55504331886$ $9621010657-66.55504331886$ 97121108582.719498478697 98211108582.719498478697 $9912211059-53.5955559916$ $10021211059-53.5955559916$ 101124114614.600469493348 102214114614.600469493348 103125116628.319672647581 104215116628.319672647581 $10512712064-0.8306903862484$ $10621712064-0.8306903862484$ $10713012265-34.77571808346$ $10831012265-34.77571808346$ 1091311246638.60939291863 1103111246638.60939291863 $11113312868-5.126256450855$ $11231312868-5.126256450855$ 1131341306914.66072119825 1143141306914.66072119825 $11513513270-18.48619842636$ $11631513270-18.48619842636$ 1171361347111.91310679583 1183161347111.91310679583 119137136720.1138607591951 120317136720.1138607591951 $12114013873-11.99902414973$ $12241013873-11.99902414973$ 1231411407462.31057588282 1244111407462.31057588282 125142142754.819048435233 126412142754.819048435233 $12714314476-2.608180573879$ $12841314476-2.608180573879$ $12914414677-16.56572833948$ $13041414677-16.56572833948$ $13114514878-42.74921624038$ $13241514878-42.74921624038$ $13314615079-30.00946114204$ $13441615079-30.00946114204$ $13514715280-12.60993052484$ $13641715280-12.60993052484$ $13715015481-5.780811007962$ $13851015481-5.780811007962$ 1391511568254.89059741679 1405111568254.89059741679 1411521588324.95457230815 1425121588324.95457230815 1431531608420.85945018257 1445131608420.85945018257 1451541628519.01683366942 1465141628519.01683366942 $14715516486-2.666275198705$ $14851516486-2.666275198705$ 149156166870.9580234834961 150516166870.9580234834961 $15116017089-12.59632139541$ $15261017089-12.59632139541$ 
$15316117290-22.29141606212$ $15461117290-22.29141606212$ 155165180945.052735301082 156615180945.052735301082 $15716618295-10.29080278335$ $15861618295-10.29080278335$ 159167184966.240259555321 160617184966.240259555321 $16117018697-9.29802588551$ $16271018697-9.29802588551$ $16317118898-39.51377319225$ $16471118898-39.51377319225$ $165173192100-0.02632808540697$ $166713192100-0.02632808540697$ 16717419410110.77980040833 16871419410110.77980040833 16917519610211.98577545405 17071519610211.98577545405 1711772001041.347610004185 1727172001041.347610004185 $173220202105-1.543240326978$ $174222204107-13.32177618993$ $175223205108-12.23671844352$ 17622520711036.89725849159 17722620811124.3967896686 17822720911228.25778777187 $179230210113-26.47562949687$ $180320210113-26.47562949687$ $181231212114-27.38539352955$ $182321212114-27.38539352955$ 1832332161164.334557144934 1843232161164.334557144934 18523421811717.41946545173 18632421811717.41946545173 18723522011814.03027632598 18832522011814.03027632598 1892372241207.515058913695 1903272241207.515058913695 $191240226121-2.183642688455$ $192420226121-2.183642688455$ 19324122812235.34141807563 19442122812235.34141807563 $195243232124-4.509189135316$ $196423232124-4.509189135316$ $197244234125-16.12018556696$ $198424234125-16.12018556696$ $199246238127-1.553448077814$ $200426238127-1.553448077814$ 20124724012811.22740868784 20242724012811.22740868784 $203250242129-18.65668868521$ $204520242129-18.65668868521$ $205251244130-30.7928138978$ $206521244130-30.7928138978$ 20725224613123.80468619417 20852224613123.80468619417 20925324813218.96357960809 21052324813218.96357960809 21125425013322.39831413257 21252425013322.39831413257 2132552521344.594134426579 2145252521344.594134426579 $215256254135-11.62048591948$ $216526254135-11.62048591948$ 2172572561361.399217401858 2185272561361.399217401858 
$219260258137-17.16169212657$ $220620258137-17.16169212657$ $221262262139-15.30878444579$ $222622262139-15.30878444579$ 22326326414015.66197347564 22462326414015.66197347564 $225264266141-8.583055732724$ $226624266141-8.583055732724$ 22726526814213.25364010256 22862526814213.25364010256 $229266270143-6.737326307975$ $230626270143-6.737326307975$ 2312672721443.994719497574 2326272721443.994719497574 $233270274145-8.587770457065$ $234720274145-8.587770457065$ $235271276146-43.31641040581$ $236721276146-43.31641040581$ 2372722781475.503123192528 2387222781475.503123192528 $239273280148-1.066918753786$ $240723280148-1.066918753786$ 2412742821494.639226389685 2427242821494.639226389685 $243275284150-2.587992624725$ $244725284150-2.587992624725$ $245276286151-7.906955284728$ $246726286151-7.906955284728$ $247277288152-2.517438544016$ $248727288152-2.517438544016$ $249330290153-24.20243553259$ $250331291154-2.347576928171$ 25133229215555.34817889414 25233329315632.30221312789 25333429415755.88295685047 $254336296159-1.316171712886$ 2553372971600.8029098483617 2563402981610.7593459323696 2574302981610.7593459323696 $258341300162-8.888765340356$ $259431300162-8.888765340356$ 26034230216325.94969397985 26143230216325.94969397985 $262345308166-4.170881486826$ $263435308166-4.170881486826$ $264346310167-19.97843777807$ $265436310167-19.97843777807$ 2663473121685.167824554795 2674373121685.167824554795 $268350314169-6.952659047412$ $269530314169-6.952659047412$ 27035231817127.52973681298 27153231817127.52973681298 27235332017212.92662378549 27353332017212.92662378549 27435432217312.01782579248 27553432217312.01782579248 $276355324174-0.3673460762276$ $277535324174-0.3673460762276$ $278356326175-12.41447933062$ $279536326175-12.41447933062$ 2803573281761.227827602605 2815373281761.227827602605 $282360330177-12.88942097927$ $283630330177-12.88942097927$ $284361332178-17.82289569951$ 
$285631332178-17.82289569951$ $286362334179-8.691202979237$ $287632334179-8.691202979237$ 2883633361805.900142725934 2896333361805.900142725934 $290364338181-12.80802711968$ $291634338181-12.80802711968$ 2923653401828.583030495077 2936353401828.583030495077 $294366342183-13.63198054417$ $295636342183-13.63198054417$ 2963673441846.873666900973 2976373441846.873666900973 $298370346185-8.013566611857$ $299730346185-8.013566611857$ $300371348186-20.15870758852$ $301731348186-20.15870758852$ $302372350187-0.1704758921859$ $303732350187-0.1704758921859$ 3043733521881.092446197633 3057333521881.092446197633 3063743541892.52129804038 3077343541892.52129804038 3083753561901.876164064299 3097353561901.876164064299 $310376358191-5.762692404996$ $311736358191-5.762692404996$ $312377360192-1.741150250929$ $313737360192-1.741150250929$ 3144403621937.285537192561 31544236419514.59095792677 $316443365196-5.905425045776$ $317444366197-16.21727131476$ 3184453671983.298752501687 $319446368199-10.49058054174$ 3204473692009.74085331832 $321451372202-18.50212963345$ $322541372202-18.50212963345$ 32345237420314.69723598733 32454237420314.69723598733 $325454378205-14.14979697918$ $326544378205-14.14979697918$ $327455380206-7.182430844972$ $328545380206-7.182430844972$ $329456382207-24.04290653249$ $330546382207-24.04290653249$ 3314573842081.58052726498 3325473842081.58052726498 $333460386209-6.845480257974$ $334640386209-6.845480257974$ 33546138821014.51817495842 33664138821014.51817495842 $337462390211-10.60793291631$ $338642390211-10.60793291631$ 33946339221213.23966217783 34064339221213.23966217783 $341464394213-19.29998262493$ $342644394213-19.29998262493$ 3434653962145.723713046573 3446453962145.723713046573 $345466398215-11.84466391367$ $346646398215-11.84466391367$ 3474674002162.385904622879 3486474002162.385904622879 $349470402217-4.980912572287$ $350740402217-4.980912572287$ 
$351471404218-7.834886943246$ $352741404218-7.834886943246$ $353472406219-4.506415525829$ $354742406219-4.506415525829$ $355474410221-11.16032394111$ $356744410221-11.16032394111$ $357475412222-5.724837326613$ $358745412222-5.724837326613$ $359476414223-10.68736828013$ $360746414223-10.68736828013$ $361477416224-1.836239520695$ $362747416224-1.836239520695$ 3635504182253.927183966322 36455141922612.9374085478 $365553421228-8.016842238251$ $366554422229-28.06631335844$ $367555423230-10.86308225631$ $368556424231-19.63241305708$ 3695614282346.619647807591 3706514282346.619647807591 $371562430235-2.58875020652$ $372652430235-2.58875020652$ 3735634322363.774418582062 3746534322363.774418582062 $375564434237-18.14677947302$ $376654434237-18.14677947302$ $377565436238-2.19725238607$ $378655436238-2.19725238607$ $379566438239-13.68749872822$ $380656438239-13.68749872822$ 3815674402401.663419219098 3826574402401.663419219098 3835704422411.414905723078 3847504422411.414905723078 3855714442425.238196615371 3867514442425.238196615371 $387572446243-2.181253206747$ $388752446243-2.181253206747$ $389573448244-1.139419928713$ $390753448244-1.139419928713$ $391574450245-9.482634848148$ $392754450245-9.482634848148$ $393575452246-1.17319177772$ $394755452246-1.17319177772$ $395576454247-6.078305120069$ $396756454247-6.078305120069$ $397577456248-0.09020959227967$ $398757456248-0.09020959227967$ $399660458249-1.359097716759$ 40066145925020.22397537619 $401662460251-5.689119747672$ 40266346125210.94346108862 $403664462253-11.8986442468$ 4046654632541.933303803146 $405666464255-6.740700110758$ 4066674652560.07619000901983 4076714682588.232557079551 4087614682588.232557079551 $409672470259-2.444043353672$ $410762470259-2.444043353672$ 4116734722604.690252472347 4127634722604.690252472347 $413674474261-7.68006474331$ $414764474261-7.68006474331$ $415676478263-5.093868314876$ $416766478263-5.093868314876$ 
4176774802640.4318127568363 4187674802640.4318127568363 4197704822653.048449598901 4207714832664.462280510018 4217724842672.556467764287 4227734852682.29212711411 $423774486269-1.480074532334$ 4247754872701.973353602392 $425776488271-1.262021749745$ 4267774892720.6492179626092

TRIPLETTYPE PARAMS:

INDEX: 9 ATOMS: O O O

PAIRS: OO OO OO UNIQUE: -1 TOTAL: 395

index | powers | equiv index | param index | parameter

00110043.09391346426

11010043.09391346426

21100043.09391346426

$301231-7.158385827808$

$410231-7.158385827808$

$502131-7.158385827808$

$620131-7.158385827808$

$712031-7.158385827808$

$821031-7.158385827808$

901415310.5623244125

1010415310.5623244125

1104115310.5623244125

1240115310.5623244125

1314015310.5623244125

1441015310.5623244125

$15017336-5.429512396655$

$16107336-5.429512396655$

$17071336-5.429512396655$

$18701336-5.429512396655$

$19170336-5.429512396655$

$20710336-5.429512396655$

$21022397-19.05928608525$

$22202397-19.05928608525$

$23220397-19.05928608525$

$24023428-15.7665173346$

$25203428-15.7665173346$

$26032428-15.7665173346$

$27302428-15.7665173346$

$28230428-15.7665173346$

$29320428-15.7665173346$

$30024489-0.6371576569621$

$31204489-0.6371576569621$

$32042489-0.6371576569621$

$33402489-0.6371576569621$

$34240489-0.6371576569621$

$35420489-0.6371576569621$

3602554101.665933563465

3720554101.665933563465

3805254101.665933563465

3950254101.665933563465

4025054101.665933563465

4152054101.665933563465

4202660119.008373570605

4320660119.008373570605

4406260119.008373570605 4560260119.008373570605 4626060119.008373570605 4762060119.008373570605 $480276612-1.750846744366$ $492076612-1.750846744366$ 
$500726612-1.750846744366$ $517026612-1.750846744366$ $522706612-1.750846744366$ $537206612-1.750846744366$ $540337213-14.25472680722$ $553037213-14.25472680722$ $563307213-14.25472680722$ 57034751413.07195625451 58304751413.07195625451 59043751413.07195625451 60403751413.07195625451 61340751413.07195625451 62430751413.07195625451 6303581157.452748484593 6430581157.452748484593 6505381157.452748484593 6650381157.452748484593 6735081157.452748484593 6853081157.452748484593 69036871612.78302190203 70306871612.78302190203 71063871612.78302190203 72603871612.78302190203 73360871612.78302190203 74630871612.78302190203 $750379317-1.820653454819$ $763079317-1.820653454819$ $770739317-1.820653454819$ $787039317-1.820653454819$ $793709317-1.820653454819$ $807309317-1.820653454819$ 8104499185.991060571909 8240499185.991060571909 8344099185.991060571909 84045102199.928678986736 85405102199.928678986736 86054102199.928678986736 87504102199.928678986736 88450102199.928678986736 89540102199.928678986736 90046108207.038302324627 91406108207.038302324627 92064108207.038302324627 93604108207.038302324627 94460108207.038302324627 95640108207.038302324627 96047114212.95830737389 97407114212.95830737389 98074114212.95830737389 99704114212.95830737389 100470114212.95830737389 101740114212.95830737389 102056123234.179043166839 103506123234.179043166839 104065123234.179043166839 105605123234.179043166839 106560123234.179043166839 107650123234.179043166839 $10805712924-4.785105028293$ $10950712924-4.785105028293$ $11007512924-4.785105028293$ $11170512924-4.785105028293$ $11257012924-4.785105028293$ $11375012924-4.785105028293$ 114066135250.1856149100864 115606135250.1856149100864 
116660135250.1856149100864 117067138262.747500763759 118607138262.747500763759 119076138262.747500763759 120706138262.747500763759 121670138262.747500763759 122760138262.747500763759 $12307714427-4.605053185952$ $12470714427-4.605053185952$ $12577014427-4.605053185952$ $12611315130-52.41399711593$ $12713115130-52.41399711593$ $12831115130-52.41399711593$ 129115157322.682701757507 130151157322.682701757507 131511157322.682701757507 132116160337.175485080088 133161160337.175485080088 134611160337.175485080088 $13512316936-0.4411722540158$ $13621316936-0.4411722540158$ $13713216936-0.4411722540158$ $13831216936-0.4411722540158$ $13923116936-0.4411722540158$ $14032116936-0.4411722540158$ 141124175371.007654043956 142214175371.007654043956 143142175371.007654043956 144412175371.007654043956 145241175371.007654043956 146421175371.007654043956 $14712518138-10.81646490759$ $14821518138-10.81646490759$ $14915218138-10.81646490759$ $15051218138-10.81646490759$ $15125118138-10.81646490759$ $15252118138-10.81646490759$ $15312618739-16.68924302006$ $15421618739-16.68924302006$ $15516218739-16.68924302006$ $15661218739-16.68924302006$ $15726118739-16.68924302006$ $15862118739-16.68924302006$ $15913319941-1.759130208264$ $16031319941-1.759130208264$ $16133119941-1.759130208264$ 1621342024212.73916351251 1633142024212.73916351251 1641432024212.73916351251 1654132024212.73916351251 1663412024212.73916351251 1674312024212.73916351251 $16813621444-12.32746321475$ $16931621444-12.32746321475$ $17016321444-12.32746321475$ $17161321444-12.32746321475$ $17236121444-12.32746321475$ $17363121444-12.32746321475$ $17414422646-4.470977330444$ $17541422646-4.470977330444$ $17644122646-4.470977330444$ $17714623548-6.230406107877$ $17841623548-6.230406107877$ $17916423548-6.230406107877$ $18061423548-6.230406107877$ $18146123548-6.230406107877$ 
$18264123548-6.230406107877$ 183147241490.6281054263737 184417241490.6281054263737 185174241490.6281054263737 186714241490.6281054263737 187471241490.6281054263737 188741241490.6281054263737 $18915524750-10.30372039531$ $19051524750-10.30372039531$ $19155124750-10.30372039531$ $19215625051-8.513914701018$ $19351625051-8.513914701018$ $19416525051-8.513914701018$ $19561525051-8.513914701018$ $19656125051-8.513914701018$ $19765125051-8.513914701018$ 198166262534.983007366183 199616262534.983007366183 200661262534.983007366183 $20116726554-6.45593303596$ $20261726554-6.45593303596$ $20317626554-6.45593303596$ $20471626554-6.45593303596$ $20567126554-6.45593303596$ $20676126554-6.45593303596$ 207177271555.383210098116 208717271555.383210098116 209771271555.383210098116 $21022227456-96.80382992723$ $21122327557-37.8907410136$ $21223227557-37.8907410136$ $21332227557-37.8907410136$ $21422427858-26.69186276599$ $21524227858-26.69186276599$ $21642227858-26.69186276599$ 217225281596.570671205305 218252281596.570671205305 219522281596.570671205305 2202262846019.31056466986 2212622846019.31056466986 2226222846019.31056466986 $22322728761-9.270178229481$ $22427228761-9.270178229481$ $22572228761-9.270178229481$ $22623429363-5.883178897262$ $22732429363-5.883178897262$ $22824329363-5.883178897262$ $22942329363-5.883178897262$ $23034229363-5.883178897262$ $23143229363-5.883178897262$ 232235299646.280650935823 233325299646.280650935823 234253299646.280650935823 235523299646.280650935823 236352299646.280650935823 237532299646.280650935823 238236305652.853482125292 239326305652.853482125292 240263305652.853482125292 241623305652.853482125292 242362305652.853482125292 243632305652.853482125292 244245320685.65964304171 245425320685.65964304171 246254320685.65964304171 247524320685.65964304171 
248452320685.65964304171 249542320685.65964304171 2502463266913.39737236871 2514263266913.39737236871 2522643266913.39737236871 2536243266913.39737236871 2544623266913.39737236871 2556423266913.39737236871 $25624733270-3.804361748725$ $25742733270-3.804361748725$ $25827433270-3.804361748725$ $25972433270-3.804361748725$ $26047233270-3.804361748725$ $26174233270-3.804361748725$ $26225533871-13.31779408406$ $26352533871-13.31779408406$ $26455233871-13.31779408406$ $26525734773-8.052544369465$ $26652734773-8.052544369465$ $26727534773-8.052544369465$ $26872534773-8.052544369465$ $26957234773-8.052544369465$ $27075234773-8.052544369465$ 271266353741.048657089651 272626353741.048657089651 273662353741.048657089651 274267356752.91895781901 275627356752.91895781901 276276356752.91895781901 277726356752.91895781901 278672356752.91895781901 279762356752.91895781901 $28027736276-5.780252673397$ $28172736276-5.780252673397$ $28277236276-5.780252673397$ 2833333657761.61684003502 2843343667815.01352313785 2853433667815.01352313785 2864333667815.01352313785 2873353697910.71383980339 2883533697910.71383980339 2895333697910.71383980339 $29033637280-15.20426797573$ $29136337280-15.20426797573$ $29263337280-15.20426797573$ 293337375810.4764828856617 294373375810.4764828856617 295733375810.4764828856617 $29634437882-3.794304329289$ $29743437882-3.794304329289$ $29844337882-3.794304329289$ 299345381836.812198144588 300435381836.812198144588 301354381836.812198144588 302534381836.812198144588 303453381836.812198144588 304543381836.812198144588 305346387841.69677218769 306436387841.69677218769 307364387841.69677218769 308634387841.69677218769 309463387841.69677218769 310643387841.69677218769 $31134739385-0.3966074018607$ $31243739385-0.3966074018607$ $31337439385-0.3966074018607$ 
$31473439385-0.3966074018607$ $31547339385-0.3966074018607$ $31674339385-0.3966074018607$ $31735539986-6.548362012803$ $31853539986-6.548362012803$ $31955339986-6.548362012803$ $32035640287-3.740055691744$ $32153640287-3.740055691744$ $32236540287-3.740055691744$ $32363540287-3.740055691744$ $32456340287-3.740055691744$ $32565340287-3.740055691744$ 326366414895.278900096408 327636414895.278900096408 328663414895.278900096408 $32936741790-3.088773311459$ $33063741790-3.088773311459$ $33137641790-3.088773311459$ $33273641790-3.088773311459$ $33367341790-3.088773311459$ $33476341790-3.088773311459$ 335377423914.076974776106 336737423914.076974776106 337773423914.076974776106 338444426923.779911310495 $33944542793-2.368009034171$ $34045442793-2.368009034171$ $34154442793-2.368009034171$ 342446430946.008392605741 343464430946.008392605741 344644430946.008392605741 $34544743395-3.387367457377$ $34647443395-3.387367457377$ $34774443395-3.387367457377$ $34845543696-6.600435416966$ $34954543696-6.600435416966$ $35055443696-6.600435416966$ $35145643997-1.931392232957$ $35254643997-1.931392232957$ $35346543997-1.931392232957$ $35464543997-1.931392232957$ $35556443997-1.931392232957$ $35665443997-1.931392232957$ $35745744598-3.899922084967$ $35854744598-3.899922084967$ $35947544598-3.899922084967$ $36074544598-3.899922084967$ $36157444598-3.899922084967$ $36275444598-3.899922084967$ 3634674541002.019261946071 3646474541002.019261946071 3654764541002.019261946071 3667464541002.019261946071 3676744541002.019261946071 3687644541002.019261946071 $369477460101-2.815119684128$ $370747460101-2.815119684128$ $371774460101-2.815119684128$ 3725574671042.530512677691 3735754671042.530512677691 3747554671042.530512677691 3755664701050.7357807844039 3766564701050.7357807844039 3776654701050.7357807844039 $378567473106-1.076453610566$ $379657473106-1.076453610566$ 
$380576473106-1.076453610566$

$381756473106-1.076453610566$

$382675473106-1.076453610566$

$383765473106-1.076453610566$

3845774791071.882389942106

3857574791071.882389942106

3867754791071.882389942106

$387666482108-0.5337095928529$

3886674831091.404519403587

3896764831091.404519403587

3907664831091.404519403587

$391677486110-1.574329601324$

$392767486110-1.574329601324$

$393776486110-1.574329601324$

$394777489111-0.2005911279024$

QUADRUPLET CHEBYSHEV PARAMS

PAIRMAPS: 9

$0 \mathrm{CC}$

$3 \mathrm{CN}$

$4 \mathrm{CO}$

$3 \mathrm{NC}$

$1 \mathrm{NN}$

$5 \mathrm{NO}$

$4 \mathrm{OC}$

$5 \mathrm{ON}$

200

TRIPMAPS: 27

0 CCCCCC

$1 \mathrm{CCCNCN}$

$2 \mathrm{CCCOCO}$

$1 \mathrm{CNCCCN}$

1 CNCNCC

3 CNCNNN

4 CNCONO

3 CNNNCN

4 CNNOCO

$2 \mathrm{COCCCO}$

4 COCNNO

2 COCOCC

$5 \mathrm{COCOOO}$

4 CONOCN

5 COOOCO

3 NNCNCN

6 NNNNNN

7 NNNONO

4 NOCNCO

$4 \mathrm{NOCOCN}$

7 NONNNO

7 NONONN

8 NONOOO

8 NOOONO

5 OOCOCO

8 OONONO

9000000

NO ENERGY OFFSETS: 3

ENERGY OFFSET 10.0

ENERGY OFFSET 2 3908.19887304

ENERGY OFFSET 30.0

ENDFILE 